\title{
Creature forcing and five cardinal characteristics in Cichoń's diagram
}

\author{
Arthur Fischer ${ }^{1}$ - Martin Goldstern ${ }^{2}$. \\ Jakob Kellner ${ }^{2}$ - Saharon Shelah ${ }^{3,4}$
}

Received: 15 January 2014 / Accepted: 22 May 2015 / Published online: 3 June 2017 (C) The Author(s) 2017. This article is an open access publication

Abstract We use a (countable support) creature construction to show that consistently

$$
\mathfrak{d}=\aleph_{1}=\operatorname{cov}(\mathcal{N})<\operatorname{non}(\mathcal{M})<\operatorname{non}(\mathcal{N})<\operatorname{cof}(\mathcal{N})<2^{\aleph_{0}}
$$

The same method shows the consistency of

$$
\mathfrak{d}=\aleph_{1}=\operatorname{cov}(\mathcal{N})<\operatorname{non}(\mathcal{N})<\operatorname{non}(\mathcal{M})<\operatorname{cof}(\mathcal{N})<2^{\aleph_{0}}
$$

Dedicated to the memory of James E. Baumgartner (1943-2011).

We gratefully acknowledge the following partial support: Austrian Science Fund FWF P23875-N13 (first author), P24725-N25 (second author) and I1272-N25 (third author); US National Science Foundation NSF DMS-1362974 (second author), and European Research Council grant ERC-2013-ADG 338821 (fourth author). This is publication 1044 of the fourth author.

Jakob Kellner

jakob.kellner@tuwien.ac.at

http://dmg.tuwien.ac.at/kellner/

Arthur Fischer

arthur.james.fischer@univie.ac.at

Martin Goldstern

martin.goldstern@tuwien.ac.at

http://www.tuwien.ac.at/goldstern/

Saharon Shelah

shelah@math.huji.ac.il

http://shelah.logic.at/

1 Kurt Gödel Research Center for Mathematical Logic, Universität Wien, Währinger Straße 25, 1090 Vienna, Austria

2 Institut für Diskrete Mathematik und Geometrie, Technische Universität Wien, Wiedner Hauptstraße 8-10/104, 1040 Vienna, Austria 
Keywords Set theory of the reals · Creature forcing · Cichon's diagram

Mathematics Subject Classification 03E17 · 03E35 - 03E40

\section{Introduction}

\subsection{The result and its history}

Let $\mathcal{N}$ denote the ideal of Lebesgue null sets, and $\mathcal{M}$ the ideal of meager sets. We prove (see Theorem 6.2.1) that consistently, several cardinal characteristics of Cichon's Diagram (see Fig. 1) are (simultaneously) different:

$$
\aleph_{1}=\operatorname{cov}(\mathcal{N})=\mathfrak{d}<\operatorname{non}(\mathcal{M})<\operatorname{non}(\mathcal{N})<\operatorname{cof}(\mathcal{N})<2^{\aleph_{0}} .
$$

Since our model will satisfy $\mathfrak{d}=\aleph_{1}$, will also have $\operatorname{non}(\mathcal{M})=\operatorname{cof}(\mathcal{M})$. The desired values of the cardinals $\operatorname{non}(\mathcal{M}), \operatorname{non}(\mathcal{N}), \operatorname{cof}(\mathcal{N}), 2^{\aleph_{0}}$ can be chosen quite arbitrarily, as long as they are ordered as indicated and each satisfies $\kappa^{\aleph_{0}}=\kappa$.

A (by now) classical series of theorems [1,4,7-10,14-18] proves these (in)equalities in ZFC and shows that they are the only ones provable. More precisely, all assignments of the values $\aleph_{1}$ and $\aleph_{2}$ to the characteristics in Cichoń's Diagram are consistent, provided they do not contradict the above (in)equalities. (A complete proof can be found in [3, chapter 7].)

This does not answer the question whether three (or more) characteristics can be made simultaneously different. The general expectation is that this should always be possible, but may require quite complicated forcing methods. We cannot use the two best understood methods, countable support iterations of proper forcings (as it forces $2^{\aleph_{0}} \leq \aleph_{2}$ ) and, at least for the "right hand side" of the diagram, we cannot use finite support iterations of ccc forcings in the straightforward way (as it adds lots of Cohen reals, and thus increases $\operatorname{cov}(\mathcal{M})$ to $\left.2^{\aleph_{0}}\right)$.

There are ways to overcome this obstacle. One way would be to first increase the continuum in a "long" finite support iteration, resulting in $\operatorname{cov}(\mathcal{M})=2^{\aleph_{0}}$, and then "collapsing" $\operatorname{cov}(\mathcal{M})$ in another "short" finite support iteration. In a much more sophisticated version of this idea, Mejía [13] recently constructed several models with many simultaneously different cardinal characteristics in Cichoń's Diagram (building on work of Brendle [5], Blass-Shelah [6] and Brendle-Fischer [2]).

We take a different approach, completely avoiding finite support, and use something in between a countable and finite support product (or, a form of iteration with very "restricted memory").

This construction avoids Cohen reals, it is in fact $\omega^{\omega}$-bounding, resulting in $\mathfrak{d}=\aleph_{1}$. This way we get an independence result "orthogonal" to the ccc/finite-support results of Mejía.

The fact that our construction is $\omega^{\omega}$-bounding is not incidental, but rather a necessary consequence of the two features which, in our construction, are needed to guarantee

\footnotetext{
${ }^{3}$ Einstein Institute of Mathematics, Edmond J. Safra Campus, Givat Ram, The Hebrew University of Jerusalem, 91904 Jerusalem, Israel

${ }^{4}$ Department of Mathematics, Rutgers University, New Brunswick, NJ 08854, USA
} 


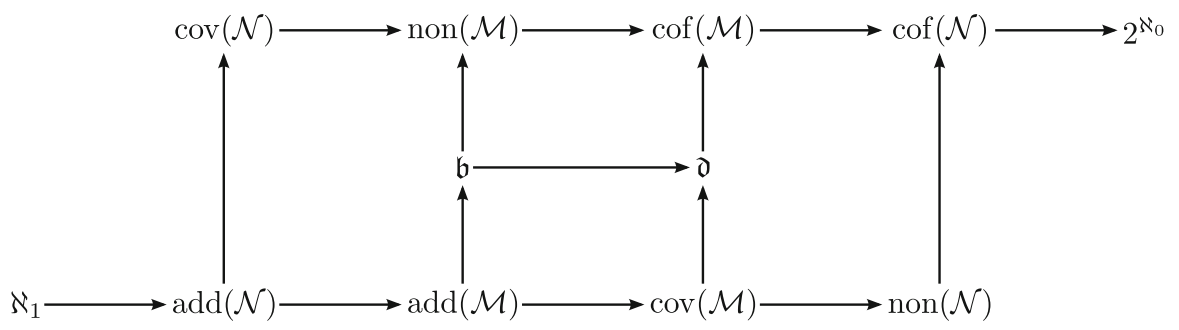

Fig. 1 Cichoń's diagram. An arrow between $\mathfrak{x}$ and $\mathfrak{y}$ indicates that $\mathfrak{x} \leq \mathfrak{y}$. Moreover, $\max (\mathfrak{d}, \operatorname{non}(\mathcal{M}))=$ $\operatorname{cof}(\mathcal{M})$ and $\min (\mathfrak{b}, \operatorname{cov}(\mathcal{M}))=\operatorname{add}(\mathcal{M})$

properness: a "compact" or "finite splitting" version of pure decision, and fusion (which together give a strong version of Baumgartner's Axiom A and in particular properness and $\omega^{\omega}$-bounding).

We think that our construction can be used for various other independence results with $\mathfrak{d}=\aleph_{1}$, but the construction would require considerable remodeling if we want to use it for similar results with $\mathfrak{d}>\aleph_{1}$, even more so for $\mathfrak{b}>\aleph_{1}$.

\subsection{A very informal overview of the construction}

The obvious attempt to prove the theorem would be to find a forcing for each cardinal characteristic $\mathfrak{x}$ that increases $\mathfrak{x}$ but leaves the other characteristics unchanged. More specifically, find the following forcing notions.

- $\mathbb{Q}_{n m}$, adding a new meager set which will contain all old reals. Adding many such sets will tend to make non $(\mathcal{M})$ large.

- $\mathbb{Q}_{n n}$, adding a new measure zero set which will contain all old reals. Adding many such sets will tend to make non $(\mathcal{N})$ large.

- $\mathbb{Q}_{\mathrm{cn}}$, adding a new measure zero set which is not contained in any old measure zero set.

Adding many such sets will tend to make $\operatorname{cof}(\mathcal{N})$ large.

- $\mathbb{Q}_{s k}$, adding a kind of Sacks real, in the sense that the generic real does not change any other cardinal characteristic; in particular, every new real is bounded by an old real, is contained in an old measure zero set, etc.

Adding many such reals will tend to make the continuum large.

For each $t \in\{\mathrm{nm}, \mathrm{nn}, \mathrm{cn}, \mathrm{sk}\}$, our $\mathbb{Q}_{t}$ will be a finitely splitting tree forcing; $\mathbb{Q}_{\mathrm{nm}}$ will be "lim-inf" (think of a tree forcing where we require large splitting at every node, not just infinitely many along every branch; i.e., more like Laver or Cohen than Miller or Sacks; however note that in contrast to Laver all our forcings are finitely splitting); the other ones will be "lim-sup" (think of forcings like Sacks or Silver).

We then fix for each $t$ a cardinal $\kappa_{t}$, and take some kind of product (or, iteration) of $\kappa_{t}$ many copies of $\mathbb{Q}_{t}$, and hope for the best. Here we arrive at the obvious problem: which product or iteration will work? As mentioned above, neither a finite support iteration ${ }^{1}$ nor a countable support iteration will work, and it is not clear why a product

\footnotetext{
${ }^{1}$ To avoid giving a wrong impression, our specific forcings $\mathbb{Q}_{t}$ will not be ccc, so a finite support iteration would not work anyway.
} 
will not collapse the continuum. So we will introduce a modification of the product construction.

The paper is divided into two parts. In part 1 we describe the "general" forcing construction (let us call it the "framework"), in part 2, the "application", we use the framework to construct a specific forcing that proves the main theorem.

Part 1: In Sects. 2-5 we present the "framework". Starting with building blocks (socalled "subatoms"), we define the forcing $\mathbb{Q}$. This is an instance of creature forcing. (The standard reference for creature forcing is Rosłanowski and Shelah [17], but our presentation will be self-contained. Our framework is a continuation of [11,12], where the central requirement to get properness was "decisiveness". In this paper, decisiveness does not appear explicitly, but is implicit in the way that the subatoms are combined to form so-called atoms.)

We fix a set $\Xi$ of indices. (For the application, we will partition $\Xi$ into sets $\Xi_{t}$ of size $\kappa_{t}$ for $t \in\{\mathrm{nm}, \mathrm{nn}, \mathrm{cn}, \mathrm{sk}\}$ as above.) The forcing $\mathbb{Q}$ will "live" on the product $\Xi \times \omega$, i.e., a condition $p \in \mathbb{Q}$ will contain for certain $(\xi, n)$ a "creature" $p(\xi, n)$, a finite object that gives some information about the generic filter.

More specifically, there is a countable subset $\operatorname{supp}(p) \subseteq \Xi$, and for each $\xi \in$ $\operatorname{supp}(p)$ the condition up to some level $n_{0}(\xi)$ consists of a so-called trunk (where a finite initial segment of the generic real $y_{\xi}$ is already completely determined), and for all $n>n_{0}(\xi)$ there is a creature $p(\xi, n)$, an element of a fixed finite set $\mathrm{K}_{\xi, n}$, which gives several (finitely many) possibilities for the corresponding segment of the generic real $y_{\xi}$. We assign a "norm" to the creature, a real number that measures the "number of possibilities" (or, the amount of freedom that the creature leaves for the generic). More possibilities means larger norm.

Moreover, for each $m$ there are only finitely many $\xi$ with $n_{0}(\xi) \leq m$ (i.e., at each level $m$ there are only finitely many creatures of $p$ ). We can then set the norm of $p$ at $m$ to be the minimum of the norms of $p(\xi, n)$ over all $\xi$ "active" at level $m$.

A requirement for a $p$ to be a valid condition in $\mathbb{Q}$ is that the norms at level $m$ diverge to infinity for $m \rightarrow \infty$ (i.e., the lim-inf of the norms is infinite).

So far, $\mathbb{Q}$ seems to be a lim-inf forcing, but recall that we want to use lim-inf as well as lim-sup.

So let us redefine $\mathbb{Q}$. We will "cheat" by allowing "gluing". We declare a subset of $\Xi$ to be the set $\Xi_{1 \mathrm{~s}}$ of "lim-sup indices" (in the application this will be $\Xi_{\mathrm{nn}} \cup \Xi_{\mathrm{cn}}$ ). Forget the "norm of $p$ at level $m$ " and the lim-inf condition above. Instead, we partition the set of levels $\omega$ into finite intervals $\omega=I_{0} \cup I_{1} \cup \ldots$ (this partition depends on the condition and can be coarsened when we go to a stronger condition). For such an interval $I$, we declare all creatures whose levels belong to $I$ to constitute a "compound creature" with a "compound norm", intuitively computed as follows:

- for each $\xi \in \Xi_{1 \mathrm{~s}}$ we set nor $(p, I, \xi)$ to be the maximum of the norms of $p(\xi, m)$ with $m \in I$;

- for other $\xi$ we take the minimum rather than the maximum; and

- we set nor $(p, I)$ to be the minimum of nor $(p, I, \xi)$ for all (finitely many) $\xi$ active at some level in $I$.

The new lim-inf condition is that nor $\left(p, I_{k}\right)$ diverges to infinity with $k \rightarrow \infty$. 
While this may give some basic idea about the construction, things really are more complicated. We will require the well-known "halving" property of creature forcing (to prove Axiom A). Moreover, the Sacks part, i.e., $\mathbb{Q}_{\mathrm{sk}}$ on the indices $\Xi_{\mathrm{Sk}} \subset \Xi$, does not fit well into the framework as presented above and requires special treatment. This will not be very complicated mathematically but will unfortunately make our notation much more awkward and unpleasant.

A central requirement on our building blocks (subatoms) will be another wellknown property of creature-forcing: "bigness". This is a kind of Ramsey property connected to the statement that creatures at a level $m$ are "much bigger" than everything that "happened below $m$ ".

Using these requirements, we will show the following.

- (Assuming $\mathrm{CH}$ in $V$ ) $\mathbb{Q}$ is $\aleph_{2}$-cc. (Accomplished via a standard $\Delta$-system argument.)

- We say that $p$ "essentially decides" a name $\underset{\sim}{\tau}$ of an ordinal if there is a level $m$ such that whenever we increase the trunk of $p$ up to $m$ (for this there are only finitely many possibilities), we know the value of $\underset{\sim}{\tau}$. In other words, knowing the generic up to $m$ (on some finite set of indices), we also know the value of $\underset{\sim}{\tau}$.

- Pure decision and fusion. Given a name $\underset{\sim}{\tau}$ of an ordinal and a condition $p$, we can strengthen $p$ to a condition $q$ essentially deciding $\underset{\sim}{\tau}$. Moreover, we can do this in such a way that $p$ and $q$ agree below a given level $h$ and the norms above this level do not drop below a given bound. (This is called "pure decision".)

This in turn implies "fusion" in that we can iterate this strengthening for infinitely many names $\tau_{\mathcal{L}}$, resulting in a common extension $q_{\infty}$ which essentially decides each $\underset{\sim}{\tau} \ell_{\ell}$.

(While fusion is an obvious property of the framework, pure decision is the central result of part 1, and will use the requirements on bigness and halving).

- The usual standard argument then gives continuous reading (every real is a continuous image of (countably many) generic reals), a strong version of Axiom A, and thus $\omega^{\omega}$-bounding and properness. (Recall that we have "finite splitting", i.e., essentially deciding implies that there are only finitely many potential values.)

- We also get a Lipschitz variant of continuous reading, "rapid reading", which implies that the forcing adds no random reals (and which will be essential for many of the proofs in part 2).

Part 2: In Sections 6-10 we define the specific forcings $\mathbb{Q}_{t}$ (or rather, the building blocks, i.e., the subatoms, for these forcings) for $t \in\{\mathrm{nm}, \mathrm{nn}, \mathrm{cn}\}$ (the Sacks case is already dealt with in part 1).

We prove that these subatoms satisfy the bigness requirements of the framework, and we prove the various parts of the main theorem.

\section{Annotated Contents}

Part 1: We present a forcing framework.

Section 2, p. 1050 Starting with building blocks (the so-called subatomic families, which are black boxes that will be described later) we describe how to build a forcing $\mathbb{Q}$. 
Section 3, p. 1062 We give some simple properties of $\mathbb{Q}$, including the $\aleph_{2}$-cc.

Section 4, p. 1069 We impose additional requirements on the subatomic families, and give an inductive construction that shows how we can choose suitable subatomic families so that the requirements are satisfied.

Section 5, p. 1071 Using the additional requirements, we show that $\mathbb{Q}$ satisfies Axiom $\mathrm{A}$, is $\omega^{\omega}$-bounding and has continuous and rapid reading. This implies $\mathfrak{d}=\operatorname{cov}(\mathcal{N})=\aleph_{1}$ in the generic extension.

Part 2: We give the application.

Section 6, p. 1080 We present the specific forcing. There are four "types" $t, \mathrm{~nm}, \mathrm{nn}$, $\mathrm{cn}$, and sk, corresponding to $\operatorname{non}(\mathcal{M}), \operatorname{non}(\mathcal{N}), \operatorname{cof}(\mathcal{N})$ and the continuum, respectively. The nm-part will be lim-inf, $\mathrm{nn}$ and $\mathrm{cn}$ lim-sup (and sk lim-sup as well, but treated differently). The actual definitions of the $t$-subatoms (other than Sacks) will be given in Sects. 7, 8 and 10. For each type $t$ the forcing will contain a " $t$ part" of size $\kappa_{t}$.

We formulate the main theorem: $\mathbb{Q}$ will force each invariant to be the respective $\kappa_{t}$.

We show that the Sacks part satisfies a Sacks property, which implies $\operatorname{cof}(\mathcal{N}) \leq \kappa_{\mathrm{Cn}}$ in the generic extension.

Using the fact that only the nm-indices are "lim-inf", we show that $\operatorname{non}(\mathcal{M}) \leq \kappa_{\mathrm{nm}}$.

Section 7, p. 1087 We define the nm-subatoms and prove non $(\mathcal{M}) \geq \kappa_{\mathrm{nm}}$.

Section 8, p. 1089 We define the nn-subatoms and prove non $(\mathcal{N}) \geq \kappa_{\mathrm{nn}}$.

Section 9, p. 1091 We mention some simple facts about counting, and use them to define the counting norm, lognor, for the cn subatoms.

Section 10, p. 1094 We define the cn-subatoms and prove $\operatorname{cof}(\mathcal{N}) \geq \kappa_{\mathrm{cn}}$. And finally, we show $\operatorname{non}(\mathcal{N}) \leq \kappa_{\mathrm{nn}}$.

\section{The definition of the forcing $\mathbb{Q}$}

\subsection{Subatomic creatures}

Definition 2.1.1 Let POSS be a finite set. A subatomic family living on POSS consists of a finite set $\mathrm{K}$ (whose elements are called subatomic creatures, or subatoms, for short), a quasiorder $\leq$ on $\mathrm{K}$ and functions poss and nor with domain $\mathrm{K}$, satisfying the following for all $x \in \mathrm{K}$ :

- $\operatorname{poss}(x)$ is a nonempty subset of POSS;

- $\operatorname{nor}(x)$ is a nonnegative real number; and

- $y \leq x$ implies $\operatorname{poss}(y) \subseteq \operatorname{poss}(x)$.

To simplify notation, we further assume:

- if $|\operatorname{poss}(y)|=1$, then nor $(y)<1$; and

- for each $x \in \mathrm{K}$ and $a \in \operatorname{poss}(x)$ there is a $y \leq x$ with $\operatorname{poss}(y)=\{a\}$. (Such a subatom will be called a singleton.) 
Notation 2.1.2 Abusing notation, we will just write $\mathrm{K}$ for the subatomic family ( $\mathrm{K}, \leq$, nor, poss). If $y \leq x$ we will also say that $y$ is "stronger than $x$ " or is "a successor of $x$ ".

Remark 2.1.3 Our subatomic families will also have the following properties (which might make the picture clearer, but will not be used in any proof).

- Each subatom $x$ is determined ${ }^{2}$ by poss $(x)$ (i.e., the function poss $: \mathrm{K} \rightarrow 2^{\text {POSS }}$ is injective). In particular nor $(x)$ is determined by poss $(x)$.

- $\operatorname{poss}(y) \subseteq \operatorname{poss}(x)$ implies nor $(y) \leq \operatorname{nor}(x)$.

- $y \leq x$ iff $\operatorname{poss}(y) \subseteq \operatorname{poss}(x)$.

In the usual way we often identify a natural number $n$ with the set $\{0, \ldots, n-1\}$, and write $m \in n$ for $m<n$; for example in the following definition.

Definition 2.1.4 Fix a natural number $B>0$. We say that a subatom $x \in \mathrm{K}$ has $B$ bigness if for each coloring $c: \operatorname{poss}(x) \rightarrow B$ there is a $y \leq x$ such that $c\lceil\operatorname{poss}(y)$ is constant and nor $(y) \geq \operatorname{nor}(x)-1 .{ }^{3}$ We say that the subatomic family $\mathrm{K}$ has $B$-bigness if each $x \in \mathrm{K}$ has $B$-bigness.

Given a subatom $x$ in a fixed subatomic family $\mathrm{K}$, we have the following facts.

- If $\operatorname{nor}(x) \leq 1$, then $x$ has $B$-bigness for all $B>0$. (Any coloring $c: \operatorname{poss}(x) \rightarrow B$ will be constant on $\operatorname{poss}(y)$ for any singleton $y \leq x$.)

- If $\operatorname{nor}(x) \geq 2$, then $x$ cannot have $|\operatorname{poss}(x)|$-bigness. (The identity function $c$ : $\operatorname{poss}(x) \rightarrow \operatorname{poss}(x)$ is only constant on singleton sets, and any singleton subatom has norm $<\operatorname{nor}(x)-1$.)

- If $x$ has $B$-bigness, then $x$ has $B^{\prime}$-bigness for all $1 \leq B^{\prime} \leq B$.

Example 2.1.5 The basic example of a subatomic family with $B$-bigness is the following "counting norm". For a fixed finite set POSS, a subatom $x$ is a nonempty subset of POSS, with $\operatorname{poss}(x):=x, y \leq x$ defined as $y \subseteq x$, and

$$
\operatorname{nor}(x):=\log _{B}|x| \text {. }
$$

We get a stronger variant of bigness if we divide the norm by $B$ :

$$
\operatorname{nor}^{\prime}(x):=\frac{\log _{B}(|x|)}{B}
$$

Then for each $F: \operatorname{poss}(x) \rightarrow B$ there is a $y \leq x$ such that $F \uparrow \operatorname{poss}(y)$ is constant and $\operatorname{nor}^{\prime}(y) \geq \operatorname{nor}^{\prime}(x)-1 / B$.

\footnotetext{
2 The analogous statement will not be true for "compound creatures" (cf. Definition 2.5.1) because of the halving parameters.

3 As only the number of "colors" is of importance, we may consider the codomain of the coloring function to be any set of cardinality $B$.
} 
Remark 2.1.6 The above example (in the version nor') is actually used for the $\operatorname{non}(\mathcal{M})$-subatoms (cf. 7.1.1). The $\operatorname{cof}(\mathcal{N})$-subatoms (cf. Sect. 10.1) still use a counting norm, i.e., nor $(x)$ only depends on the cardinality of $\operatorname{poss}(x)$, but the relation between $|\operatorname{poss}(x)|$ and nor $(x)$ is more complicated. The non $(\mathcal{N})$-subatoms (cf. Sect. 8.1) will use a different kind of norm which does not just depend on the cardinality of $\operatorname{poss}(x)$, but also on its structure.

Given a subatomic family with 2-bigness, it is straightforward to construct another subatomic family with arbitrary bigness by only altering the norm.

Lemma 2.1.7 If $K$ is a subatomic family with 2-bigness, then given any $b \geq 1$ replacing the norm of $K$ with nor' defined by $\operatorname{nor}^{\prime}(x):=\operatorname{nor}(x) / b$ results in a subatomic family with $2^{b}$-bigness.

Proof Given $x \in \mathrm{K}$, and a coloring $c: \operatorname{poss}(x) \rightarrow \mathcal{P}(b)$, use the 2-bigness of the original subatomic family to inductively pick $x=x_{0} \geq x_{1} \geq \cdots \geq x_{b}=y$ so that for each $i<b$ we have $\operatorname{nor}\left(x_{i+1}\right) \geq \operatorname{nor}\left(x_{i}\right)-1$ and $c_{i}\left\lceil\operatorname{poss}\left(x_{i+1}\right)\right.$ is constant, where $c_{i}: \operatorname{poss}\left(x_{i}\right) \rightarrow 2$ is defined by $c_{i}(a)=1$ iff $i \in c(a)$. Then $c \uparrow \operatorname{poss}(y)$ is constant, and $\operatorname{nor}^{\prime}(y)=\operatorname{nor}(y) / b \geq(\operatorname{nor}(x)-b) / b=\operatorname{nor}^{\prime}(x)-1$.

Remark 2.1.8 Of course, any subatomic family K can be made to have arbitrary bigness by simply ensuring that all subatoms have norm $\leq 1$. The benefit of the method presented in Lemma 2.1.7 is that the norm of each subatom decreases proportionally to the logarithm of the desired bigness. As our construction depends on the existence of subatomic families with "big" bigness and also having subatoms with "large" norm, the above Lemma gives an indication of how this can be achieved.

\subsection{Atomic creatures}

We now describe how to combine subatomic families to create so-called atoms. Fix a natural number $J>0$, and fix a parameter $\ell \in \omega$. We will first define the "measure" of subsets of $J$ with respect to this parameter.

Definition 2.2.1 For $A \subseteq J$, we set

$$
\mu^{\ell}(A):=\frac{\log _{3}(|A|)}{\ell+1}
$$

(or 0 , if $A=\emptyset)^{4}$

We will later use the following easy observation about the "measure".

Lemma 2.2.2 Suppose $k \leq \ell$, and $A_{0}, \ldots, A_{k}$ are subsets of $J$. Then there are pairwise disjoint sets $B_{0}, \ldots, B_{k}$ such that $B_{i} \subseteq A_{i}$, and $\mu^{\ell}\left(B_{i}\right) \geq \mu^{\ell}\left(A_{i}\right)-1$ for all $i \leq k$.

\footnotetext{
${ }^{4}$ So, technically $\mu^{\ell}(A)$ is defined to be $\log _{3}(\max \{|A|, 1\}) /(\ell+1)$.
} 
Proof Note that if for some $i \leq k$ we have that $\mu^{\ell}\left(A_{i}\right) \leq 1$, then simply picking $B_{i}:=$ $\emptyset$ will introduce no obstructions. We may then assume that $\mu^{\ell}\left(A_{i}\right)>1$ (meaning that $\left.\left|A_{i}\right| \geq 3^{\ell+1}\right)$ for each $i \leq k$. We now inductively construct $(k+1)$-tuples $\left(A_{0}^{j}, \ldots, A_{k}^{j}\right)$ $\left(j \leq n:=k(k+1) / 2\right.$ where $A_{i}^{0}=A_{i}$ for each $i \leq k$, and at stage $j<n$ we handle a distinct pair $\left(i^{0}, i^{1}\right)$ with $i^{0}<i^{1} \leq k$ so that

- $A_{i^{0}}^{j+1} \subseteq A_{i^{0}}^{j},\left|A_{i^{0}}^{j+1}\right| \geq\left|A_{i^{0}}^{j}\right| / 3$;

- $A_{i^{0}}^{j+1} \subseteq A_{i^{1}}^{j},\left|A_{i^{1}}^{j+1}\right| \geq \mid A_{i 1}^{j} 1 / 3$; and

- $A_{i^{0}}^{j+1} \cap A_{i^{1}}^{j+1}=\emptyset$.

(and $A_{i}^{j+1}=A_{i}^{j}$ for all other $i \leq k$ ). As $\left|A_{i^{0}}\right| \geq 3^{\ell+1}$ it follows by the induction that $\left|A_{i^{0}}^{j}\right| \geq 3$, and similarly $\left|A_{i^{1}}^{j}\right| \geq 3$, and so it is possible to partition the intersection $A_{i^{0}}^{j} \cap A_{i^{1}}^{j}$ into $Y \cup Z$ so that $\left|A_{i^{0}}^{j} \backslash Y\right| \geq\left|A_{i^{0}}^{j}\right| / 3$ and $\left|A_{i^{1}}^{j} \backslash Z\right| \geq \mid A_{i^{1}}^{j} 1 / 3$. We may then take $A_{i^{0}}^{j+1}:=A_{i^{0}}^{j} \backslash Y$ and $A_{i^{1}}^{j+1}:=A_{i^{1}}^{j} \backslash Z$.

After these steps, set $B_{i}:=A_{i}^{n}$ for each $i \leq k$. It is clear that the $B_{i}$ are pairwise disjoint (since if $i^{0}<i^{1} \leq k$ at some stage $j$ we would have handled this pair, meaning that $A_{i^{0}}^{j+1} \cap A_{i^{1}}^{j+1}=\emptyset$, but $B_{i^{0}} \subseteq A_{i^{0}}^{j+1}$ and $B_{i^{1}} \subseteq A_{i^{1}}^{j+1}$ ). As each $A_{i}$ was modified at most $k$ times in the inductive construction it follows that $\left|B_{i}\right| \geq\left|A_{i}\right| / 3^{k}$, and so $\mu^{\ell}\left(B_{i}\right)=\log _{3}\left(\left|B_{i}\right|\right) /(\ell+1) \geq \log _{3}\left(\left|A_{i}\right| / 3^{k}\right) /(\ell+1) \geq \log _{3}\left(\left|A_{i}\right|\right)-\ell /(\ell+1) \geq \mu^{\ell}\left(A_{i}\right)-1$.

Suppose now that for each $j \in J$ we have a subatomic family $\mathrm{K}_{j}$ living on a finite set $\operatorname{POSS}_{j}$. We can now define the atoms built from the subatoms.

Definition 2.2.3 - An atomic creature, or atom, a consists of a sequence $\left(x_{j}\right)_{j \in J}$ where $x_{j}$ is a $\mathrm{K}_{j}$-subatom for all $j \in J$.

- The norm of an atom $\mathfrak{a}=\left(x_{j}\right)_{j \in J}$, nor $(\mathfrak{a})$, is the maximal $r$ for which there is a set $A \subseteq J$ with $\mu^{\ell}(A) \geq r$ and nor $\left(x_{j}\right) \geq r$ for all $j \in A$. We say that such an $A$ "witnesses the norm" of $\mathfrak{a}$.

So the norm of an atom is large if there is a "large" subset $A$ of $J$ such that all subatoms in $A$ are "large".

The following easy fact will be useful later.

Fact 2.2.4 Suppose $A \subseteq J$ witnesses the norm of an atom $\mathfrak{a}=\left(x_{j}\right)_{j \in J}$, and let $\mathfrak{b}=$ $\left(y_{j}\right)_{j \in J}$ be any atom which agrees with $\mathfrak{a}$ on all indices in $A$. Then $\operatorname{nor}(\mathfrak{b}) \geq \operatorname{nor}(\mathfrak{a})$. In particular, if $\operatorname{nor}\left(y_{j}\right) \leq \operatorname{nor}\left(x_{j}\right)$ for all $j \notin A$, then $\operatorname{nor}(\mathfrak{b})=\operatorname{nor}(\mathfrak{a})$.

\subsection{Sacks columns}

Given a (finite) tree $T$, its splitting-size, $\operatorname{nor}_{\text {split }}(T)$, is defined as the maximal $\ell \in \omega$ such that there is a subset $S \subseteq T$ (with the induced order) which is order isomorphic to the complete binary tree $2^{\leq \ell}$ (of height $\ell$ with $2^{\ell}$ many leaves). Equivalently, $2^{\leq \ell}$ order-embeds into $T$.

Given a finite subset $I$ of $\omega$ and $F \subseteq 2^{I}$, we can identify $F$ with the tree of its restrictions $T_{F}=F \cup\{\eta\lceil n: \eta \in F, n \in I\}$ (a tree of partial functions from $I$ to 2, ordered by inclusion). We write $\operatorname{nor}_{\text {split }}(F)$ for nor split $\left(T_{F}\right)$.

The following establishes a basic combinatorial fact about this norm. 
Definition and Lemma 2.3.1 There exists a function $f$ with the following properties.

- For each $j, n, c$, whenever $\left(2^{f(j, n, c)}\right)^{j}$ is colored with $c$ colors, there are subsets $A_{1}, \ldots, A_{j}$ of $2^{f(j, n, c)}$ such that the set $A_{1} \times \cdots \times A_{j}$ is homogeneous, and $\operatorname{nor}_{\text {split }}\left(A_{i}\right) \geq n$ for all $i$. 5,6

- $f$ is monotone in each argument.

Proof We define $f(j, n, c)$ recursively on $j$ by $f(1, n, c)=n \cdot c$, and $f(j+1, n, c)=$ $f\left(1, n, c^{2^{j \cdot f(j, n, c)}}\right)=n \cdot c^{2^{j \cdot f(j, n, c)}}$. Note that $f(j, n, 1)=n$, and clearly any coloring $\pi:\left(2^{n}\right)^{j} \rightarrow 1$ is constant. We may then assume that $c>1$ for the remainder of the proof.

We first show by induction on $c$ that $f(1, n, c)$ is as required. Suppose that $f(1, n, c)$ works for some $c \geq 1$, and let $\pi: 2^{n \cdot(c+1)} \rightarrow c+1$ be a coloring. For $\eta \in 2^{n}$, let $[\eta]:=\left\{v \in 2^{n+c \cdot n}: \eta \subseteq v\right\}$. Note that $\operatorname{nor}_{\text {split }}([\eta])=2^{c \cdot n}$ for each $\eta \in 2^{n}$. If there is an $\eta \in 2^{n}$ such that $\pi \uparrow[\eta]$ omits one of $0, \ldots, c$, then $\pi \uparrow[\eta]$ is a coloring with at most $c$ colors, and so there must be an $A \subseteq[\eta] \subseteq 2^{n+c \cdot n}$ such that $\operatorname{nor}_{\text {split }}(A) \geq n$ and $\pi \uparrow A$ is constant.

Otherwise, for each $\eta \in 2^{n}$ there is an $v_{\eta} \in[\eta]$ such that $\pi\left(v_{\eta}\right)=0$. It follows that $A:=\left\{v_{\eta}: \eta \in 2^{n}\right\}$ has splitting size $n$, and $\pi \uparrow A$ is constantly 0 .

Assume that $f(j, n, c)$ satisfies the desired property for some $j \geq 1$. Set $p:=$ $f(j, n, c)$ and $q:=c^{2^{j \cdot p}}$, so that $f(j+1, n, c)=n \cdot q=f(1, n, q)$. Suppose $\pi:\left(2^{n \cdot q}\right)^{j+1} \rightarrow c$ is a coloring. Define $T:=\left\{\eta \in 2^{n \cdot q}: \eta \uparrow[p, n \cdot q)\right.$ is constantly 0$\}$. Since $c \geq 2$ it follows that $p<n \cdot q$, and so $\operatorname{nor}_{\text {split }}(T)=p$. For $\eta \in 2^{n \cdot q}$ define $\pi_{\eta}: T^{j} \rightarrow c$ by $\pi_{\eta}\left(\eta_{1}, \ldots, \eta_{j}\right)=\pi\left(\eta_{1}, \ldots, \eta_{j}, \eta\right)$. Note that the mapping $\eta \mapsto \pi_{\eta}$ is a coloring of $2^{n \cdot q}$ by at most $c^{\left(2^{p}\right)^{j}}=q$ many colors. By the above it follows that there is an $A_{j+1} \subseteq 2^{n \cdot q}$ and a $\pi^{*}: T^{j} \rightarrow c$ such that nor $_{\text {split }}\left(A_{j+1}\right) \geq n$ and $\pi_{\eta}=\pi^{*}$ for each $\eta \in A_{j+1}$.

Then as $\pi^{*}$ is a coloring of $T^{j}$ by at most $c$ colors, and as $\operatorname{nor}_{\text {split }}(T)=p=$ $f(j, n, c)$ by hypothesis for each $i \leq j$ there are $A_{i} \subseteq T \subseteq 2^{n \cdot q}$ with nor split $\left(A_{i}\right) \geq n$ (for $i \leq j$ ) such that $A_{1} \times \cdots \times A_{j}$ is homogeneous for $\pi^{*}$. It then follows that $A_{1} \times \cdots \times A_{j} \times A_{j+1}$ is homogeneous for $\pi$.

Definition 2.3.2 Suppose that $I$ is a nonempty (finite) interval in $\omega$. By a Sacks column on $I$ we mean a nonempty $\mathfrak{s} \subseteq 2^{I}$. We say that another Sacks column $\mathfrak{s}^{\prime}$ on $I$ is stronger than $\mathfrak{s}$, and write $\mathfrak{s}^{\prime} \leq \mathfrak{s}$, if $\mathfrak{s}^{\prime} \subseteq \mathfrak{s}$.

We can naturally take products of columns that are stacked above each other.

Definition 2.3.3 Let $\mathfrak{s}_{1}$ be a Sacks column on an interval $I_{1}$ and let $\mathfrak{s}_{2}$ be a Sacks column on an interval $I_{2}$. If $\min \left(I_{2}\right)=\max \left(I_{1}\right)+1$, then the product $\mathfrak{s}^{\prime}=\mathfrak{s}_{1} \otimes \mathfrak{s}_{2}$ is the Sacks column on $I_{1} \cup I_{2}$ defined by $f \in \mathfrak{s}^{\prime}$ iff $f \uparrow I_{1} \in \mathfrak{s}_{1}$ and $f \uparrow I_{2} \in \mathfrak{s}_{2}$.

\footnotetext{
5 As in the case of the bigness of subatoms, only the number of "colors" of our coloring functions is of importance. Moreover, by the definition of the splitting norm it follows that $T_{1}, \ldots, T_{j}$ are trees each of splitting size at least $f(j, n, c)$ and $\pi: T_{1} \times \cdots \times T_{j} \rightarrow c$ is a coloring, then there are $A_{i} \subseteq T_{i}(i \leq j)$ such that nor ${ }_{\text {split }}\left(A_{i}\right) \geq n$ for each $i$ and $\pi \uparrow A_{1} \times \cdots \times A_{j}$ is constant.

6 If $j=1$ this condition becomes whenever $2^{f(1, n, c)}$ is colored with $c$ colors there is a homogeneous subset $A$ of $2^{f(1, n, c)}$ such that $\operatorname{nor}_{\text {split }}(A) \geq n$.
} 
Iterating this, we can take products of finitely many properly stacked ${ }^{7}$ Sacks columns.

We now define the norm of a Sacks column $\mathfrak{s}$ on an interval $I$. Actually, we define a family of norms, using two parameters $B$ and $m$. Later, we will virtually always use values of $B$ and $m$ determined by $\min (I)$; more details will come in Sects. 2.5 and 4 .

Definition 2.3.4 nor $\operatorname{Sacks}_{\text {Sack }}^{B, m}(\mathfrak{s}) \geq n$ iff $n=0$ or nor $_{\text {split }}(\mathfrak{s}) \geq F_{m}^{B}(n)$ where $F_{m}^{B}: \omega \rightarrow \omega$ is defined as follows: $F_{m}^{B}(0)=1$ and $F_{m}^{B}(n+1)=f\left(m, F_{m}^{B}(n), B\right)$, where we use the function $f$ of Definition 2.3.1.

In other words,

$$
\operatorname{nor}_{\text {Sacks }}^{B, m}(\mathfrak{s})=\max \left(\left\{n \in \omega: F_{m}^{B}(n) \leq \operatorname{nor}_{\text {split }}(\mathfrak{s})\right\} \cup\{0\}\right) .
$$

The exact definition of this norm will not be important in the rest of the paper; we will only require the following properties.

Lemma 2.3.6 (1) If $\mathfrak{s ,} \mathfrak{s}^{\prime}$ have the same splitting-size, then $\operatorname{nor}_{\text {Sacks }}^{B, m}\left(\mathfrak{s}^{\prime}\right)=\operatorname{nor}_{\text {Sacks }}^{B, m}(\mathfrak{s})$.

(2) If $\mathfrak{s}^{\prime} \leq \mathfrak{s}, B^{\prime} \geq B$ and $m^{\prime} \geq m$, then $\operatorname{nor}_{\text {Sacks }}^{B^{\prime}, m^{\prime}}\left(\mathfrak{s}^{\prime}\right) \leq \operatorname{nor}_{\text {Sacks }}^{B, m}(\mathfrak{s})$.

(3) $\operatorname{nor}_{\text {Sacks }}^{B, m}\left(\mathfrak{s}_{1} \otimes \cdots \otimes \mathfrak{s}_{n}\right) \geq \operatorname{nor}_{\text {Sacks }}^{B, m}\left(\mathfrak{s}_{i}\right)$ for all $1 \leq i \leq n$.

(4) If $I$ is large (with respect to $B$ and $m$ ), then $\operatorname{nor}_{\text {Sacks }}^{B, m}\left(2^{I}\right)$ will be large. More precisely, given $a \in \omega$, if $|I|>F_{m}^{B}(a)$, then $\operatorname{nor}_{\text {Sacks }}^{B, m}\left(2^{I}\right) \geq a$.

(5) We will later use the following simple (but awkward) consequence. Fix properly stacked intervals $I, I^{\prime}$ and a Sacks column $\mathfrak{s}$ on $I \cup I^{\prime}$. Then there is an $\tilde{\mathfrak{s}} \leq \mathfrak{s}$ such that

$$
\operatorname{nor}_{\text {Sacks }}^{B, m}(\tilde{\mathfrak{s}}) \geq \min \left(\operatorname{nor}_{\text {Sacks }}^{B, m}(\mathfrak{s}), \operatorname{nor}_{\text {Sacks }}^{B, m}\left(2^{I}\right)\right)
$$

and $|\tilde{\mathfrak{s}}| \leq\left|2^{I}\right|$.

(6) (Bigness) For $i<m$, fix Sacks columns $\mathfrak{s}_{i}$ such that nor $_{\text {Sacks }}^{B, m}\left(\mathfrak{s}_{i}\right) \geq n+1$.

Then for any "coloring" function $\pi: \prod_{i<m} \mathfrak{s}_{i} \rightarrow B$ there are Sacks columns $\mathfrak{s}_{i}^{\prime} \leq \mathfrak{s}_{i}$ with nor Sacks $_{i}^{B, m}\left(\mathfrak{s}_{i}^{\prime}\right) \geq n$ such that $\pi$ is constant on $\prod_{i<m} \mathfrak{s}_{i}^{\prime}$.

Proof For (5), just prune all unnecessary branches. In more detail, note that nor $_{\text {split }}\left(2^{I}\right)=|I|$, and that nor Sacks $^{B, m}$ is determined by the splitting-size nor split $_{\text {. So }}$ we have to find $\tilde{\mathfrak{s}} \subseteq \mathfrak{s}$ with splitting size $r:=\min \left(\operatorname{nor}_{\text {split }}(\mathfrak{s}),|I|\right)$. Obviously we can find the binary tree $2^{\leq r}$ inside $\mathfrak{s}$ (as a suborder). Extend each of its maximal elements (uniquely), and take the downwards closure. This gives $\tilde{\mathfrak{s}}$.

(6) follows immediately from Lemma 2.3.1. We have $\operatorname{nor}_{\text {split }}\left(\mathfrak{s}_{i}\right) \geq F_{m}^{B}(n+1)=$ $f\left(m, F_{m}^{B}(n), B\right)$; so by the characteristic property of the function $f$, for any coloring function $\pi: \prod_{i<m} \mathfrak{s}_{i} \rightarrow B$ there are Sacks columns $\mathfrak{s}_{i}^{\prime} \leq \mathfrak{s}_{i}$ with nor $_{\text {split }}\left(\mathfrak{s}_{i}^{\prime}\right) \geq F_{m}^{B}(n)$ such that $\pi$ is constant on $\prod_{i<m} \mathfrak{s}_{i}^{\prime}$. So nor $\operatorname{Sacks}^{B, m}\left(\mathfrak{s}_{i}^{\prime}\right) \geq n$.

\footnotetext{
7 Sacks columns $\mathfrak{s}_{1}, \ldots, \mathfrak{s}_{n}$ on intervals $I_{1}, \ldots, I_{n}$, respectively, are called properly stacked if $\min \left(I_{i+1}\right)=$ $\max \left(I_{i}\right)+1$ for each $i<n$.
} 
Fig. 2 Diagram of the sublevels at level $\ell$, with the Sacks sublevel $(\ell,-1)$ occurring "before" the subatomic sublevels $(\ell, 0),(\ell, 1), \ldots,\left(\ell, J_{\ell}-1\right)$

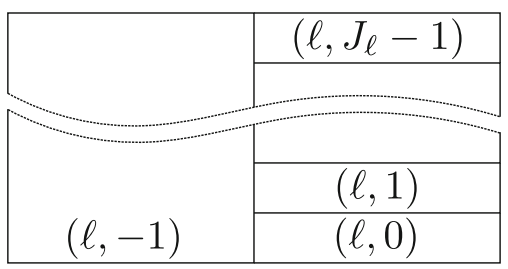

\subsection{Setting the stage}

We fix for the rest of this paper a nonempty (index) set $\Xi$. We furthermore assume that $\Xi$ is partitioned into subsets $\Xi_{1 \mathrm{~s}}, \Xi_{1 i}, \Xi_{\mathrm{sk}}\left(\Xi_{1 \mathrm{i}}\right.$ is nonempty, but $\Xi_{l_{\mathrm{s}}}$ and $\Xi_{\mathrm{sk}}$ could be empty). For each $\xi \in \Xi$, we say that $\xi$ is of type lim-sup, lim-inf or Sacks if $\xi$ is an element of $\Xi_{l_{\mathrm{s}}}, \Xi_{l_{\mathrm{i}}}$, or $\Xi_{\mathrm{sk}}$, respectively. We set $\Xi_{\text {non-sk }}:=\Xi_{l_{\mathrm{s}}} \cup \Xi_{l_{\mathrm{i}}}=\Xi \backslash \Xi_{\mathrm{sk}}$. level.

Our forcing will "live" on $\Xi \times \omega$. For $(\xi, \ell) \in \Xi \times \omega$ we call $\xi$ the index and $\ell$ the

The "frame" of the forcing will be as follows.

Definition 2.4.1 (1) (For the "Sacks part".) We fix a sequence $\left(I_{\mathrm{sk}, \ell}\right)_{\ell \in \omega}$ of properly stacked intervals in $\omega .{ }^{8}$ For simplicity we further assume that $\min \left(I_{\mathrm{sk}, 0}\right)=$ 0 . Given natural numbers $\ell<m$ we set $I_{\mathrm{sk},[\ell, m)}:=\bigcup_{\ell \leq h<m} I_{\mathrm{sk}, h}=$ $\left[\min \left(I_{\mathrm{Sk}, \ell}\right), \min \left(I_{\mathrm{Sk}, m}\right)\right)$. A Sacks column on $I_{\mathrm{Sk},[\ell, m)}$ is also called a "Sacks column between $\ell$ and $m$ ".

(2) We fix for each level $\ell \in \omega$ some $J_{\ell} \in \omega \backslash\{0\}$. A sublevel is a pair $(\ell, j)$ for $\ell \in \omega$ and $j \in J_{\ell} \cup\{-1\}$. (The sublevel $(\ell,-1)$ will be associated with the Sacks part at level $\ell$.) We will usually denote sublevels by $\mathbf{u}$ or $\mathbf{v}$.

(3) We say $\mathbf{v}$ is below $\mathbf{u}$, or $\mathbf{v}<\mathbf{u}$, if $\mathbf{v}$ lexicographically precedes $\mathbf{u}$. Note that this order has order type $\omega$.

(4) A sublevel $(\ell,-1)$ is called a Sacks sublevel; all other sublevels are called subatomic. Instead of $(\ell,-1)$ we will sometimes just write "the sublevel $\ell$ ", and we sometimes just write " $\mathbf{v}$ is below $\ell$ " instead of $\mathbf{v}<(\ell,-1)$.

(5) (For the "non-Sacks part".) For each subatomic sublevel $\mathbf{u}$ and index $\xi \in \Xi_{\text {non-sk }}$ we fix a subatomic family $\mathrm{K}_{\xi, \mathbf{u}}$ living on a finite set $\operatorname{POSS}_{\xi, \mathbf{u}}$.

(6) For each level $\ell \in \omega$ and index $\xi \in \Xi_{\text {non-sk }}$, each sequence $\left(x_{j}\right)_{j \in J_{\ell}}$ with $x_{j} \in$ $\mathrm{K}_{\xi, \mathbf{u}}$ constitutes (as in 2.2.3) an atom $\mathfrak{a}$, where we use $\ell$ as the parameter in $\mu^{\ell}$ for the definition of the norm of the atom (Fig. 2).

To be able to use this frame to construct a reasonable (in particular, proper) forcing, we will have to add several additional requirements of the following form. The Sacks intervals $I_{\mathrm{sk}, \ell}$ (that "appear" at sublevel $\ell$ ) are "large" with respect to everything that was constructed in sublevels $\mathbf{v}$ below $\ell$; and the subatoms at a subatomic sublevel u have "large" bigness with respect to everything that was constructed at sublevels $\mathbf{v}<\mathbf{u}$. The complete construction with all requirements will be given in Sect. 4 .

${ }^{8}$ I.e., $I_{\mathrm{sk}, \ell}=\left[\min \left(I_{\mathrm{sk}, \ell}\right), \min \left(I_{\mathrm{sk}, \ell+1}\right)\right)$ for all $\ell \in \omega$. 


\subsection{Compound creatures}

We can now define compound creatures, which are made up from subatomic creatures and Sacks columns.

Definition 2.5.1 A compound creature $\mathfrak{c}$ consists of

(1) natural numbers $m^{\text {dn }}<m^{\text {up; }}$

(2) a nonempty, finite ${ }^{9}$ subset supp of $\Xi$

(3) for each $\xi \in \operatorname{supp} \cap \Xi_{\text {sk }}$ a Sacks column $\mathfrak{c}(\xi)$ between $m^{\text {dn }}$ and $m^{\text {up; }}$;

(4) for each $\xi \in \operatorname{supp} \cap \Xi_{\text {non-sk }}$ and each subatomic sublevel $\mathbf{u}=(\ell, j)$ with $m^{\mathrm{dn}} \leq \ell<m^{\text {up }}$ a subatom $\mathfrak{c}(\xi, \mathbf{u}) \in \mathrm{K}_{\xi, \mathbf{u}}$; and

(5) for each $m^{\mathrm{dn}} \leq \ell<m^{\text {up }}$ a real number $d(\ell) \geq 0$, called the "halving parameter" of $\mathfrak{c}$ at level $\ell) .{ }^{10}$

We additionally require "modesty": 11

(6) for each subatomic sublevel $\mathbf{u}$ with $m^{\mathrm{dn}}<\mathbf{u}<m^{\text {up }}$ there is at most one $\xi \in \operatorname{supp} \cap \Xi_{\text {non-sk }}$ such that the subatom $\mathfrak{c}(\xi, \mathbf{u})$ is not a singleton.

Note that by (4) for each level $\ell$ with $m^{\text {dn }} \leq \ell<m^{\text {up }}$ and each $\xi \in \operatorname{supp} \cap \Xi_{\text {non-sk }}$ there is a naturally defined atom $\mathfrak{c}(\xi, \ell):=(\mathfrak{c}(\xi,(\ell, j)))_{j \in J_{\ell}}$.

We also write $m^{\mathrm{dn}}(\mathfrak{c}), m^{\text {up }}(\mathfrak{c}), \operatorname{supp}(\mathfrak{c}), d(\mathfrak{c}, h)$.

We will use the following assumptions (later there will be more; a complete list will be given in Sect. 4).

\section{Assumption 2.5.2 Let $\ell \in \omega$.}

- We fix natural numbers $B(\ell)$ and maxposs $(<\ell)$, such that for each $k \leq \ell$ we have $B(k) \leq B(\ell)$ and $\operatorname{maxposs}(<k) \leq \operatorname{maxposs}(<\ell)$. (These parameters will be defined in Sect. 4.)

- We assume that $I_{\mathrm{sk}, \ell}$ is large enough so that there are Sacks trees of large norm. (More concretely, $\operatorname{nor}_{\mathrm{Sacks}}^{B(\ell), \ell}\left(2^{I_{\mathrm{sk}, \ell}}\right) \geq \ell$.)

- We assume that $J_{\ell}$ is large enough such that $\mu^{\ell}\left(J_{\ell}\right)$ is big. (More concretely, $\left.\mu^{\ell}\left(J_{\ell}\right) \geq 2^{\ell \cdot \operatorname{maxposs}(<\ell)}\right)$.

- We assume that for every $\xi \in \Xi_{\text {non-sk }}$ and $j \in J_{\ell}$ there is (at least) one subatom $x \in \mathrm{K}_{\xi,(\ell, j)}$ with $\operatorname{nor}(x) \geq 2^{\ell \cdot \operatorname{maxposs}(<\ell)}$.

Using these assumptions, we can now define the norm of a compound creature.

Definition 2.5.3 The norm of a compound creature $\mathfrak{c}$, nor $(\mathfrak{c})$, is defined to be the minimum of the following values.

\footnotetext{
9 We could assume without loss of generality that the size of supp is at most $m^{\mathrm{dn}}$. This will be shown in Lemma 3.4.3.

10 One could (without loss of generality, in some sense) restrict the halving parameter to a finite subset of the reals; then for fixed supp, $m^{\mathrm{dn}}, m^{\text {up }}$ there are only finitely many compound creatures.

11 Again, without this requirement, the resulting forcing poset would be equivalent.
} 
(1) The "width norm":

$$
\operatorname{nor}_{\text {width }}(\operatorname{supp}(\mathfrak{c})):=\frac{m^{\mathrm{dn}}(\mathfrak{c})}{|\operatorname{supp}(\mathfrak{c})|} \text {. }
$$

(2) For each $\xi \in \operatorname{supp}(\mathfrak{c}) \cap \Xi_{\mathrm{sk}}$ the "Sacks norm" at index $\xi$ :

$$
\operatorname{nor}_{\text {Sacks }}(\mathfrak{c}(\xi)):=\operatorname{nor}_{\text {Sacks }}^{B\left(m^{\mathrm{dn}}\right), m^{\mathrm{dn}}}(\mathfrak{c}(\xi))
$$

(with $m^{\mathrm{dn}}:=m^{\mathrm{dn}}(\mathfrak{c})$ ) as defined in 2.3.5.

(3) For each $\xi \in \operatorname{supp}(\mathfrak{c}) \cap \Xi_{\text {non-sk }}$ the "lim-sup norm" at index $\xi$ :

$$
\operatorname{nor}_{\text {limsup }}(\mathfrak{c}, \xi):=\max \left(\operatorname{nor}(\mathfrak{c}(\xi, h)): m^{\mathrm{dn}} \leq h<m^{\text {up }}\right) \text {. }
$$

(4) For each $m^{\text {dn }}(\mathfrak{c}) \leq h<m^{\text {up }}(\mathfrak{c})$ the "lim-inf norm" at level $h$ :

$$
\operatorname{nor}_{\liminf }^{\operatorname{maxposs}\left(<m^{\mathrm{dn}}\right)}(\mathfrak{c}, h):=\frac{\log _{2}(N-d(\mathfrak{c}, h))}{\operatorname{maxposs}\left(<m^{\mathrm{dn}}\right)},
$$

where $N:=\min \left\{\operatorname{nor}(\mathfrak{c}(\xi, h)): \xi \in \operatorname{supp}(\mathfrak{c}) \cap \Xi_{1 i}\right\} .{ }^{12,13}$

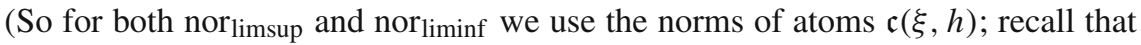
the level $h$ of this atom is used in Definition 2.2.3 of $\operatorname{nor}(\mathfrak{c}(\xi, h))$, more specifically, $\mu^{h}$ is used to measure the size of subsets of $J_{h}$.)

Remark 2.5.4 As supp(c) is nonempty, the width norm (and thus nor(c) as well) is at most $m^{\mathrm{dn}}(\mathfrak{c})$.

The assumptions imply the following.

Lemma 2.5.5 Fix $2<m^{\mathrm{dn}}<m^{\text {up }}$ and supp $\subseteq \Xi$ with $|\operatorname{supp}|<m^{\mathrm{dn}}$ and $\operatorname{supp} \cap \Xi_{\mathrm{Sk}}$, supp $\cap \Xi_{l_{i}}$, supp $\cap \Xi_{l_{\mathrm{s}}}$ all nonempty. Then there is a compound creature $\mathfrak{c}$ with $m^{\mathrm{dn}}(\mathfrak{c})=m^{\mathrm{dn}}, m^{\text {up }}(\mathfrak{c})=m^{\text {up }}, \operatorname{supp}(\mathfrak{c})=\operatorname{supp}$ such that $\operatorname{nor}(\mathfrak{c})=$ nor $_{\text {width }}(\operatorname{supp})$.

Proof We can first use for all subatoms and Sacks columns the "large" ones guaranteed by the assumptions. However, this will in general not satisfy modesty. So we just apply Lemma 2.2.2 at each $m^{\text {dn }} \leq \ell<m^{\text {up }}$, resulting (for each $\ell$ ) in disjoint sets $A_{\xi}^{\ell} \subseteq J_{\ell}$ for $\xi \in \operatorname{supp} \cap \Xi_{\text {non-sk }}$. We keep the large subatoms at the sublevels in $A_{\xi}^{\ell}$, and choose arbitrary singleton subatoms at other sublevels. Now we have a compound creature, whose norm is the minimum of the following:

- the width norm;

\footnotetext{
12 As usual, if the logarithm results in a negative number, or if we apply the logarithm to a negative number, we instead define the resulting norm to be 0 . So really we mean $\operatorname{nor}_{\liminf }^{\operatorname{maxposs}\left(<m^{\mathrm{dn}}\right)}(\mathfrak{c}, h):=$ $\frac{\log _{2}(\max (1, N-d(\mathfrak{c}, h))}{\operatorname{maxposs}\left(<m^{\mathrm{dn}}\right)}$.

13 The reason for the logarithm, and the use of the halving parameters, will become clear only in Sect. 5.2.
} 
- the (unchanged) Sacks norms, which are $\geq m^{\mathrm{dn}}>$ nor $_{\text {width }}$ (supp);

- the lim-sup norms, noting that all atoms at level $\ell$ have norm $\geq 2^{\ell \cdot \operatorname{maxposs}(<\ell)}-1 \geq$ $2^{m^{\mathrm{dn}} \cdot \operatorname{maxposs}\left(<m^{\mathrm{dn}}\right)}-1>$ nor $_{\text {width }}$ (supp), so all lim-sup norms drop by at most 1 ; and

- the lim-inf norms, which drop by an even smaller amount, due to the logarithm.

Fact 2.5.6 Let $\mathfrak{c}$ be a compound creature and $u \subseteq \operatorname{supp}(\mathfrak{c})$ such that $u \cap \Xi_{\mathrm{sk}}, u \cap \Xi_{1 i}$, $u \cap \Xi_{1 \mathrm{~s}}$ are all nonempty. Then the naturally defined $\mathfrak{c} \uparrow u$ is again a compound creature with norm at least nor(c).

Definition 2.5.7 A compound creature $\mathfrak{d}$ is "purely stronger" than $\mathfrak{c}$, if $\mathfrak{c}$ and $\mathfrak{d}$ have the same $m^{\mathrm{dn}}, m^{\text {up }}$, the same halving parameters, the same supp; and if for each $\xi \in \operatorname{supp} \cap \Xi_{\mathrm{sk}}$ the Sacks column $\mathfrak{d}(\xi)$ is stronger than $\mathfrak{c}(\xi)$ and for each subatomic sublevel $\mathbf{u}$ that appears in $\mathfrak{c}$ and $\xi \in \operatorname{supp} \cap \Xi_{\text {non-sk }}$ the subatom $\mathfrak{d}(\xi, \mathbf{u})$ is stronger than $\mathfrak{c}(\xi, \mathbf{u})$. (In other words, the only difference between $\mathfrak{c}$ and $\mathfrak{d}$ occurs at the Sacks columns and the subatoms, where they become stronger.)

For $r \geq 0$ we say that $\mathfrak{d}$ is " $r$-purely stronger" than $\mathfrak{c}$, if additionally nor $(\mathfrak{d}) \geq$ $\operatorname{nor}(\mathfrak{c})-r$.

To show that our forcing has the $\aleph_{2}-\mathrm{cc}$, we will use the following property.

Lemma 2.5.8 Fix two compound creatures $\mathfrak{c}_{1}$ and $\mathfrak{c}_{2}$ with same $m^{\mathrm{dn}}$ and $m^{\text {up }}$ and the same halving parameters, with disjoint supports, and such that $\operatorname{nor}\left(\mathfrak{c}_{1}\right), \operatorname{nor}\left(\mathfrak{c}_{2}\right)>$ $x$. Then there exists a compound creature $\mathfrak{d}$ with same $m^{\mathrm{dn}}$ and $m^{\mathrm{up}}$ and support $\operatorname{supp}\left(\mathfrak{c}_{1}\right) \cup \operatorname{supp}\left(\mathfrak{c}_{2}\right)$ such that $\operatorname{nor}(\mathfrak{d}) \geq \frac{x}{2}-1$ and $\mathfrak{d} \operatorname{supp}\left(\mathfrak{c}_{i}\right)$ is purely stronger than $\mathfrak{c}_{i}$ for $i=1,2$.

More generally, the same is true if $\mathfrak{c}_{1}$ and $\mathfrak{c}_{2}$ are not necessarily disjoint, but identical on the intersection $u:=\operatorname{supp}\left(\mathfrak{c}_{1}\right) \cap \operatorname{supp}\left(\mathfrak{c}_{2}\right)$, i.e., $\mathfrak{c}_{1}\left\lceil u=\mathfrak{c}_{2}\lceil u\right.$.

Proof Let $\mathfrak{d}^{\prime}$ be the "union" of $\mathfrak{c}_{1}$ and $\mathfrak{c}_{2}$, which is defined in the obvious way. ${ }^{14}$

As $\mathfrak{d}^{\prime}$ may not satisfy the modesty requirement (6) of Definition 2.5.1, we apply the procedure from the first part of the proof of Lemma 2.5.5 to ensure that the resulting object $\mathfrak{d}$ does. Then $\mathfrak{d}$ is a compound creature with norm $\geq \frac{x}{2}-1$. (The factor $\frac{1}{2}$ comes from doubling the size of the support, which decreases the width norm.)

\subsection{The elements (conditions) of the forcing poset $\mathbb{Q}$}

Definition 2.6.1 $\emptyset$ is the weakest condition. Any other condition $p$ consists of $\mathrm{w}^{p}$, $(p(h))_{h \in \mathrm{W}^{p}}$ and $t^{p}$ such that the following are satisfied.

- $\mathrm{w}^{p} \subseteq \omega$ is infinite.

- For each $h \in \mathrm{w}^{p}, p(h)$ is a compound creature whose $m^{\mathrm{dn}}$ is $h$, and whose $m^{\text {up }}$ is the $\mathrm{w}^{P}$-successor of $h$.

\footnotetext{
14 In particular $\operatorname{supp}\left(\mathfrak{d}^{\prime}\right)=\operatorname{supp}\left(\mathfrak{c}_{1}\right) \cup \operatorname{supp}\left(\mathfrak{c}_{2}\right)$, and the Sacks columns and subatoms of $\mathfrak{d}^{\prime}$ at index $\xi \in \operatorname{supp}\left(\mathfrak{d}^{\prime}\right)$ are exactly those from either $\mathfrak{c}_{1}$ or $\mathfrak{c}_{2}$, depending on whether $\xi \in \operatorname{supp}\left(\mathfrak{c}_{1}\right)$ or $\xi \in \operatorname{supp}\left(\mathfrak{c}_{2}\right)$.
} 
- For $h<h^{\prime}$ in $\mathrm{w}^{p}, \operatorname{supp}(p(h)) \subseteq \operatorname{supp}\left(p\left(h^{\prime}\right)\right)$,

- $\lim _{h \in \mathrm{W}} p(\operatorname{nor}(p(h)))=\infty$.

- We set $\operatorname{supp}(p):=\bigcup_{h \in \mathrm{w}^{p}} \operatorname{supp}(p(h))$ (a nonempty countable subset of $\Xi$ ).

- For $\xi \in \operatorname{supp}(p)$, we define $\operatorname{trklg}^{p}(\xi)$ (the "trunk length" at $\xi$ ) to be the minimal $h$ such that $\xi \in \operatorname{supp}(p(h))$.

- The "trunk" $t p$ is a function which assigns

- to each $\xi \in \operatorname{supp}(p) \cap \Xi_{\mathrm{sk}}$ and $\ell<\operatorname{trklg}^{p}(\xi)$ an element of $2^{I_{\mathrm{sk}, \ell}}$;

- to each $\xi \in \operatorname{supp}(p) \cap \Xi_{\text {non-sk }}$ and subatomic sublevel $\mathbf{u}$ below $\operatorname{trklg} p(\xi)$ an element of $\operatorname{POSS}_{\xi, \mathbf{u}}$.

Note that Assumption 2.5.2 guarantees that $\mathbb{Q}$ is nonempty (cf. Lemma 2.5.5).

Notation 2.6.2 Given $p \in \mathbb{Q}, h \in \mathrm{w}^{p}$ and $\ell$ which is $\geq h$ and less than the ${ }^{p}$ successor of $h$, and a sublevel $\mathbf{u}=(\ell, j)$ we use the following notations.

- $\operatorname{supp}(p, \mathbf{u})=\operatorname{supp}(p, \ell):=\operatorname{supp}(p(h))$.

- $d(p, \ell):=d(p(h), \ell)$ (the halving parameter of $p$ at level $\ell$ ).

- For $\xi \in \Xi_{\text {non-sk }} \cap \operatorname{supp}(p, \mathbf{u})$ and $j \neq-1$ we set $p(\xi, \mathbf{u}):=p(h)(\xi, \mathbf{u})$, the subatom located at index $\xi$ and sublevel $\mathbf{u}$.

- For $\xi \in \Xi_{\mathrm{sk}} \cap \operatorname{supp}(p(h))$ we set $p(\xi, h):=p(h)(\xi)$, the Sacks column at index $\xi$ starting at level $h$ (note that we require $h \in \mathrm{w}^{p}$ ).

\subsection{The set of possibilities}

We will now define the "possibilities" of a condition $p$, which give information about the possible value of the generic objects $y_{\xi}$ and which we will use to define the order of the forcing. The possibilities of a condition $p$ come from three sources, informally described below.

- The trunk $t^{p}$, where there is a unique possibility.

- The subatoms $p(\xi, \mathbf{u})$ (each with a set of possibilities, $\operatorname{poss}(p(\xi, \mathbf{u})))$.

- The Sacks columns $p(\xi, h)$ (which we interpret as a set of possible branches) which "live" between $h \in \mathrm{w}^{p}$ and the $\mathrm{w}^{p}$-successor $h^{+}$of $h$. The possibilities of the whole Sacks column have to be counted as belonging to the sublevel $(h,-1)$, i.e., we have to list them before the subatomic sublevel $(h, 0)$, even though their domain reaches up to just below $h^{+}$.

This property of the Sacks columns will make our notation quite awkward. As a consequence, the following section has the worst ratio of mathematical contents to notational awkwardness. Things will improve later on. We promise.

We first (in 2.7.1) describe a way to define the set of possibilities separately for each $\xi \in \operatorname{supp}(p)$; all possibilities then are the product over the $\xi$-possibilities.

Then (in 2.7.2) we will describe a variant in which possibilities at a sublevel $\mathbf{u}$ are defined, and all possibilities are a product over the u-possibilities.

Both versions result in the same set of possibilities (up to an awkward but canonical bijection; see Fact 2.7.3). The first version is more useful in formulating things such as "a stronger condition has as smaller set of possibilities", whereas the second is the notion that will actually be used later in proofs. 
Definition 2.7.1 Fix a condition $p$ and an index $\xi \in \operatorname{supp}(p)$.

- If $\xi \in \Xi_{\text {non-sk }}$, then for each subatomic sublevel $\mathbf{u}=(\ell, j)$ we define the set $\operatorname{poss}(p, \xi,=\mathbf{u})$ to be either the singleton $\left\{t^{p}(\xi, \mathbf{u})\right\}$ (if $\left.\ell<\operatorname{trklg}^{p}(\xi)\right)$, or $\operatorname{poss}(x)$ for the subatom $x:=p\left(\xi\right.$, u) (if $\left.\ell \geq \operatorname{trklg}^{p}(\xi)\right)$. (In either case $\operatorname{poss}(p, \xi,=\mathbf{u}) \subseteq$ $\operatorname{POSS}_{\xi, \mathbf{u}}$.)

We set $\operatorname{poss}(p, \xi,<\mathbf{u}):=\prod\{\operatorname{poss}(p, \xi,=\mathbf{v}): \mathbf{v}<\mathbf{u}$ is a subatomic sublevel $\}$.

- If $\xi \in \Xi_{\mathrm{Sk}}$ and $\mathbf{u}=(m, j)$ is a sublevel, we set $\ell$ to be either $m$ (if $j=-1$ and $m \in \mathrm{w}^{p}$ ), or the least number $>m$ in $\left\{0, \ldots, \operatorname{trklg}^{p}(\xi)-1\right\} \cup \mathrm{w}^{p}$, (otherwise). We then define $\operatorname{poss}(p, \xi,<\mathbf{u})$ to be the set of all functions $\eta \in 2^{\left[0, \min \left(I_{\mathrm{sk}, \ell}\right)\right)}$ compatible $^{15}$ with the trunk and the Sacks columns of $p$ at $\xi$.

- We set $\operatorname{poss}(p,<\mathbf{u})$ to be $\prod_{\xi \in \operatorname{supp}(p)} \operatorname{poss}(p, \xi,<\mathbf{u})$.

- Recall that we identify $\ell$ with the sublevel $(\ell,-1)$, so we can write $\operatorname{poss}(p,<\ell)$ instead of $\operatorname{poss}(p,<(\ell,-1))$.

Note that each possibility below $\mathbf{u}$ restricted to the non-Sacks part can be seen as a "rectangle" with width $\operatorname{supp}(p) \cap \Xi_{\text {non-sk }}$ and height $\mathbf{u}$; whereas the restriction to the Sacks part is a rectangle with height in $\mathrm{w}^{p}$ (which is generally above $\mathbf{u}$ ). So together this gives an "L-shaped" domain. Only in case $\mathbf{u}=(\ell,-1)$ for $\ell \in \mathrm{w}^{p}$ do we get a more pleasant overall rectangular shape.

In the following alternative definition we ignore a part of $p$ which is "trivial" because we have no freedom/choice left. More specifically, we ignore the trunk and singleton subatoms (but not singleton Sacks columns). Also, we do not first concentrate on some fixed index $\xi$, but directly define $\operatorname{poss}^{\prime}(p,=\mathbf{u})$ for certain sublevels $\mathbf{u}$.

Definition 2.7.2 We define the set $\operatorname{sblvls}(p)$ of "active" sublevels of $p$ by case distinction, and then for each $\mathbf{u} \in \operatorname{sblvls}(p)$ we define the object $\operatorname{poss}^{\prime}(p,=\mathbf{u})$.

- If $\mathbf{u}=(\ell,-1)$ is a Sacks sublevel, then $\mathbf{u} \in \operatorname{sblvls}(p)$ iff $\ell \in \mathrm{w}^{p}$. In this case we set $S:=\operatorname{supp}(p, \ell) \cap \Xi_{\mathrm{sk}} \neq \emptyset$, define $p(\mathbf{u})$ to be the sequence $(p(\xi, \ell))_{\xi \in S}$ of these Sacks columns, and set $\operatorname{poss}^{\prime}(p,=\mathbf{u})$ to be the product of this sequence.

- If $\mathbf{u}=(\ell, j)$ is a subatomic sublevel, then $\mathbf{u} \in \operatorname{sblvls}(p)$ iff $\ell \geq \min \left(\mathrm{w}^{p}\right)$ and if there is a non-singleton subatom at sublevel $\mathbf{u}$, say at index $\xi$. In this case according to the modesty condition (6) of Definition 2.5.1 this is the only nonsingleton subatom at $\mathbf{u}$. We call $\xi$ the "active index" at $\mathbf{u}$, set $p(\mathbf{u}):=p(\xi$, $\mathbf{u})$ (the "active subatom") and define $\operatorname{poss}^{\prime}(p,=\mathbf{u}):=\operatorname{poss}(p(\mathbf{u}))$.

So $\operatorname{sblvls}(p)$ is a subset (and thus suborder) of the set of all sublevels, also of order type $\omega$. We set $\operatorname{poss}^{\prime}(p,<\mathbf{u}):=\prod\left\{\operatorname{poss}^{\prime}(p,=\mathbf{v}): \mathbf{v}<\mathbf{u}, \mathbf{v} \in \operatorname{sblvls}(p)\right\}$.

The definition of the following bijection $\iota$ is easy to see/understand, but very awkward to formulate precisely, and hence left as an exercise.

Fact 2.7.3 There is a natural/canonical correspondence $\iota: \operatorname{poss}(p,<\mathbf{u}) \rightarrow$ $\operatorname{poss}^{\prime}(p,<\mathbf{u})$. Given an $\eta \in \operatorname{poss}(p,<\mathbf{u})$, we first omit from $\eta$ all the "trivial" information contained in the trunk and in the singleton subatoms; and then "relabel" the resulting sequence (instead of a sequence indexed by elements of $\xi$ we wish to have one indexed by elements of $\operatorname{sblvls}(p)$ ).

$\overline{15}$ In more detail, for each $h<\ell$ an element of $\{0, \ldots, \operatorname{trklg} p(\xi)-1\} \cup{ }_{\mathrm{w}}^{p}$ if $h<\operatorname{trklg} p(\xi)$, then $\eta\left\lceil I_{\mathrm{Sk}, h}=t^{p}(\xi, h)\right.$, and otherwise $\eta\left\lceil I_{\mathrm{Sk},\left[h, h^{\prime}\right)} \in p(\xi, h)\right.$, where $h^{\prime}$ is the ${ }^{p}$-successor of $h$. 
Later in this paper we will not distinguish between poss and poss'; actually, we will mostly use poss' ${ }^{\prime}$, and often use the following trivial observation.

Fact 2.7.4 For $\mathbf{v}<\mathbf{u}$ in $\operatorname{sblvls}(p)$,

$$
\operatorname{poss}^{\prime}(p,<\mathbf{u})=\operatorname{poss}^{\prime}(p,<\mathbf{v}) \times \operatorname{poss}^{\prime}(p,=\mathbf{v}) \times \operatorname{poss}^{\prime}(p,>\mathbf{v}),
$$

where we set $\operatorname{poss}^{\prime}(p,>\mathbf{v}):=\prod_{\mathbf{v}^{\prime} \in \operatorname{sblvls}(p), \mathbf{v}<\mathbf{v}^{\prime}<\mathbf{u}} \operatorname{poss}\left(p,=\mathbf{v}^{\prime}\right)$.

$\operatorname{poss}^{\prime}(p,=\mathbf{v})$ is a product of Sacks columns if $\mathbf{v}$ is Sacks, otherwise it is $\operatorname{poss}(x)$ for the active subatom at $\mathbf{v}$.

\subsection{The order of the forcing}

Definition 2.8.1 A condition $q$ is stronger than $p$, written $q \leq p$, iff the following conditions hold.

(1) $\mathrm{w}^{q} \subseteq \mathrm{w}^{p}$.

(2) $\operatorname{supp}(p) \cap \operatorname{supp}(q(h))=\operatorname{supp}(p(h))$ for each $h \in \mathrm{w}^{q} \cdot{ }^{16}$

(3) The trunk $t^{q}$ of $q$ extends the trunk $t^{p}$ of $p$ and is "compatible" with $p$ in the sense that for each $\xi \in \operatorname{supp}(p)$ the $\operatorname{singleton} \operatorname{poss}\left(q, \xi,<\operatorname{trklg}^{q}(\xi)\right)$ is a subset of $\operatorname{poss}\left(p, \xi,<\operatorname{trklg}^{q}(\xi)\right){ }^{17}$ (I.e., the subatoms and Sacks columns of $p$ that disappeared have become part of the trunk of $q$ which is compatible with the respective possibilities of $p$.)

(4) If $\xi \in \operatorname{supp}(p) \cap \Xi_{\text {non-sk }}$ and $\mathbf{u}$ is a subatomic sublevel above $\operatorname{trklg}^{q}(\xi)$, then the subatom $q(\xi, \mathbf{u})$ is stronger than $p(\xi, \mathbf{u})$.

(5) If $\xi \in \operatorname{supp}(p) \cap \Xi_{\mathrm{sk}}$ and $h \in \mathrm{w}^{q}$ such that $h \geq \operatorname{trklg}^{q}(\xi)$, then the Sacks column $q(\xi, h)$ is stronger than (i.e., a subset of) the product of the Sacks columns $p(\xi, \ell)$ for $\ell \in \mathrm{w}^{p}, h \leq \ell<h^{+}$, where $h^{+}$is the $\mathrm{w}^{q}$-successor of $h$.

(6) The halving parameters do not decrease; i.e., $d(q, \ell) \geq d(p, \ell)$ for all $\ell \in \omega$ with $\ell \geq \min \left(\mathrm{w}^{q}\right)$.

\section{Some simple properties of $\mathbb{Q}$}

\subsection{Increasing the trunk}

We now introduce an obvious way to strengthen a condition: increase the trunk.

Definition 3.1.1 Given $\ell \in \mathrm{w}^{p}$ and $\eta \in \operatorname{poss}(p,<\ell)$, we define $p \wedge \eta$ to be the condition $q$ resulting from replacing the compound creatures below $\ell$ with the trunk $\eta$. More formally, $\mathrm{w}^{q}:=\mathrm{w}^{p} \backslash \ell, q(k):=p(k)$ for all $k \in \mathrm{w}^{q}, t^{q}(\xi, \mathbf{u}):=\eta(\xi, \mathbf{u})$ for each $\xi \in \operatorname{supp}(p) \cap \Xi_{\text {non-sk }}$ and each subatomic $\mathbf{u}<\ell$, and $t^{q}(\xi, h)=\eta(\xi)\left\lceil I_{\mathrm{Sk}, h}\right.$ for each $\xi \in \operatorname{supp}(p) \cap \Xi_{\mathrm{sk}}$ and each $h<\ell$.

\footnotetext{
16 This condition in particular implies that $\operatorname{supp}(q(h)) \supseteq \operatorname{supp}(p(h))$ for each $h \in \mathrm{w}^{q}$, $\operatorname{that} \operatorname{trklg}^{q}(\xi)=$ $\min \left\{\ell \in \mathrm{w}^{q}: \ell \geq \operatorname{trklg}^{p}(\xi)\right\}$ for each $\xi \in \operatorname{supp}(p)$, and that $t^{q}$ is defined on a larger domain than $t^{p}$.

17 Equivalently, for any $\eta \in \operatorname{poss}\left(q,<\min \left(\mathrm{w}^{q}\right)\right)$, the restriction of $\eta$ to $\operatorname{supp}(p)$ is in $\operatorname{poss}\left(p,<\min \left(\mathrm{w}^{q}\right)\right)$.
} 
The definition of the order yields the following simple consequences.

Fact 3.1.2 Fix $p \in \mathbb{Q}$ and $\ell \in \mathrm{w}^{p}$.

- For $\eta \in \operatorname{poss}(p,<\ell), p \wedge \eta \leq p$.

- $\{p \wedge \eta: \eta \in \operatorname{poss}(p,<\ell)\}$ is predense below $p$.

- In particular, assuming that $p$ and $q$ are conditions that above some $\ell_{1}$ have the same $\mathrm{w}$ and the same compound creatures, ${ }^{18}$ and that $\operatorname{poss}\left(q,<\ell_{1}\right) \subseteq$ $\operatorname{poss}\left(p,<\ell_{1}\right)$, then $q \leq^{*}$ p. ${ }^{19}$

We can define a variant of $\wedge$, which works for any sublevel (not only those Sacks sublevels $\mathbf{u}=(\ell,-1)$ with $\left.\ell \in \mathrm{w}^{p}\right)$.

Definition 3.1.3 Given $\eta \in \operatorname{poss}(p,<\mathbf{u})$, we define $p \curlywedge \eta$ as the condition $q$ obtained by replacing the according parts of $p$ with the singleton subatoms (or singleton Sacks columns) given by $\eta$. More formally, the only possible differences between $p$ and $q$ are that for each subatomic sublevel $\mathbf{v}<\mathbf{u}$ and each $\xi \in \operatorname{supp}(p, \mathbf{v}) \cap \Xi_{\text {non-sk }}$ the subatomic creature $q(\xi, \mathbf{v})$ is the singleton subatom $\{\eta(\xi, \mathbf{v})\}$, and for each $\ell \in \mathrm{w}^{p}$ strictly below $\mathbf{u}$ and each $\xi \in \operatorname{supp}(p, \ell) \cap \Xi_{\mathrm{sk}}$ the Sacks column $p(\xi, \ell)$ is the singleton $\left\{\eta(\xi) \mid I_{\mathrm{sk},\left[\ell, \ell^{+}\right)}\right.$, where $\ell^{+}$is the $\mathrm{w}^{p}$-successor of $\ell$.

We can now define the generic sequence added by the forcing. (Note that the generic filter will generally not be determined by this sequence, due to additional information given by $\mathrm{w}$ and the halving parameters.)

Definition 3.1.4 For $\xi \in \Xi_{\text {non-sk }}$, let $\underset{\sim}{y_{\xi}}$ be (the name for)

$$
\left\{(\mathbf{u}, a): \mathbf{u} \text { a subatomic sublevel and }(\exists p \in G) t^{p}(\xi, \mathbf{u})=a\right\} .
$$

For $\xi \in \Xi_{\mathrm{Sk}}$, we set $y_{\xi}$ to be

$$
\bigcup\left\{t^{p}(\xi, \ell): p \in G, \ell<\operatorname{trklg}^{p}(\xi)\right\} .
$$

Fact 3.1.5 Let $\mathbf{u}$ be a sublevel.

- For $\eta \in \operatorname{poss}(p,<\mathbf{u}), p \curlywedge \eta \leq p$.

- If $\ell \in \mathrm{w}^{p}, \mathbf{u}=(\ell,-1)$ and $\eta \in \operatorname{poss}(p,<\ell)$, then $p \curlywedge \eta \leq{ }^{*} p \wedge \eta$ and $p \wedge \eta \leq p \curlywedge \eta$.

- $\{p \curlywedge \eta: \eta \in \operatorname{poss}(p,<\mathbf{u})\}$ is predense below $p$.

- $p \curlywedge \eta$ and $p \curlywedge \eta^{\prime}$ are incompatible if $\eta^{\prime}, \eta \in \operatorname{poss}(p,<\mathbf{u})$ are distinct.

- $p \curlywedge \eta$ forces that $\bar{y}$ extends $\eta$, i.e., that $y_{\xi}$ extends $\eta(\xi)$ for all $\xi \in \operatorname{supp}(p)$. In particular, $p$ forces that $\bar{y}$ extends $t^{p^{\tilde{y}}}$.

- $\eta \in \operatorname{poss}(p,<\mathbf{u})$ iff ${ }^{20} p$ does not force that $\eta$ is incompatible with the generic reals $\underset{\sim}{\bar{y}}$.

\footnotetext{
18 More formally, $\ell_{1} \in \mathrm{w}^{p}, \mathrm{w}^{p} \backslash \ell_{1}=\mathrm{w}^{q} \backslash \ell_{1}$, and $p(h)=q(h)$ for all $h \in \mathrm{w}^{p} \backslash \ell_{1}$. Note that this implies $\operatorname{supp}(p)=\operatorname{supp}(q)$.

${ }^{19}$ Here, $q \leq^{*} p$ means that $q$ forces that $p$ belongs to the generic filter. Equivalently, every $r \leq q$ is compatible with $p$.

${ }^{20}$ For the direction "right to left", which we will not need in this paper, we of course have to assume that $\eta$ has the right "format", i.e., $\eta=\prod_{\xi \in \operatorname{supp}(p)} \eta(\xi)$ and each $\eta(\xi)$ has the appropriate length/domain.
} 
- For $\eta \in \operatorname{poss}(p,<\mathbf{u}), p \Vdash$ “ $\bar{y}$ extends $\eta \Leftrightarrow p \curlywedge \eta \in G$.”

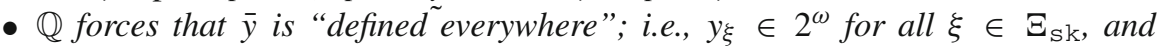
$y_{\eta}(\mathbf{u}) \in \operatorname{POSS}_{\xi, \mathbf{u}}$ is defined for all $\xi \in \Xi_{\text {non-sk }}$ and every subatomic sublevel $\mathbf{u}$.

Proof of the last item. Given a condition $p$ and $\xi \in \Xi$, we have to show that we can find a $q \leq p$ with $\xi \in \operatorname{supp}(q)$. This is shown just as Lemma 2.5.8, using at $\xi$ the large Sacks columns/subatoms guaranteed by 2.5.2. Then "increasing the trunk" shows that $y_{\xi}(n)$ is defined for all $n$.

Note that we can use the equivalent poss' (defined in 2.7.2) instead of poss. Formally, we could use the bijection $\iota$ of 2.7.3 and set $p \wedge \eta^{\prime}:=p \wedge \iota^{-1}\left(\eta^{\prime}\right)$ for $\eta^{\prime} \in \operatorname{poss}^{\prime}(p,<l)$ (and $p \curlywedge \eta^{\prime}:=p \curlywedge \iota^{-1}\left(\eta^{\prime}\right)$ for $\eta^{\prime} \in \operatorname{poss}^{\prime}(p,<\mathbf{u})$ ). But what we really mean is that for some $\eta^{\prime} \in$ poss' we can define $p \wedge \eta^{\prime}\left(p \curlywedge \eta^{\prime}\right)$ in the obvious and natural way; and this results in the same object as when using $p \wedge \eta(p \curlywedge \eta)$ for the $\eta \in$ poss that corresponds to $\eta^{\prime}$ (i.e., for $\eta=\iota^{-1}\left(\eta^{\prime}\right)$ ).

\subsection{The set of possibilities of stronger conditions}

If $q \leq p$, then $\operatorname{poss}(q,<\mathbf{u})$ is "morally" a subset of $\operatorname{poss}(p,<\mathbf{u})$ for any $\mathbf{u}$.

If we just consider a sublevel $(\ell,-1)$ for $\ell \in \mathrm{w}^{q}$ then this is literally true:

Assume that $q \leq p, \xi \in \operatorname{supp}(p)$ and $\ell \in \mathrm{w}^{q}$. Then $\operatorname{poss}(q, \xi,<\ell) \subseteq$ $\operatorname{poss}(p, \xi,<\ell)$.

In the general case it is more cumbersome to make this explicit for the Sacks part. However, we will only need the following.

Lemma 3.2.1 Given $q \leq p$ and $\eta \in \operatorname{poss}(q,<\mathbf{u})$ there is a unique $\eta^{\prime} \in \operatorname{poss}(p,<\mathbf{u})$ such that $q \curlywedge \eta \leq p \curlywedge \eta^{\prime}$.

Proof Uniqueness follows from the fact that $p \curlywedge \eta^{\prime}$ and $p \curlywedge \eta^{\prime \prime}$ are incompatible for distinct $\eta^{\prime}, \eta^{\prime \prime}$ in $\operatorname{poss}(p,<\mathbf{u})$.

We define $\eta^{\prime}(\xi)$ separately for each $\xi \in \operatorname{supp}(p)$. For $\xi \in \Xi_{\text {non-sk }}$ we just use $\eta^{\prime}(\xi):=\eta(\xi)$. So assume $\xi \in \Xi_{\mathrm{sk}}$. Let $k$ be the smallest element of $\mathrm{w}^{p}$ above $\mathbf{u}$.

- If $\mathbf{u}$ is below $\operatorname{trklg}^{p}(\xi)$ (and therefore also below $\operatorname{trklg}^{q}(\xi)$ ), then again we set $\eta^{\prime}(\xi):=\eta(\xi)$.

- If $\mathbf{u}$ is above $\operatorname{trklg}^{p}(\xi)$ but below $\operatorname{trklg}^{q}(\xi)$, then we extend $\eta(\xi)$ up to $k$ with the values given by the trunk $t^{q}$. This gives $\eta^{\prime}(\xi)$.

- If $\mathbf{u}$ is above $\operatorname{trklg}^{q}(\xi) \geq \operatorname{trklg}^{p}(\xi)$, then $\eta^{\prime}(\xi)$ is the restriction of $\eta(\xi)$ to $k$.

Remark 3.2.2 Note that $q \leq p$ does not imply $\operatorname{sblvls}(q) \subseteq \operatorname{sblvls}(p)$, as a previously "inactive" sublevel of $p$ can become active in $q$ (with active index outside of $\operatorname{supp}(p)$, of course). Also, $\mathbf{u}$ can be an active subatomic sublevel in both $p$ and $q$, but with different active indices. The "old" active subatom at $\xi$ can shrink to a singleton in $q$, while $q$ gains a new index with an active subatom (outside of $\operatorname{supp}(p)$ ). Because of this, it is even more cumbersome to formulate an exact version of "stronger conditions have fewer possibilities" for poss' than it is for poss. 


\section{$3.3 \$_{2}$ chain condition}

Lemma 3.3.1 Assuming $C H, \mathbb{Q}$ is $\aleph_{2}-c c$.

Proof Assume that $A=\left\{p_{i}: i \in \aleph_{2}\right\}$ is a set of conditions. By thinning out $A$ (only using $\mathrm{CH}$ and the $\Delta$-system lemma for families of countable sets), we may assume that there is a countable set $\Delta \subseteq \Xi$ such that for $p \neq q$ in $A$ the following hold:

- ${ }_{\mathrm{W}}^{p}=\mathrm{w}^{q}$;

- $d(p, \ell)=d(q, \ell)$ for all $\ell \geq \min \left(\mathrm{w}^{p}\right)$;

- $\Delta=\operatorname{supp}(p) \cap \operatorname{supp}(q)$, and, moreover, $\operatorname{supp}(p, \ell) \cap \Delta=\operatorname{supp}(q, \ell) \cap \Delta$ for all $\ell \in \mathrm{w}^{p}$; and

- $p$ and $q$ are identical on $\Delta$, i.e., for each $\ell \in \mathrm{w}^{p}$ the compound creatures $p(\ell)$ and $q(\ell)$ are identical on the intersection, as in Lemma 2.5.8; and the trunks agree on $\Delta$, i.e., $t^{p}(\xi, \ell)$ is the same as $t^{q}(\xi, \ell)$ for each $\xi \in \Delta \cap \Xi_{\mathrm{Sk}}$ and $\ell<h(\xi)$, and analogously for the subatomic sublevels.

As in Lemma 2.5.8 we can (for each $p, q \in A$ and $\ell \in \mathrm{w}^{p}$ ) find a compound creature $\mathfrak{d}(\ell)$ "stronger than" both $p(\ell)$ and $q(\ell)$. These creatures (together with the union of the trunks) form a condition stronger than both $p$ and $q$. Hence $A$ is not an antichain.

\subsection{Pruned conditions}

Let $p$ be a condition. All compound creatures $p(\ell)$ above some $\ell_{0}$ will have norm at least 1 . Note that by the definition of nor $_{\text {width }}$ this implies that $|\operatorname{supp}(p, \ell)| \leq \ell$.

The norm of a compound creature $\mathfrak{c}$ is at most $m^{\mathrm{dn}}$ (where we set $\left.m^{\mathrm{dn}}:=m^{\mathrm{dn}}(\mathfrak{c})\right)$. We assumed that nor ${ }_{\text {Sacks }}^{B\left(m^{\mathrm{dn}}\right), m^{\mathrm{dn}}}\left(2^{I_{\mathrm{sk}, m^{\mathrm{dn}}}}\right)$ is at least $m^{\mathrm{dn}}$. Let $\mathfrak{s}$ be any Sacks column in c. By Lemma 2.3.6(5) (using $I:=I_{\mathrm{sk}, m^{\mathrm{dn}}}$ and $I^{\prime}:=I_{\mathrm{sk},\left[m^{\mathrm{dn}}+1, m\right.} m^{\text {up })}$ ), there is an $\tilde{\mathfrak{s}} \subseteq \mathfrak{s}$ with $|\tilde{\mathfrak{s}}| \leq 2^{I_{\mathrm{sk},} m^{\mathrm{dn}}}$ and $\operatorname{nor}_{\text {Sacks }}^{B\left(m^{\mathrm{dn}}\right), m^{\mathrm{dn}}}(\tilde{\mathfrak{s}}) \geq \min \left(m^{\mathrm{dn}}, \operatorname{nor}_{\text {Sacks }}^{B\left(m^{\mathrm{dn}}\right), m^{\mathrm{dn}}}(\mathfrak{s})\right)$. So when we replace $\mathfrak{s}$ by $\tilde{\mathfrak{s}}$ in $\mathfrak{c}$, the norm of the compound creature does not change. Furthermore, if we replace all Sacks columns in $\mathfrak{c}$ with appropriate stengthenings, the resulting compound creature $\mathfrak{d}$ will be 0 -purely stronger than $\mathfrak{c} .^{21}$

This leads us to the following definitions.

Definition 3.4.1 We call a Sacks column $\mathfrak{s}$ between $\ell$ and $n$ Sacks-pruned if $|\mathfrak{s}| \leq$ $2^{\left|I_{\mathrm{sk}, \ell}\right|}$. A compound creature is Sacks-pruned if all its Sacks columns are. A condition $q$ is Sacks-pruned if $q(h)$ is Sacks-pruned for all $h \in \mathrm{w}^{q}$. A condition $p$ is pruned if it is Sacks-pruned and all compound creatures $p(h)$ have norm bigger than 1 .

Definition 3.4.2 A condition $q$ is purely stronger ( $r$-purely stronger) than $p$, if $\mathrm{w}^{q}=$ $\mathrm{w}^{p}, t^{q}=t^{p}$, and $q(\ell)$ is purely stronger ( $r$-purely stronger) than $p(\ell)$ for all $\ell \in \mathrm{w}^{q}$. (Note that this implies $q \leq p$.)

\footnotetext{
21 See Definition 2.5.7.
} 
For every condition $p$ there is a Sacks-pruned condition $q$ which is 0 -purely stronger than $p$. Given $p \in \mathbb{Q}$ Sacks-pruned, $\ell \in \mathrm{w}^{p}$ sufficiently large, and $\eta \in \operatorname{poss}(p,<\ell)$, the condition $q=p \wedge \eta<p$ is pruned.

In particular, we have the following.

Fact 3.4.3 - If $p$ is pruned, then $|\operatorname{supp}(p(h))|<h$ for all $h \in \mathrm{w}^{p}$.

- The set of pruned conditions in $\mathbb{Q}$ is dense.

\subsection{Gluing}

So far we have increased trunks to strengthen conditions, as well as taking disjoint unions and pure strengthenings. This subsection introduces two more methods of strengthening conditions.

Definition 3.5.1 A compound creature $\mathfrak{d}$ is the result of increasing the halving parameters in $\mathfrak{c}$, if $\mathfrak{d}$ and $\mathfrak{c}$ are identical except that for each $m^{\mathrm{dn}} \leq \ell<m^{\text {up }}$ we may have $d(\mathfrak{d}, \ell)>d(\mathfrak{c}, \ell)$.

Analogously, we define a condition $q$ to be the result of increasing the halving parameters in $p$. (Again, this implies $q \leq p$.)

Definition 3.5.2 We call a finite sequence of compound creatures $\mathfrak{c}_{1}, \ldots, \mathfrak{c}_{n}$ properly stacked if $m^{\text {up }}\left(\mathfrak{c}_{i}\right)=m^{\mathrm{dn}}\left(\mathfrak{c}_{i+1}\right)$ and $\operatorname{supp}\left(\mathfrak{c}_{i}\right) \subseteq \operatorname{supp}\left(\mathfrak{c}_{i+1}\right)$ for each $i<n$. Given such a sequence, we can glue it together to get the new creature $\mathfrak{d}=\operatorname{glue}\left(\mathfrak{c}_{1}, \ldots, \mathfrak{c}_{n}\right)$ with the following description.

- $m^{\mathrm{dn}}(\mathfrak{d})=m^{\mathrm{dn}}\left(\mathfrak{c}_{1}\right)$ and $m^{\text {up }}(\mathfrak{d})=m^{\text {up }}\left(\mathfrak{c}_{n}\right)$ (i.e., vertically the creature lives on the union of the levels of the old creatures).

- $\operatorname{supp}(\mathfrak{d})=\operatorname{supp}\left(\mathfrak{c}_{1}\right)$ (i.e., the rectangle-shape of the new creature is the result of taking the union of the old rectangles and cutting off the stuff that sticks out horizontally beyond the base).

- For $\xi \in \operatorname{supp}(\mathfrak{d}) \cap \Xi_{\text {non-sk }}$ and subatomic sublevels u between $m^{\mathrm{dn}}(\mathfrak{d})$ and $m^{\text {up }}(\mathfrak{d})$, the subatom $\mathfrak{d}(\xi, \mathbf{u})$ is $\mathfrak{c}_{i}(\xi, \mathbf{u})$ for the appropriate $i$.

- For $\xi \in \operatorname{supp}(\mathfrak{d}) \cap \Xi_{\mathrm{Sk}}$, the Sacks column $\mathfrak{d}(\xi)$ is defined as the product $\mathfrak{c}_{1}(\xi) \otimes$ $\cdots \otimes \mathfrak{c}_{n}(\xi)$.

By the definition of the norm (see 2.5.3), the monotonicity of $B$ and maxposs (Assumption 2.5.2) and Lemma 2.3.6(2),(3) we get

$$
\operatorname{nor}\left(\operatorname{glue}\left(\mathfrak{c}_{1}, \ldots, \mathfrak{c}_{n}\right)\right) \geq \min \left(\operatorname{nor}\left(\mathfrak{c}_{1}\right), \ldots, \operatorname{nor}\left(\mathfrak{c}_{n}\right)\right) .
$$

This gives another way to strengthen a condition $p$ : shrinking the set $\mathrm{w}$.

Definition 3.5.3 Given a condition $p$ and an infinite subset $U$ of ${ }_{\mathrm{w}}{ }^{p}$ such that $\min (U)=\min \left({ }^{p}{ }^{p}\right)$, we say that $q$ results from gluing $p$ along $U$ if

- $\mathrm{w}^{q}=U$;

- for $h \in \mathrm{w}^{q}$, if $h=h_{1}<h_{2}<\cdots<h_{n}$ enumerates the elements of $\mathrm{w}^{p}$ that are $\geq h$ and less than the $\mathrm{w}^{q}$-successor of $h$, then the compound creature $q(h)$ is glue $\left(p\left(h_{1}\right), \ldots, p\left(h_{n}\right)\right)$; and

- the new parts of the trunk are compatible with $p$. 
Note that $q$ is not uniquely determined by $p$ and $U$, as in general there are many choices to increase the trunk (in the last item). Of course, any such resulting $q$ is stronger than $p$.

We have now seen five specific ways to strengthen a condition. Actually, every $q \leq p$ can be obtained from $p$ by a combination of these methods. (We will not use the following fact, nor the subsequent remark, in the rest of the paper.)

Fact 3.5.4 For $p, q \in \mathbb{Q}, q \leq p$ iff there are $p_{1}, p_{2}, p_{3}$ and $p_{4}$ such that:

(1) $p_{1}$ results from increasing the trunk in $p$, i.e., $p_{1}=p \wedge \eta$ for some $\eta \in$ $\operatorname{poss}\left(p,<\min \left(\mathrm{w}^{q}\right)\right)$ (in fact, for the unique $\eta$ which is extended by $\left.t^{q}\right)$;

(2) $\quad p_{2} \leq p_{1}$ results from gluing $p_{1}$ along $\mathrm{w}^{q}$, as above.

(3) $p_{3}$ is purely stronger than $p_{2}$;

(4) $\quad p_{4} \leq p_{3}$ results from increasing halving parameters; and

(5) $q$ is the naturally defined "disjoint union" of $p_{4}$ and some condition $p^{\prime}$ which has the same $\mathrm{w}$ and halving parameters as $p_{4}, \operatorname{supp}\left(p^{\prime}\right)$ is disjoint from $\operatorname{supp}\left(p_{4}\right)$, and which jointly satisfies "modesty" with $p_{4}$.

Remark 3.5.5 - Every $q$ obtained by the above construction is stronger than $p$, provided it is a condition. Note that constructions (1), (2) and (5) always result in conditions (for (5), this is the same argument as in 2.5.8), whereas constructions (3) and (4) will generally decrease the norms of the compound creatures in an uncontrolled fashion. So to get a condition, we have to make sure that the norms of the new compound creatures still converge to infinity. Also, to be able to find a suitable $p^{\prime}$ in (5), we should make enough room for modesty in (3) (Fig. 3).

- The order is not entirely irrelevant, as gluing (2) has to be done before pure strengthening (3), since glued Sacks columns always have the form of products, whereas generally the Sacks columns in $q$ will not be of this form.

We will later use the following specific gluing construction.

Lemma 3.5.6 Assume that $\mathfrak{c}_{0}, \ldots, \mathfrak{c}_{n}$ is a properly stacked sequence of compound creatures, $n>0$, and $\operatorname{nor}\left(\mathfrak{c}_{i}\right) \geq M$ for all $i \leq n$. Pick for each $i<n$ some compound creatures $\mathfrak{d}_{i}$, purely stronger than $\mathfrak{c}_{i}$, such that $\mathfrak{d}_{i}$ and $\mathfrak{c}_{i}$ agree on the lim-inf part (but $\mathfrak{d}_{i}$ could consist of singletons on the lim-sup and the Sacks part). Set $\mathfrak{d}_{n}=\mathfrak{c}_{n}$. Then glue $\left(\mathfrak{d}_{0}, \ldots, \mathfrak{d}_{n}\right)$ has norm $\geq M$ as well.

Proof The lim-sup norm and the Sacks-norms will be large because nor $\left(\mathfrak{d}_{n}\right)=$ $\operatorname{nor}\left(\mathfrak{c}_{n}\right) \geq M$. The lim-inf norm will be large because we did not change anything on the lim-inf part.

\subsection{Projections and complete subforcings}

Lemma 3.6.1 Assume that $\Xi_{1 i} \subseteq \Xi^{\prime} \subseteq \Xi^{22}$ Let $\mathbb{Q} \Xi^{\prime} \subseteq \mathbb{Q}$ consist of all $p \in \mathbb{Q}$ with $\operatorname{supp}(p) \subseteq \Xi^{\prime}$. Then $\mathbb{Q} \Xi^{\prime}$ is a complete subforcing of $\mathbb{Q}$, and the restriction map is a projection on an open dense subset.

\footnotetext{
22 If we do not assume $\Xi^{\prime} \supseteq \Xi_{1 i}$ we get problems with the lim-inf norm when we combine the increased halving parameters of $q^{\prime}$ with the lim-inf creatures in $p_{1}$.
} 


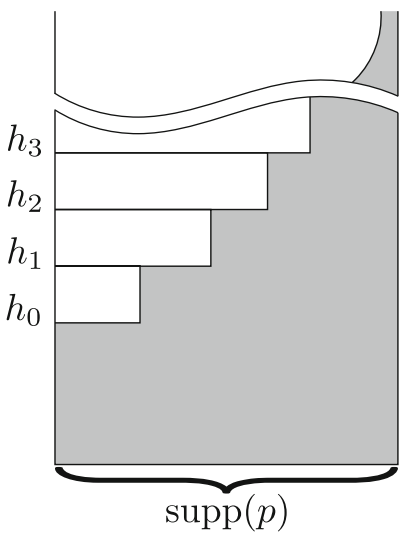

(a)

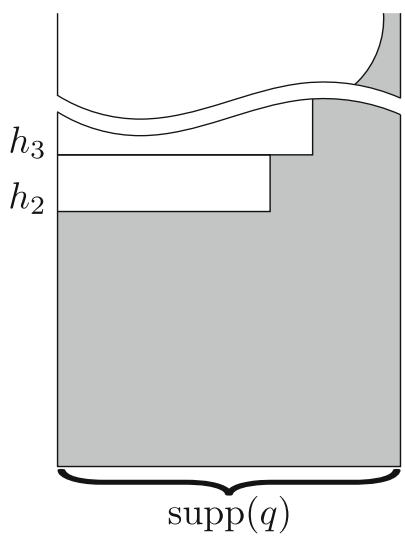

(b)

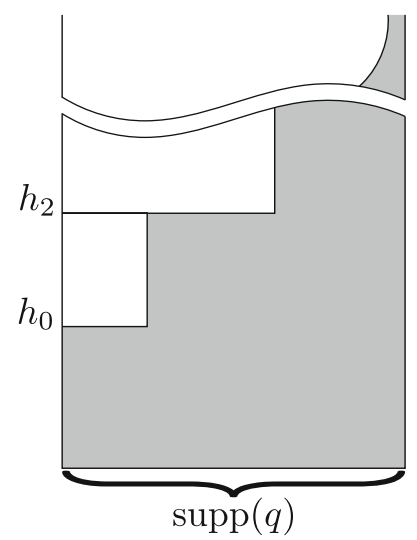

(c)

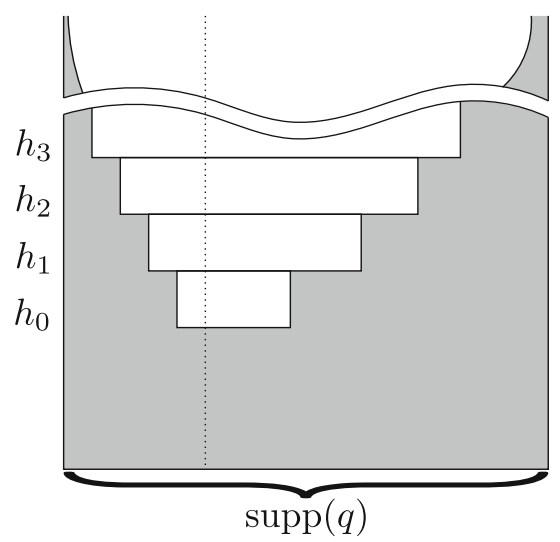

(d)

Fig. 3 a A schematic diagram of a condition $p$ of the forcing. The $h_{i}$ indicate an increasing enumeration of ${ }_{\mathrm{W}}{ }^{p}$, while the shaded region represents the domain of the trunk function $t^{p}$. $\mathbf{b}$ A condition $q=p \wedge \eta$, where $\eta \in \operatorname{poss}\left(p,<h_{2}\right)$. In particular, all of the compound creatures above level $h_{2}$ have been left unchanged, and the level below $h_{2}$ the condition $q$ consists entirely of trunk, with values determined by $\eta$. c A condition $q$ obtained from $p$ by gluing the pairs of compound creatures $p\left(h_{0}\right), p\left(h_{1}\right)$ and $p\left(h_{2}\right), p\left(h_{3}\right)$. Note that $\operatorname{trklg}^{q}(\eta)=h_{2}$ for any $\eta \in \operatorname{supp}(p)$ with $\operatorname{trklg} p(\eta)=h_{1}$ (and similarly if $\operatorname{trklg}^{p}(\eta)=h_{3}$ ). d A condition $q$ obtained as the "disjoint union" of $p$ and another condition (represented to the left of the dotted line) with the same $\mathrm{w}$ and the same halving parameters at each level as $p$

Of course, $\mathbb{Q}_{\Xi^{\prime}}$ will satisfy all the properties that we will prove generally for $\mathbb{Q}$ (as $\mathbb{Q} \Xi^{\prime}$ is defined just like $\mathbb{Q}$, only with a different underlying index set).

Proof The dense set $D$ is the set of all conditions $p$ with $\operatorname{supp}(p) \cap \Xi_{1 i} \neq \emptyset$. Fix $p \in D$, set $p^{\prime}:=p\left\lceil\Xi^{\prime}\right.$, and assume that $q^{\prime} \leq p^{\prime}$ is in $\mathbb{Q}^{\prime}$. It is enough to show that $q^{\prime}$ is compatible with $p$. To do this we will construct $q \leq p$ such that $q^{\prime}=q \uparrow \Xi^{\prime}$.

Set $p_{1}:=p \uparrow\left(\Xi \backslash \Xi^{\prime}\right)$. Increase the trunk of $p_{1}$ to $\min \left(\mathrm{w}^{q^{\prime}}\right)$, glue along $\mathrm{w}^{q^{\prime}}$, and increase the halving parameters to match those of $q^{\prime}$ to get a condition $q_{1} \leq p_{1}$ with 
$\mathrm{w}^{q_{1}}=\mathrm{w}^{q^{\prime}} \cdot{ }^{23}$ Letting $q$ be the disjoint union of $q_{1}$ and $q^{\prime}$, it follows that $q$ is a condition of $\mathbb{Q}$, and clearly $q \uparrow \Xi^{\prime}=q^{\prime}$.

\section{An inductive construction of $\mathbb{Q}$}

We will now review the "framework" from Definition 2.4.1, finally giving all the assumptions (including the previous Assumption 2.5.2) that are required to make the forcing proper.

In the following construction, we have the freedom to choose the following (as long as the assumptions are satisfied).

- $\Xi=\Xi_{1 \mathrm{~s}} \cup \Xi_{1 \mathrm{i}} \cup \Xi_{\mathrm{sk}}$, as in Definition 2.4.1.

- Natural numbers $H(<\mathbf{u})$ (for each sublevel $\mathbf{u}$ ) such that $H$ is increasing.

Remark. The function $H$ gives us the possibility to impose additional demands on the bigness $B$ (as given in (4.0.2), below). It is not needed to get properness and $\omega^{\omega}$-bounding, but will be used later ${ }^{24}$ in our specific constructions.

- For each $\xi \in \Xi_{\text {non-sk }}$ and each subatomic sublevel $\mathbf{u}$ the subatomic family $\mathrm{K}_{\xi, \mathbf{u}}$ living on some finite set $\operatorname{POSS}_{\xi, \mathbf{u}}$.

The other parameters are determined by the construction.

- Natural numbers maxposs $(<\mathbf{u})$ for each sublevel $\mathbf{u}$.

This will turn out to be an upper bound to the cardinality of $\operatorname{poss}(p,<\mathbf{u})$ for any pruned condition $p$.

- For each sublevel $\mathbf{u}$, we set

$$
B(\mathbf{u}):=2^{H(<\mathbf{u}) \cdot \operatorname{maxposs}(<\mathbf{u})}
$$

(and we set $B((0,-1)):=2) . B(\mathbf{u})$ is the bigness required for the subatoms (or Sacks columns) at $\mathbf{u}$.

- The Sacks intervals $I_{\mathrm{sk}, \ell}$ and subatomic index sets $J_{\ell}$, for each $\ell \in \omega$, as in Definition 2.4.1.

Note that, as usual, for a Sacks sublevel $\mathbf{u}=(\ell,-1)$ we may write $B(\ell)$ for $B(\mathbf{u})$. We similarly use maxposs $(<\ell)$ and $H(<\ell)$.

By induction of $\ell$ we now make the following definitions and requirements. (We also set the "initial values" $\operatorname{maxposs}(<(0,-1)):=1$ and $I_{\mathrm{sk},-1}=\{-1\}$.)

Basic Construction $(* 1)$ We require that $H(<\ell)>\operatorname{maxposs}(<\ell)+\ell+2$.

(*2) The Sacks sublevel. We let $I_{\mathrm{Sk}, \ell}$ be the interval starting at $\max \left(I_{\mathrm{Sk}, \ell-1}\right)+1$ and of minimal size such that $\operatorname{nor}_{\mathrm{Sacks}}^{B(\ell), \ell}\left(2^{I_{\mathrm{sk}, \ell}}\right) \geq \ell$.

This gives us "bigness" in the form of Lemma 2.3.6(6) for $B:=B(\ell)$.

\footnotetext{
23 As $\operatorname{supp}\left(p_{1}\right) \cap \Xi_{l i}=\emptyset$ it follows that increasing the halving parameters does not affect the norms of the compound creatures, and therefore $q_{1}$ is a condition of $\mathbb{Q}$.

24 Here is a very informal description of how $H$ will be used. The basic requirement is that at each sublevel $\mathbf{u}$ we have bigness (namely $B(\mathbf{u})$ ) which is large with respect to everything that happened below. However, the notion of "large with respect to" will slightly depend on the actual construction that increases the relevant cardinal characteristic. The parameter $H$ will allow us to accommodate these different interpretations. The function $H$ will be used as a parameter when defining "rapid reading" in Definition 5.1.1.
} 
$(* 3)$ We set $\operatorname{maxposs}(<(\ell, 0)):=\operatorname{maxposs}(<\ell) \cdot 2^{\left|I_{\mathrm{sk}, \ell}\right| \cdot \ell}$.

$(* 4)$ We set $J_{\ell}:=3^{(\ell+1) \cdot 2^{\ell \cdot \operatorname{maxposs}(<\ell)}}$. So $\mu^{\ell}\left(J_{\ell}\right)=2^{\ell \cdot \operatorname{maxposs}(<\ell)} \cdot 25$

(*5) The subatomic sublevels. By induction on $j \in J_{\ell}$ we now deal with the sublevel $\mathbf{u}=(\ell, j)$.

(a) For each $\xi \in \Xi_{\text {non-sk }}$, we require that $\mathrm{K}_{\xi, \mathbf{u}}$ is a subatomic family living on some finite set $\operatorname{POSS}_{\xi, \mathbf{u}}$.

(b) For each $\xi \in \Xi_{\text {non-sk }}$, we require that there is a subatom $x \in \mathrm{K}_{\xi \text {,u }}$ with norm at least $2^{\ell \cdot \operatorname{maxposs}(<\ell)}$

(c) For each $\xi \in \Xi_{\text {non-sk }}$, we require that $\mathrm{K}_{\xi, \mathbf{u}}$ is $B(\mathbf{u})$-big.

(d) We require that there is a uniform bound $M(\mathbf{u})=\max \left(\left\{\left|\operatorname{POSS}_{\xi, \mathbf{u}}\right|: \xi \in\right.\right.$ $\left.\left.\Xi_{\text {non-sk }}\right\}\right)$. Then we set, for $\mathbf{v}$ the successor sublevel of $\mathbf{u}$,

$$
\operatorname{maxposs}(<\mathbf{v}):=\operatorname{maxposs}(<\mathbf{u}) \cdot M(\mathbf{u})^{\ell+1} .
$$

(In particular this defines maxposs $(<(\ell+1,-1))$ if $\mathbf{u}=\left(\ell, J_{\ell}-1\right)$.)

The assumptions guarantee that the previous Assumption 2.5.2 is satisfied (in particular that there are compound creatures with norm $m^{\mathrm{dn}}$, and that $\mathbb{Q} \neq \varnothing$ ).

By induction, we immediately get the following (which is the reason for the name "maxposs").

Fact 4.0.3 Let $p$ be pruned. Then $|\operatorname{poss}(p,<\mathbf{u})| \leq \operatorname{maxposs}(<\mathbf{u})$ for $\mathbf{u} \in \operatorname{sblvls}(p)$. In particular, $|\operatorname{poss}(p,<h)| \leq \operatorname{maxposs}(<h)$ for $h \in \mathrm{w}^{p}$.

The following shows that each $p(\mathbf{u})$ is $B(\mathbf{u})$-big.

Fact 4.0.4 Let $p$ be a pruned condition, and let $\mathbf{u}$ be an active sublevel of $p$ (which can be Sacks or subatomic).

Then whenever $F: \operatorname{poss}^{\prime}(p,=\mathbf{u}) \rightarrow B(\mathbf{u})$ is a coloring, there is a strengthening $q(\mathbf{u})$ of $p(\mathbf{u})$ (i.e., either $q(\mathbf{u})$ is a subatom stronger than $p(\mathbf{u})$, or $q(\mathbf{u})$ is a sequence of Sacks columns such that each one is stronger than the according column in $p(\mathbf{u})$ ) such that the subatomic norm (or, each Sacks norm) decreases by at most 1 and such that $F\left\lceil\operatorname{poss}^{\prime}(q(\mathbf{u}))^{26}\right.$ is constant.

As $B(\mathbf{u})$ is much larger than maxposs $(<\mathbf{u})$, we also get a version of "compound bigness" (we will not directly use the following version, but we will use similar constructions). First note that a function $G: \operatorname{poss}^{\prime}(p, \leq \mathbf{u}) \rightarrow H(<\mathbf{u})$ may be interpreted as $F: \operatorname{poss}^{\prime}(p,=\mathbf{u}) \rightarrow H(<\mathbf{u})^{Y}$ for $Y:=\operatorname{poss}^{\prime}(p,<\mathbf{u})$ (cf. 2.7.4). As $\left|\operatorname{poss}^{\prime}(p,<\mathbf{u})\right| \leq \operatorname{maxposs}(<\mathbf{u})$, and $B(\mathbf{u})$ is big with respect to $\operatorname{maxposs}(<\mathbf{u})$ and $H(<\mathbf{u})$, we can use the previous item and strengthen $p(\mathbf{u})$ to make $G$ independent of the possibilities at $\mathbf{u}$.

\footnotetext{
$25 \mu^{\ell}$ is defined in 2.2.1.


from $q(\mathbf{u})$ if $\mathbf{u}$ is a Sacks sublevel.
} 
Iterating this downwards we get the following.

Fact 4.0.5 Let $p$ be pruned, and let $\mathbf{v}<\mathbf{u}$ be active sublevels of $p$.

- If $G: \operatorname{poss}^{\prime}(p,<\mathbf{u}) \rightarrow H(<\mathbf{v})$ is a coloring, then we can strengthen the $p\left(\mathbf{u}^{\prime}\right)$ to $q\left(\mathbf{u}^{\prime}\right)$ for $\mathbf{v} \leq \mathbf{u}^{\prime}<\mathbf{u}$, decreasing all subatomic/Sacks norms (and therefore also all compound norms) by at most 1 , such that $G$ restricted to $\operatorname{poss}^{\prime}(q,<\mathbf{u})$ only depends on $\operatorname{poss}^{\prime}(q,<\mathbf{v})$.

- In particular, if $G: \operatorname{poss}^{\prime}(p,<\mathbf{u}) \rightarrow 2$, then we can strengthen $p$ to $q$ as above such that $G\left\lceil\operatorname{poss}^{\prime}(q,<\mathbf{u})\right.$ is constant.

\section{Properness, $\omega^{\omega}$-bounding and rapid reading}

\subsection{Bigness, rapid reading from continuous reading}

(Remark: This section is the straight-forward modification of [12, Lemma 1.13].)

Definition 5.1.1 - Let $\underset{\sim}{\tau}$ be the name of an ordinal. We say that $\underset{\sim}{\tau}$ is decided below the sublevel $\mathbf{u}$ (with respect to the condition $p$ ), if $p \curlywedge \eta$ decides the value of $\underset{\sim}{\tau}$ for all $\eta \in \operatorname{poss}(p,<\mathbf{u})$; in other words, there is a function $R: \operatorname{poss}(p,<\mathbf{u}) \rightarrow \operatorname{Ord}$ such that $p \curlywedge \eta \Vdash \underset{\sim}{\tau}=R(\eta)$ for all $\eta \in \operatorname{poss}(p,<\mathbf{u})$.

- We also write " $\tau$ is decided $<\mathbf{u}$ "; and we write " $\tau$ is decided $\leq \mathbf{u}$ " for the obvious concept (i.e., " $\tau$ is decided $<\mathbf{v}$ ", where $\mathbf{v}$ is the successor sublevel of $\mathbf{u}$ ).

- $p$ essentially decides $\underset{\sim}{\tau}$, if there is some sublevel $\mathbf{u}$ such that $\tau$ is decided below $\mathbf{u}$.

- Let $\underset{\sim}{r}$ be the name of an $\omega$-sequence of ordinals. We say that a condition $p$ continuously reads $\underset{\sim}{r}$, if all $\underset{\sim}{r}(m)$ are essentially decided by $p$.

- $p$ rapidly reads $\underset{\sim}{r} \in 2^{\omega}$, if, for each sublevel $\mathbf{u}, \underset{\sim}{r} \uparrow H(<\mathbf{u})$ is decided below $\mathbf{u}$.

- Let $\Xi_{0} \subseteq \Xi$. We say that $p$ "reads $\underset{\sim}{r}$ continuously only using indices in $\Xi_{0}$ " if $p$ reads $\underset{\sim}{r}$ continuously and moreover (using the relevant functions $R$ mentioned above) the value of $R(\eta)$ depends only on $\eta \uparrow \Xi_{0}$.

In other words: For every $n$ there exists a sublevel $\mathbf{u}$ such that $p \curlywedge \eta$ decides the value of $\underset{\sim}{r}(n)$ for all $\eta \in \operatorname{poss}(p,<\mathbf{u})$, and whenever $\eta \uparrow \Xi_{0}=\eta^{\prime} \uparrow \Xi_{0}$, then $p \curlywedge \eta$ and $p \curlywedge \tilde{\eta}^{\prime}$ agree on the value of $\underset{\sim}{r}(n)$.

- We define the notion "reads $\underset{\sim}{r}$ rapidly only using indices in $\Xi_{0}$ " similarly.

- Instead of "only using indices in $\Xi \backslash \Xi_{1}$ " we also write "not using indices in $\Xi_{1}$ ".

Note that for $X \supseteq \Xi_{l i}$, a real $\underset{\sim}{r}$ is read continuously from $X$ iff it exists in the $\mathbb{Q}_{X}$-extension (cf. 3.6.1).

Remark 5.1.2 For a fixed condition $p$, the possibilities (at all sublevels) form an infinite tree in the obvious way. The set of branches $T_{p}$ of this tree carries a natural topology. $p$ continuously reads $\tau$ iff there is a continuous function $F$ on $T_{p}$ in the ground model such that $p$ forces $\underset{\sim}{\tau}=\tilde{F}(\bar{y})$, where $\tilde{F}$ is the canonical extension of $F$.

In our case, the tree is finitely splitting, so $T_{p}$ is compact, and continuous is the same as uniformly continuous. (Note that the definition above really uses a uniform notion of continuity.)

Rapid reading corresponds to a form of Lipschitz continuity. 
Lemma 5.1.3 (1) If $p$ continuously (or: rapidly) reads $\underset{\sim}{r}$ and $q \leq p$ with $\operatorname{supp}(q) \supseteq$ $\operatorname{supp}(p)$, then $q$ continuously (or: rapidly) reads $\underset{\sim}{\sim}$. The same holds if we add “only using $\Xi_{0}$ ” or: "not using $\Xi_{1}$ ”.

(2) If $q \leq \leq^{*} p$, and $\tau$ is a name of an ordinal essentially decided by $p$, then also $q$ essentially decides $\tau$.

Proof (1) Intuitively, this is clear: If $q \leq p$ and $\eta \in \operatorname{poss}(q,<\mathbf{u})$ then $\eta$ morally is an element of $\operatorname{poss}(p,<\mathbf{u})$, and $q \curlywedge \eta \leq p \curlywedge \eta$.

The formal proof uses Lemma 3.2.1.

(2) $\quad p$ forces that $\underset{\sim}{\tau}$ is decided by a finite case distinction; so $q$ forces the same.

Lemma 5.1.4 In $V$, let $\kappa$ be $\max \left(\aleph_{0},\left|\Xi_{0}\right|\right)^{\aleph_{0}}$. Then in the extension, there are at most $\kappa$ many reals which are continuously read only using ${ }^{27}$ indices in $\Xi_{0}$.

Proof This is the usual "nice names" argument: Given $p$ continuously reading $\underset{\sim}{r}$. We can define the obvious name $\underset{\sim}{r^{\prime}}$ continuously read by $p^{\prime}=p\left\lceil\Xi_{0}\right.$, such that $p$ forces $\underset{r}{r}={\underset{\sim}{r}}^{\prime}$. There are at most $\kappa$ many countable subsets of $\Xi_{0}$, and therefore only $\kappa$ many conditions $p^{\prime}$ with $\operatorname{supp}\left(p^{\prime}\right) \subseteq \Xi_{0}$. Given such a condition $p^{\prime}$, there are only $2^{\aleph_{0}}$ many ways to continuously read a real (with respect to $p^{\prime}$ ).

We will first show that we can "densely" get from continuous reading to rapid reading. Later we will show that "densely" we can continuously read reals. Both proofs are the obvious modifications of the corresponding proofs in [12].

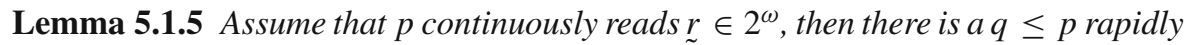
reading $\underset{\sim}{r}$.

The same is true if we add "only using $\Xi_{0}$ ".

Proof Without loss of generality we can assume that $p$ is pruned (use Lemmas 3.4.3 and 5.1.3).

For a sublevel $\mathbf{u}$, we set

$\mathbf{v}^{\operatorname{dec}}(\mathbf{u})$ is the maximal sublevel such that $\underset{\sim}{r} \uparrow H\left(<\mathbf{v}^{\operatorname{dec}}(\mathbf{u})\right)$ is decided below $\mathbf{u}$,

The function $\mathbf{v}^{\mathrm{dec}}$ is nondecreasing; and continuous reading implies that $\mathbf{v}^{\mathrm{dec}}$ is an unbounded function on the sublevels; but $\mathbf{v}^{\text {dec }}$ can generally grow very slowly. ( $p$

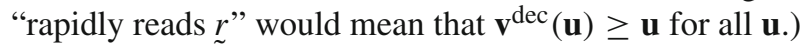

For all sublevels $\mathbf{v} \leq \mathbf{u}$ we set



There are at most

$$
2^{H(<\mathbf{v})}
$$

many possibilities for $\underset{\sim}{\mathbf{u}}$, as $H\left(\left(<\min \left(\mathbf{v}, \mathbf{v}^{\operatorname{dec}}(\mathbf{u})\right)\right)\right) \leq H(<\mathbf{v})$.

\footnotetext{
27 More formally: reals $r$ such that there is a $p \in G$ and a name $\underset{\sim}{r}$ such that $p$ continuously reads $\underset{\sim}{r}$ only using $\Xi_{0}$ and such that $G$ evaluates $\underset{\sim}{r}$ to $r$.
} 
1: For now, fix a Sacks sublevel $\mathbf{u}=(\ell,-1)$ with $\ell \in \mathrm{w}^{p}$.

We will define (or rather: pick) by downwards induction on $\mathbf{u}^{\prime} \in \operatorname{sblvls}(p), \mathbf{u}^{\prime} \leq \mathbf{u}$, objects $\mathfrak{d}_{\mathbf{u}^{\prime}}^{\mathbf{u}}$, which are either a sequence of Sacks columns (if $\mathbf{u}^{\prime}$ is Sacks) or a subatom; and functions $\psi_{\mathbf{u}^{\prime}}^{\mathbf{u}}$.

1a: For $\mathbf{u}^{\prime}=\mathbf{u}$, we set $\mathfrak{d}_{\mathbf{u}}^{\mathbf{u}}:=p(\mathbf{u})$, i.e., the sequence of Sacks columns of level $\ell$. We let $\psi_{\mathbf{u}}^{\mathbf{u}}$ be the function with domain $\operatorname{poss}(p,<\mathbf{u})$ which assigns to each $\eta \in$ $\operatorname{poss}(p,<\mathbf{u})$ the corresponding value of $\underset{\sim}{x_{\mathbf{u}}^{\mathbf{u}}}$.

In other words: $p \curlywedge \eta$ forces that $\underset{\sim}{\mathbf{u}}=\psi_{\mathbf{u}}^{\mathbf{u}}(\eta)$ for each $\eta \in \operatorname{poss}(p,<\mathbf{u})$.

1b: We continue the induction on $\mathbf{u}^{\prime}$. For now, we write $\mathfrak{d}^{\prime}:=\mathfrak{d}_{\mathbf{u}^{\prime}}^{\mathbf{u}}, \psi^{\prime}:=\psi_{\mathbf{u}^{\prime}}^{\mathbf{u}}$, and $x^{\prime}:=x_{\mathbf{u}^{\prime}}^{\mathbf{u}}$.

- If $\mathbf{u}^{\prime}$ is subatomic, then we choose for $\mathfrak{d}^{\prime}$ a subatom stronger than the active subatom $p\left(\mathbf{u}^{\prime}\right)$, with nor $\left(\mathfrak{d}^{\prime}\right) \geq \operatorname{nor}\left(p\left(\mathbf{u}^{\prime}\right)\right)-1$.

- Otherwise, i.e., if $\mathbf{u}^{\prime}=\left(\ell^{\prime},-1\right)$ is Sacks with $\ell^{\prime} \in \mathrm{w}^{p}$, set $S:=\operatorname{supp}\left(p, \ell^{\prime}\right) \cap$ $\Xi_{\mathrm{Sk}} \neq \emptyset$. Then $\mathfrak{d}^{\prime}$ is a sequence $\left(\mathfrak{s}_{\xi}^{\prime}\right)_{\xi \in S}$ of Sacks columns such that $\mathfrak{s}_{\xi}^{\prime} \subseteq p\left(\xi, \ell^{\prime}\right)$ and $\operatorname{nor}_{\text {Sacks }}\left(\mathfrak{s}_{\xi}^{\prime}\right) \geq \operatorname{nor}_{\text {Sacks }}(p(\xi, \ell))-1$ for each $\xi \in S$.

- $\psi^{\prime}$ is a function with domain $\operatorname{poss}\left(p,<\mathbf{u}^{\prime}\right) \operatorname{such}$ that

modulo $\left(\mathbf{v}: \mathbf{u}^{\prime} \leq \mathbf{v}<\mathbf{u}\right)$, each $\eta \in \operatorname{poss}\left(p,<\mathbf{u}^{\prime}\right)$ decides $\underset{\sim}{x^{\prime}}$ to be $\psi^{\prime}(\eta)$

by which we mean:

$p \curlywedge \eta$ forces the following: If the generic $\bar{y}$ is compatible with $\mathfrak{d}_{\mathbf{v}}^{\mathbf{u}}$ for each sublevel $\mathbf{v} \in \operatorname{sblvls}(p)$ with $\mathbf{u}^{\prime} \leq \mathbf{v} \underset{<}{<} \mathbf{u}$, then $\underset{\sim}{x^{\prime}}=\psi^{\prime}(\eta)$.

How can we find such $\mathfrak{d}^{\prime}, \psi^{\prime}$ ?

Let $\mathbf{u}^{\prime \prime}$ be the smallest element of $\operatorname{sblvls}(p)$ above $\mathbf{u}^{\prime}$. By induction we already know that $\psi^{\prime \prime}:=\psi_{\mathbf{u}^{\prime \prime}}^{\mathbf{u}}$ is a function with domain $\operatorname{poss}\left(p,<\mathbf{u}^{\prime \prime}\right)$ such that modulo



Let $\psi_{0}^{\prime \prime}(\eta)$ be the restriction of $\psi^{\prime \prime}(\eta)$ to $H\left(<\tilde{\min }\left(\mathbf{u}^{\prime}, \mathbf{v}^{\operatorname{dec}}(\mathbf{u})\right)\right)$, i.e., $\psi_{0}^{\prime \prime}$ maps each $\eta \in \operatorname{poss}\left(p,<\mathbf{u}^{\prime \prime}\right)$ to a restriction of $\underset{\sim}{x \prime}$, which is a potential value for $\underset{\sim}{x^{\prime}}$.

We can write ${ }^{28} \psi_{0}^{\prime \prime}$ as a function $A \times B \rightarrow C$, for $A:=\tilde{\operatorname{poss}}\left(p,<\mathbf{u}^{\prime}\right)$, $B=\operatorname{poss}\left(p,=\mathbf{u}^{\prime}\right)$ and $C$ is the set of possible values of $\underset{\sim}{x^{\prime}}$, which has, according to (5.1.8), size $\leq 2^{H\left(<\mathbf{u}^{\prime}\right)}$. This defines a function from $B$ to $C^{A}$, a set of cardinality $\leq 2^{\operatorname{maxposs}\left(<\mathbf{u}^{\prime}\right) \cdot H\left(<\mathbf{u}^{\prime}\right)}$; so according to (4.0.2) and Fact 4.0.4 we can use bigness at sublevel $\mathbf{u}^{\prime}$ to find $\mathfrak{d}^{\prime}$ such that $\psi_{0}^{\prime \prime}$ does not depend on sublevel $\mathbf{u}^{\prime}$. This naturally defines $\psi^{\prime}$.

2: We perform this downwards induction from each Sacks sublevel $\mathbf{u}$ of $p$. So this defines for each $\mathbf{v}<\mathbf{u}$ in $\operatorname{sblvls}(p)$ the objects $\mathfrak{d}_{\mathbf{v}}^{\mathbf{u}}$ and $\psi_{\mathbf{v}}^{\mathbf{u}}$, satisfying (which is just 5.1.9):

28 cf. 2.7.4. 
modulo $\left(\mathbf{v}^{\prime}: \mathbf{v} \leq \mathbf{v}^{\prime} \leq \mathbf{u}\right)$, each $\eta \in \operatorname{poss}(p,<\mathbf{v})$ decides $\underset{\sim}{\mathbf{u}}$ to be $\psi_{\mathbf{v}}^{\mathbf{u}}(\eta)$

Also, the norms of each Sacks column and subatom drop by at most 1 .

3: Note that for a given $\mathbf{v}$, there are only finitely many possibilities for $\mathfrak{d}_{\mathbf{v}}^{\mathbf{u}}$ and $\psi_{\mathbf{v}}^{\mathbf{u}}$. So by König's Lemma there is a sequence $\left(\mathfrak{d}_{\mathbf{v}}^{*}, \psi_{\mathbf{v}}^{*}\right)_{\mathbf{v} \in \operatorname{sblvls}(p)}$ such that

for each sublevel $\mathbf{v}^{\prime}$ there is an $\mathbf{u}>\mathbf{v}^{\prime}$ such that $\mathfrak{d}_{\mathbf{v}^{\prime \prime}}^{\mathbf{u}}=\mathfrak{d}_{\mathbf{v}^{\prime \prime}}^{*}$ and $\psi_{\mathbf{v}^{\prime \prime}}^{\mathbf{u}}=\psi_{\mathbf{v}^{\prime \prime}}^{*}$ for all $\mathbf{v}^{\prime \prime} \leq \mathbf{v}^{\prime}$.

4: We now construct $q$ by replacing the subatoms and Sacks columns in $p$ at sublevel $\mathbf{v}$ with $\mathfrak{d}_{\mathbf{v}}^{*}$ (for each $\mathbf{v} \in \operatorname{sblvls}(p)$ ). So $q$ has the same $\mathrm{w}$ as $p$, the same supports, the same halving parameters and the same trunk; and all norms decrease by at most 1 . We claim that $q$ rapidly reads $\underset{\sim}{r}$, i.e., we claim that each $\eta \in \operatorname{poss}(q,<\mathbf{v})$ decides $\underset{\sim}{r} \uparrow H(<\mathbf{v})$.

5: Pick a $\mathbf{v}^{\prime}>\mathbf{v}$ such that $\mathbf{v}^{\operatorname{dec}}\left(\mathbf{v}^{\prime}\right) \geq \mathbf{v}$. According to the definition (5.1.6), this means that $\underset{\sim}{r} \uparrow H(<\mathbf{v})$ is decided below $\mathbf{v}^{\prime}$. Then pick $\mathbf{u}>\mathbf{v}^{\prime}$ as in (5.1.11). Recall (from (5.1.10)) that $\underset{\sim}{\mathbf{u}} \mathbf{u}$ is decided below $\mathbf{v}$ by $\psi_{\mathbf{v}}^{\mathbf{u}}$ modulo the sequence $\left(\mathfrak{d}_{\mathbf{v}^{\prime \prime}}^{\mathbf{u}}: \mathbf{v} \leq\right.$ $\left.\mathbf{v}^{\prime \prime}<\mathbf{u}\right)$. Recall that $\mathbf{v}^{\operatorname{dec}}\left(\mathbf{v}^{\prime}\right) \geq \mathbf{v}$ and $\mathbf{u} \geq \mathbf{v}^{\prime}$. So $\min \left(\mathbf{v}^{\operatorname{dec}}(\mathbf{u}), \mathbf{v}\right)=\mathbf{v}$, therefore $\underset{\sim}{\mathbf{v}_{\mathbf{v}}^{\mathbf{u}}}=\underset{\sim}{r}\left\lceil H(<\mathbf{v})\right.$. And, since $\mathbf{v}^{\mathrm{dec}}\left(\mathbf{v}^{\prime}\right) \geq \mathbf{v},{\underset{\sim}{\mathbf{v}}}_{\mathbf{v}}^{\mathbf{u}}$ is decided already (by the original condition $p$ ) below $\mathbf{v}^{\prime}$. So we can omit the assumption that the generic is compatible with $\mathfrak{d}_{\mathbf{u}^{\prime \prime}}^{\mathbf{u}}$ for any $\mathbf{v}^{\prime} \leq \mathbf{u}^{\prime \prime}<\mathbf{u}$ and still correctly compute $\underset{\sim}{x} \underset{\mathbf{v}}{\mathbf{u}}$ with $\psi_{\mathbf{v}}^{\mathbf{u}}$ modulo $\left(\mathfrak{d}_{\mathbf{u}^{\prime \prime}}^{\mathbf{u}}: \mathbf{v} \leq \mathbf{u}^{\prime \prime}<\mathbf{v}^{\prime}\right)$.

In particular, $\psi_{\mathbf{v}}^{\mathbf{u}}=\psi_{\mathbf{v}}^{*}$ correctly computes $\underset{\sim}{\mathbf{v}} \mathbf{u}=\underset{\sim}{r} \uparrow H(<\mathbf{v})$ modulo $q$ (since $q$ contains $\mathfrak{d}_{\mathbf{u}^{\prime \prime}}^{\mathbf{u}}=\mathfrak{d}_{\mathbf{u}^{\prime \prime}}^{*}$ for each $\mathbf{u}^{\prime \prime}<\mathbf{v}^{\prime}$.)

\subsection{Halving and unhalving}

We will now, for the first and only time in this paper, make use of the halving parameter. We will show how to "halve" a condition $q$ to half $(q)$, and then "unhalve" any $r \leq$ half $(q)$ with "positive norms" to some $s \leq^{*} q$ with "large norms". This fact will only be used in the next section, to show pure decision.

We repeat the definition of the lim-inf norm from 2.5.3:

$$
\begin{aligned}
& \operatorname{nor}_{\liminf }^{\operatorname{maxposs}\left(<m^{\mathrm{dn}}\right)}(\mathfrak{c}, h)=\frac{\log _{2}\left(N_{h}^{\mathfrak{c}}-d(\mathfrak{c}, h)\right)}{\operatorname{maxposs}\left(<m^{\mathrm{dn}}\right)} \\
& \quad \text { for } N_{h}^{\mathfrak{c}}:=\min \left\{\operatorname{nor}(\mathfrak{c}(\xi, h)): \xi \in \operatorname{supp} \cap \Xi_{l_{i}}\right\} .
\end{aligned}
$$

If we increase $d:=d(\mathfrak{c}, h)$ to

$$
d^{\prime}:=d+\frac{N_{h}^{\mathfrak{c}}-d}{2}=\frac{N_{h}^{\mathfrak{c}}+d}{2},
$$

then the resulting lim-inf norm (hence also the compound norm) decreases by at most $1 / \operatorname{maxposs}\left(<m^{\mathrm{dn}}\right)$. 
Definition 5.2.2 Given a compound creature $\mathfrak{c}$, we set half $(\mathfrak{c})$ to be the same compound creature as $\mathfrak{c}$, except that we replace each halving parameter $d(h)$ by the $d^{\prime}(h)$ described above.

So nor $($ half $(\mathfrak{c})) \geq \operatorname{nor}(\mathfrak{c})-1 / \operatorname{maxposs}\left(<m^{\mathrm{dn}}\right)$.

Similarly, given a condition $p$ and a level $h \in \mathrm{w}^{p}$, we set half $(p, \geq h)$ to be the same as $p$, except that all compound creatures $p(\ell)$ for $\ell \geq h$ are halved (and nothing changes below $h$ ).

The point of halving is the following: Assume that the norms in $q$ are "large" and that $r \leq$ half $(q)$ has norms that are just $>0$. Then there is an "unhalved version" of $r$, an $s \leq q$, such that the norms in $s$ are "large" and still $s \leq * r$.

In more detail:

\section{Lemma 5.2.3 (Unhalving) Fix}

- $M \in \mathbb{R}$,

- a condition $q$,

- $h \in \mathrm{w}^{q}$ such that $\operatorname{nor}(q(\ell)) \geq M$ for all $\ell \geq h$ in $\mathrm{w}^{q}$,

- a condition $r \leq$ half $(q, \geq h)$ such that $\min \left(\mathrm{w}^{r}\right)=h$ and $\operatorname{nor}(r(\ell))>0$ for all $\ell$ in $\mathrm{w}^{r}$.

Then there is an such that

(1) $s \leq q$.

(2) $h=\min \left(\mathrm{w}^{s}\right)$.

(3) Writing $h_{1}$ for the successor of $h$ in $\mathrm{w}^{s}$, we have $\operatorname{nor}(s, \ell) \geq M$ for all $\ell \geq h_{1}$ in $\mathrm{w}^{\mathrm{s}}$.

(4) $\operatorname{supp}(s, h)=\operatorname{supp}(q, h)$.

(5) Above $h_{1}, s$ is the same as $r$, i.e.:

- For $\ell \geq h_{1}: \ell \in \mathrm{w}^{s}$ iff $\ell \in \mathrm{w}^{r}$, and for such $\ell$ we have $s(\ell)=r(\ell)$.

- The trunks agree above $h_{1}$.

- So in particular, $\operatorname{supp}(s)=\operatorname{supp}(r)$, and the norms do not change above $h_{1}$ (hence are $\geq M$ ).

(6) $\operatorname{nor}(s, h) \geq M-1 / \operatorname{maxposs}(<h)$.

(7) $\operatorname{poss}\left(s,<h_{1}\right) \subseteq \operatorname{poss}\left(r,<h_{1}\right)$.

Note that (5) and (7) implies $s \leq^{*} r$ (by 3.1.2). So (by 5.1.3), if $r$ essentially decides a name $\underset{\sim}{\tau}$, then so does $s$.

Proof First fix $h_{0} \in \mathrm{w}^{r}$ bigger than $h$ such that $\operatorname{nor}(r(\ell))>M$ for all $\ell \geq h_{0}$. Let $h_{1}$ be the $\mathrm{w}^{r}$-successor of $h_{0}$.

We set $\mathrm{w}^{s}:=\{h\} \cup \mathrm{w}^{r} \backslash h_{1}$. The trunk $t^{s}$ will extend $t^{r}$ (and will contain some additional information in the "area" $\left.\left[h, h_{1}\right) \times\left(\operatorname{supp}\left(r, h_{0}\right) \backslash \operatorname{supp}(q, h)\right)\right)$.

For $\ell \geq h_{1}$ in $\mathrm{w}^{s}$, we set $s(\ell):=r(\ell)$.

We set $\mathfrak{d}_{0}:=\operatorname{glue}\left(r(h), \ldots, r\left(h_{0}\right)\right)$, and choose arbitrary $r$-compatible elements for the new parts of the trunk $t^{s}$. We then let $\mathfrak{d}_{1}$ be the restriction of $\mathfrak{d}_{0}$ to $\operatorname{supp}(q, h)$ (again, choosing $r$-compatible elements for the new parts of the trunk $t^{s}$ ).

Now we construct $\mathfrak{d}$ from $\mathfrak{d}_{1}$ by replacing each halving parameter $d^{\mathfrak{d}_{1}}(k)$ by $d^{q}(k)$ (for all $h \leq k<h_{1}$ ). We set $s(h)=\mathfrak{d}$. This completes the construction of the condition $s$.

It is straightforward to check that the requirements are satisfied. We will show $\operatorname{nor}(s(h))=\operatorname{nor}(\mathfrak{d}) \geq M-1 / \operatorname{maxposs}(<h)$ : 
The norm of $\mathfrak{d}$ is the minimum of several subnorms:

- The width norm, which is $\geq M$, as $\operatorname{supp}(\mathfrak{d})=\operatorname{supp}(q, h)$ and $\operatorname{nor}(q(h)) \geq M$.

- The Sacks norms of the Sacks columns $\mathfrak{d}(\xi)=r(\xi, h) \otimes \cdots \otimes r\left(\xi, h_{0}\right)$ for $\xi \in \operatorname{supp}(\mathfrak{d}) \cap \Xi_{\mathrm{sk}}$ :

$$
\begin{aligned}
\operatorname{nor}_{\text {Sacks }}(\mathfrak{d}(\xi)) & =\operatorname{nor}_{\mathrm{Sacks}}^{B(h), h}(\mathfrak{d}(\xi)) \geq \operatorname{nor}_{\mathrm{Sacks}}^{B(h), h}\left(r\left(\xi, h_{0}\right)\right) \\
& \geq \operatorname{nor}_{\mathrm{Sacks}}^{B\left(h_{0}\right), h_{0}}\left(r\left(\xi, h_{0}\right)\right)=\operatorname{nor}_{\text {Sacks }}\left(r\left(\xi, h_{0}\right)\right) \geq M,
\end{aligned}
$$

by 2.3.6.

- The lim-sup norms: $\operatorname{nor}_{\text {limsup }}(\mathfrak{d}, \xi) \geq \operatorname{nor}_{\text {limsup }}\left(r\left(h_{0}\right), \xi\right) \geq M$.

- So it remains to deal with the lim-inf norm.

So we have to show that for $h \leq \ell<h_{1}$,

$$
\operatorname{nor}_{\liminf }^{\operatorname{maxposs}(<h)}(\mathfrak{d}, \ell)=\frac{\log _{2}\left(N_{\ell}^{\mathfrak{d}}-d(\mathfrak{c}, \ell)\right)}{\operatorname{maxposs}(<h)} \geq M-\frac{1}{\operatorname{maxposs}(<h)},
$$

where $N_{\ell}^{\mathfrak{d}}:=\min \left\{\operatorname{nor}(\mathfrak{d}(\xi, \ell)): \xi \in \operatorname{supp}(\mathfrak{d}) \cap \Xi_{l i}\right\}$.

Recall $d^{\prime}(\ell)$ as defined in (5.2.1). These are the halving parameters used in half $(q)$, and since $r \leq$ half $(q)$ we know that $d^{r}(\ell) \geq d^{\prime}(\ell)$ (where $d^{r}$ are the halving parameters used in $r$ ).

Let $m \in \mathrm{w}^{r}$ correspond to $\ell$ (i.e., $m \leq \ell$ and $\ell$ less than the $\mathrm{w}^{r}$-successor of $m$ ). As $\operatorname{nor}(r(m))>0$, we know that

$$
0<\operatorname{nor}_{\liminf }^{\operatorname{maxposs}(<m)}(r(m), \ell) \leq \operatorname{nor}_{\liminf }^{\operatorname{maxposs}(<h)}(r(m), \ell) \leq \frac{\log _{2}\left(N_{\ell}^{\mathfrak{d}}-d^{r}(\ell)\right)}{\operatorname{maxposs}(<h)}
$$

for $N_{\ell}^{\mathfrak{d}}$ as above. ${ }^{29}$

Fix any $\xi \in \operatorname{supp}(q, h) \cap \Xi_{1 i}$. Let $k \in \mathrm{w}^{q}$ correspond to $\ell$ (as above), and set $\mathfrak{c}=q(k)$. The inequality above gives $0<\log _{2}\left(\operatorname{nor}(\mathfrak{d}(\ell, \xi))-d^{r}(\ell)\right)$, which implies

$$
\operatorname{nor}(\mathfrak{o}(\xi, \ell))>d^{r}(\ell) \geq d^{\prime}(\ell)=d^{q}(\ell)+\frac{N_{\ell}^{\mathfrak{c}}-d^{q}(\ell)}{2} .
$$

So nor $(\mathfrak{d}(\ell, \xi))-d^{q}(\ell)>\frac{N_{\ell}^{\mathfrak{c}}-d^{q}(\ell)}{2}$ for all $\xi$, and so

$$
\begin{aligned}
\text { nor }_{\liminf }^{\operatorname{maxposs}(<h)}(\mathfrak{d}, \ell) & \geq \text { nor }_{\liminf }^{\operatorname{maxposs}(<h)}(\mathfrak{c}, \ell)-\frac{1}{\operatorname{maxposs}(<h)} \\
& \geq \operatorname{nor}_{\liminf }^{\operatorname{maxposs}(<k)}(\mathfrak{c}, \ell)-\frac{1}{\operatorname{maxposs}(<h)} \\
& \geq M-\frac{1}{\operatorname{maxposs}(<h)} .
\end{aligned}
$$

\footnotetext{
29 The last $\leq$ holds since $r(m)$ contains the same subatoms as $\mathfrak{d}$ (on the common support; however the support of $r(m)$ may be larger, therefore the last inequality is not necessarily an equality).
} 


\subsection{Halving and pure decision}

(Remark: This section is a straightforward modification of [12, Lemma 1.17].)

Lemma 5.3.1 Suppose that $\tau$ is a name for an element of $V$, that $p_{0} \in \mathbb{Q}$, that $M_{0} \in \mathrm{w}^{p_{0}}$ and $n_{0} \geq 1$ are such that $\operatorname{nor}\left(p_{0}(h)\right) \geq n_{0}+2$ for all $h \in \mathrm{w}^{p_{0}} \backslash M_{0}$. Then there is a condition $q$ such that:

- $q \leq p_{0}$.

- $q$ essentially decides $\tau$.

- Below $M_{0}, q$ and $p_{0}$ are identical, ${ }^{30}$ i.e.: $\mathrm{w}^{q} \cap M_{0}={ }_{\mathrm{w}}{ }^{p_{0}} \cap M_{0}$ and $q(h)=p_{0}(h)$ for all $h \in \mathrm{w}^{q} \cap M_{0}$.

- $\operatorname{nor}(q(h)) \geq n_{0}$ for all $h \in \mathrm{w}^{q} \backslash M_{0}$.

Proof We may assume that $p_{0}$ is pruned. Our proof will consist of several steps:

1. Using halving; the mini-steps.

Suppose that we are given $p \in \mathbb{Q}, M \in \mathrm{w}^{p}$, and $n \geq 1$ such that nor $(p(h))>n$ for all $h \in \mathrm{w}^{p} \backslash M$. We show how to construct an extension of $p$, denoted $r(p, M, n)$.

First enumerate $\operatorname{poss}(p,<M)$ as $\left(\eta^{1}, \ldots, \eta^{m}\right)$. Note that $m \leq \operatorname{maxposs}(<M)$. Setting $p^{0}=p$, we inductively construct conditions $p^{1}, \ldots, p^{m}$ and the auxiliary conditions $\tilde{p}^{1}, \ldots, \tilde{p}^{m}$ so that for each $k<m$ the following holds:

(1) $\tilde{p}^{k+1}$ is $p^{k}$ where we replace everything below $M$ (and in $\operatorname{supp}(p)$ ) with $\eta^{k+1}$. Remarks:

- By (3) below, we will get $\min \left(\mathrm{w}^{\tilde{p}^{k+1}}\right)=M$.

- If $k=0$, then $\tilde{p}^{1}$ is just $p \wedge \eta^{1}$. But for $k>0, \eta^{k+1}$ will not be in $\operatorname{poss}\left(p^{k},<M\right)$, so we cannot use the notation $\tilde{p}^{k+1}=p^{k} \wedge \eta^{k+1}$.

- Note that generally $\operatorname{supp}\left(p^{k}\right)$ will be $\operatorname{larger}$ than $\operatorname{supp}(p)$, so we do not replace the whole trunk below $M$ by $\eta^{k+1}$, but just the part in $\operatorname{supp}(p)$.

(2) $p^{k+1} \leq \tilde{p}^{k+1}$. Note that we do not have $p_{k+1} \leq p_{k}$, for trivial reasons: their trunks are incompatible.

(3) $\min \left(\mathrm{w}^{p^{k+1}}\right)=M$.

Remarks:

- So by strengthening $\tilde{p}^{k+1}$ to $p^{k+1}$, we do not increase the overall trunk-length $\min (\mathrm{w})$.

- Note that we do not assume that $\mathrm{w}^{p^{k+1}}=\mathrm{w}^{p^{k}} \backslash M$, i.e., generally the $\mathrm{w}$-sets will become thinner due to gluing.

(4) $\operatorname{supp}\left(p^{k+1}, M\right)=\operatorname{supp}(p, M)$.

- Remark: This only holds at level $M$ : Generally, $\operatorname{supp}\left(p^{k+1}\right)$ will be larger than $\operatorname{supp}\left(p^{k}\right)$.

(5) $\operatorname{nor}\left(p^{k+1}, h\right)>n-\frac{k+1}{\operatorname{maxposs}(<M)}$ for all $h \in \mathrm{w}^{p^{k+1}} \backslash M$.

(6) One of the following two cases holds:

- (decide) $p^{k+1}$ essentially decides $\tau$.

- (halve) $p^{k+1}=\operatorname{half}\left(\tilde{p}^{k+1}, \geq M\right)$.

More explicitly: If the deciding case is possible, then we use it. Only if it is not possible, we halve.

\footnotetext{
$30 \operatorname{supp}(q)$ can be larger than $\operatorname{supp}(p)$, so below $M_{0}$ there will be new parts of the trunk $t^{q}$.
} 
We then define $r=r(p, M, n)$ as follows: Below $M, r$ is identical to $p$; and above (including) $M, r$ is identical to $p^{m}$ (the last one of the $p^{k}$ constructed above). In more detail:

- $\mathrm{w}^{r}=\left(\mathrm{w}^{p} \cap M\right) \cup\left(\mathrm{w}^{p^{m}} \backslash M\right)$; i.e., below $M$ the levels of $r$ are the ones of $p$; and above (including) $M$ the levels of $r$ are the ones of $p^{m}$.

- $r(h)=p(h)$ for all $h \in \mathrm{w}^{r} \cap M$;

- $r(h)=p^{m}(h)$ for all $h \in \mathrm{w}^{r} \backslash M$;

- This determines the domain of $t^{r}$; and we set $t^{r}$ to be $t^{p^{m}}$ restricted to this domain. $r=r(p, M, n)$ has the following properties:

- $r \in \mathbb{Q}, r \leq p$.

- $\operatorname{nor}(r(\ell))>n-1$ for all $\ell \geq M$ in $\mathrm{w}^{r}$.

- If $\eta \in \operatorname{poss}(r,<M)$ and if there is a $s \leq r \wedge \eta$ such that $s$ essentially decides $\underset{\sim}{\tau}, \min \left(\mathrm{w}^{s}\right)=M$ and $\operatorname{nor}(s(\ell))>0$ for all $\ell \geq M$ in $\mathrm{w}^{s}$, then $r \wedge \eta$ essentially decides $\underset{\sim}{\tau}$.

Proof of (5.3.2) $\eta$ extends some $\eta^{k+1} \in \operatorname{poss}(p,<M)$; so $s \leq r \wedge \eta \leq p^{k+1} \leq$ $\tilde{p}^{k+1}$. All we have to show is that $p^{k+1}$ was constructed using the "decide" case. Assume towards a contradiction that the "halve" case was used. Then $s$ is stronger than half $\left(\tilde{p}^{k+1}, \geq M\right)$, so we can unhalve it (using Lemma 5.2.3) to get some $s^{\prime} \leq \tilde{p}^{k+1}$ with large norm such that $s^{\prime} \leq^{*} s$, showing that we could have used the "decide" case after all. This ends the proof of (5.3.2).

\section{Iterations of the mini-steps; the condition $q$.}

Given $p_{0}, M_{0}, n_{0}$ as in the statement of the Lemma, we inductively construct conditions $p_{k}$ and natural numbers $M_{k}$ for $k \geq 1$. Given $p_{k}$ and $M_{k}$, our construction of $p_{k+1}$ and $M_{k+1}$ is as follows: Choose $M_{k+1} \in \mathrm{w}^{p_{k}}$ bigger than $M_{k}$ such that

$$
\operatorname{nor}\left(p_{k}(h)\right)>k+n_{0}+3 \text { for all } h \in \mathrm{w}^{p_{k}} \backslash M_{k+1} \text {. }
$$

Then set $p_{k+1}^{\prime}=r\left(p_{k}, M_{k+1}, k+n_{0}+3\right)$, and construct $p_{k+1}$ by gluing together everything between (including) $M_{k}$ and (excluding) $M_{k+1}$.

The sequence of conditions $\left(p_{k}\right)_{k \in \omega}$ converges to a condition of $\mathbb{Q}$, which we will denote by $q$. Note that $r \leq q$ implies that $\mathrm{w}^{r}$ is a subset of $\left(\mathrm{w}^{p_{0}} \cap M_{0}\right) \cup$ $\left\{M_{0}, M_{1}, M_{2}, \ldots\right\}$ (as we have glued everything between each $M_{i}$ and $M_{i+1}$ ).

It is clear that $q \leq p_{0}$, and that nor $(q, h)>n_{0}+1$ for all $h \in \mathrm{w}^{q} \backslash M_{0}$.

We will later show that $q$ essentially decides $\underset{\sim}{\tau}$ (thus proving the lemma).

The following property will be central:

Assume that $\eta \in \operatorname{poss}\left(q,<M_{\ell}\right)$ for some $\ell \in \omega$, and $r \leq q \wedge \eta$ essentially decides $\underset{\sim}{\tau}$ and $\min \left(\mathrm{w}^{r}\right)=M_{\ell}$ and each $r(m)$ has norm $>1$ for each $m \in \mathrm{w}^{r}$.

Then $q \wedge \eta$ essentially decides $\underset{\sim}{\tau}$. 
Proof of (5.3.3): $\eta$ (or rather: a restriction of $\eta$ to $\operatorname{supp}(p)$ ) was considered as a possible trunk $\eta^{k+1}$ in the "mini-step" when constructing $r\left(p_{\ell-1}, M_{\ell}, \ell+n_{0}+2\right)$. So we can use (5.3.2). This ends the proof of (5.3.3).

3. Using bigness to thin out $q$ to prove essentially deciding.

We now repeat the construction of the proof of Lemma 5.1.5, but this time we do not homogenize on the potential values of some $\underset{\sim}{x}$, but rather on whether $q \curlywedge \eta$ essentially decides $\underset{\sim}{\tau}$ or not.

For now, fix a sublevel $\mathbf{u}=(\ell,-1)$ above $\left(M_{0},-1\right)$ with $\ell \in \mathrm{w}^{q}$.

- We set $\mathfrak{d}_{\mathbf{u}}^{\mathbf{u}}$ to be the collection of Sacks columns $q(\mathbf{u})$. We set $B_{\mathbf{u}}^{\mathbf{u}}$ to be the set of $\eta \in \operatorname{poss}(q,<\mathbf{u})$ such that $q \curlywedge \eta$ essentially decides $\tau$.

- By downwards induction on $\mathbf{u}^{\prime} \in \operatorname{sblvls}(q),\left(M_{0},-1\right) \leq \mathbf{u}^{\prime}<\mathbf{u}$, we construct $\mathfrak{d}_{\mathbf{u}^{\prime}}^{\mathbf{u}}$ and $B_{\mathbf{u}^{\prime}}^{\mathbf{u}}$ such that the following is satisfied:

$-\mathfrak{d}_{\mathbf{u}^{\prime}}^{\mathbf{u}}$ is a strengthening of the subatom (or: collection of Sacks columns) $q\left(\mathbf{u}^{\prime}\right)$, the norm decreases by at most 1 .

- (Homogeneity) $B_{\mathbf{u}^{\prime}}^{\mathbf{u}}$ is a subset of $\operatorname{poss}\left(q,<\mathbf{u}^{\prime}\right)$, such that for each $\eta \in B_{\mathbf{u}^{\prime}}^{\mathbf{u}}$ and each $v \in \operatorname{poss}\left(\mathfrak{d}_{\mathbf{u}^{\prime}}^{\mathbf{u}}\right) \eta \frown v \in B_{\mathbf{u}^{\prime}+1}^{\mathbf{u}}$; and analogously for each $\eta \in$ $\operatorname{poss}\left(q,<\mathbf{u}^{\prime}\right) \backslash B_{\mathbf{u}^{\prime}}^{\mathbf{u}}$ and each $v \in \operatorname{poss}\left(\mathfrak{d}_{\mathbf{u}^{\prime}}^{\mathbf{u}}\right), \eta \frown v \notin B_{\mathbf{u}^{\prime}+1}^{\mathbf{u}}$.

(Just as in the case of rapid reading, we can find these objects using bigness: Assume that $\mathbf{u}^{\prime \prime}$ is the $\operatorname{sblvls}(q)$-successor of $\mathbf{u}^{\prime}$; by induction there is a function $F$ which maps each $\eta \in \operatorname{poss}\left(q,<\mathbf{u}^{\prime \prime}\right.$ to $\{\in B, \notin B\}$; we thin out $q\left(\mathbf{u}^{\prime}\right)$ to $\mathfrak{d}_{\mathbf{u}^{\prime}}^{\mathbf{u}}$ such that for each $v \in \operatorname{poss}\left(q,<\mathbf{u}^{\prime}\right)$ each extension of $v$ compatible with $\mathfrak{d}_{\mathbf{u}^{\prime}}^{\mathbf{u}}$ has the same $F$-value $F^{*}(v)$; this in turn defines $B_{\mathbf{u}^{\prime}}^{\mathbf{u}}$.)

- Assume that $\mathbf{v}<\mathbf{u}$ as above, that $\eta \in \operatorname{poss}(q,<\mathbf{v})$, that $q \curlywedge \eta$ essentially decides $\underset{\sim}{\tau}$ and that $\eta^{\prime} \in \operatorname{poss}(q,<\mathbf{u})$ extends $\eta$. Then trivially $q \curlywedge \eta^{\prime}$ also essentially decides $\underset{\sim}{\tau}$. So we get:

If $q \curlywedge \eta$ essentially decides $\underset{\sim}{\tau}$ for $\eta \in \operatorname{poss}(q,<\mathbf{v})$, then $\eta \in B_{\mathbf{v}}^{\mathbf{u}}$ for any $\mathbf{u}>\mathbf{v}$.

- We now show the converse:

Whenever $\eta \in B_{\mathbf{u}^{\prime}}^{\mathbf{u}}$ for some sublevel $\mathbf{u}^{\prime}$ of the form $\left(M_{\ell^{\prime}},-1\right) \leq \mathbf{u}$ for some $\ell^{\prime}$, then $q \wedge \eta$ essentially decides $\underset{\sim}{\tau}$.

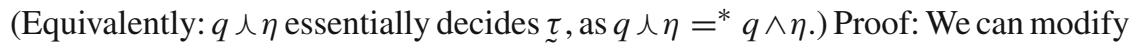
$q$ to a stronger condition $r$ using $\eta$ as trunk and using $\mathfrak{d}_{\mathbf{u}^{\prime \prime}}^{\mathbf{u}}$ for all $\mathbf{u}^{\prime} \leq \mathbf{u}^{\prime \prime} \leq \mathbf{u}$. Any $\eta^{\prime} \in \operatorname{poss}(r,<\mathbf{u})$ is in $B_{\mathbf{u}}^{\mathbf{u}}$, so $q \curlywedge \eta^{\prime}={ }^{*} r \curlywedge \eta^{\prime}$ essentially decides $\underset{\sim}{\tau}$. So $r$ essentially decides $\underset{\sim}{\tau}$. Also, each compound creature in $r$ has norm $>1$, so we can use (5.3.3). This ends the proof of (5.3.5).

- So to show that $q$ essentially decides $\underset{\sim}{\tau}$, it is enough to show that for all $\eta \in$ $\operatorname{poss}\left(q,<\left(M_{0},-1\right)\right)$ there is a $\mathbf{u}$ such that $\eta \in B_{\left(M_{0},-1\right)}^{\mathbf{u}}$.

- As in the rapid reading case, we choose an "infinite branch" $\left(\mathfrak{d}_{\mathbf{v}}^{*}, B_{\mathbf{v}}^{*}\right)$. I.e.: for each $\mathbf{v}^{\prime}$ there is a $\mathbf{u}>\mathbf{v}^{\prime}$ such that $\left(\mathfrak{d}_{\mathbf{v}}^{\mathbf{u}}, B_{\mathbf{v}}^{\mathbf{u}}\right)=\left(\mathfrak{d}_{\mathbf{v}}^{*}, B_{\mathbf{v}}^{*}\right)$ for each $\mathbf{v} \leq \mathbf{v}^{\prime}$. This defines a condition $q_{1} \leq q$.

- To show that $q$ essentially decides $\underset{\sim}{\tau}$, it is enough to show $\eta \in B_{\left(M_{0},-1\right)}^{*}$ for all $\eta \in \operatorname{poss}\left(q,<M_{0}\right)=\operatorname{poss}\left(q_{1},<\left(M_{0},-1\right)\right)$. 
So fix such an $\eta$. Find any $r \leq q_{1} \wedge \eta$ deciding $\underset{\sim}{\tau}$. Without loss of generality, $\min \left(\mathrm{w}^{r}\right)=M_{\ell}$ for some $\ell$, and each compound creature in $r$ has norm at least 1 . Let $\eta^{\prime}>\eta$ be the trunk of $r$ (restricted to $\operatorname{supp}(q)$ and $M_{\ell}$ ). According to (5.3.3), $q \wedge \eta^{\prime}$ essentially decides $\underset{\sim}{\tau}$.

Pick some $\mathbf{u}>\left(M_{\ell},-1\right)$ such that $\left(\mathfrak{d}_{\mathbf{v}}^{\mathbf{u}}, B_{\mathbf{v}}^{\mathbf{u}}\right)=\left(\mathfrak{d}_{\mathbf{v}}^{*}, B_{\mathbf{v}}^{*}\right)$ for each $\mathbf{v} \leq\left(M_{\ell},-1\right)$. According to (5.3.4), $\eta^{\prime} \in B_{\mathbf{v}}^{*}$. By homogeneity, $\eta \in B_{\left(M_{0},-1\right)}^{*}$. So according to (5.3.5), $q \wedge \eta$ essentially decides $\underset{\sim}{\tau}$.

\subsection{Properness, $\omega^{\omega}$-bounding, rapid reading, no randoms}

A standard argument now gives the following:

Theorem 5.4.1 $\mathbb{Q}$ satisfies (the finite/ $\omega^{\omega}$-bounding version of) Baumgartner's Axiom A, in particular it is proper and $\omega^{\omega}$-bounding and (assuming $\mathrm{CH}$ in the ground model) preserves all cofinalities. Also, $\mathbb{Q}$ rapidly reads every $\underset{\sim}{r} \in 2^{\omega}$.

Proof We already know that we can rapidly read each real if we can continuously read it.

We define $q \leq_{n} p$ as: $q \leq p$ and there is an $h \in \mathrm{w}^{q}, h \geq n$, such that $q$ and $p$ are identical below $h$ and $\operatorname{nor}(q(\ell))>n$ for all $\ell \geq h$.

It is clear that any sequence $p_{0} \geq_{0} p_{1} \geq_{1} p_{2} \geq_{2} \ldots$ has a limit; and Lemma 5.3.1 shows that for any name $\underset{\sim}{\tau}$ of an ordinal, $n \in \omega$ and $p \in \mathbb{Q}$, there is a $q \leq_{n} p$ such that modulo $q$ there are only finitely many possibilities for $\underset{\sim}{\tau}$.

Rapid reading gives us:

Lemma 5.4.2 Every new real is contained in a ground model null set, i.e., no random reals are added. So assuming $\mathrm{CH}$ in the ground model, we will have $\operatorname{cov}(\mathcal{N})=\aleph_{1}$ in the extension.

Proof Let $\underset{\sim}{r}$ be the name of an element of $2^{\omega}$ and $p$ a condition. Let $q \leq p$ rapidly read $\underset{\sim}{r}$. So for all $\ell \in \mathrm{w}^{q}, \underset{\sim}{r} \uparrow H(<\ell)$ is determined by each $\eta \in \operatorname{poss}(q,<\ell)$. Hence, the set $\tilde{A}_{\ell}^{q}$ of possibilities for $\underset{\sim}{r} \uparrow H(<\ell)$ has size at most $\operatorname{maxposs}(<\ell)<H(<\ell)<2^{H(<\ell)} / \ell$. So $A_{\ell}^{q}$ has "relative size" $<1 / \ell$, and the sequence $\left(A_{\ell}^{q}\right)_{\ell \in \omega}$ defines (in the ground model) the null set

$$
N=\left\{s \in 2^{\omega}:\left(\forall \ell \in \mathrm{w}^{q}\right) s \uparrow H(<\ell) \in A_{\ell}^{q}\right\} .
$$

And $q$ forces that $\underset{\sim}{r} \in N$.

\section{The specific forcing and the main theorem}

\subsection{The forcing}

Recall that $\Xi$ is partitioned into $\Xi_{\mathrm{sk}}, \Xi_{1 \mathrm{i}}$ and $\Xi_{1 \mathrm{~s}}$. We now further partition $\Xi_{1_{\mathrm{s}}}$ into $\Xi_{\mathrm{nn}}$ and $\Xi_{\mathrm{Cn}}$. So every $\xi \in \Xi$ has one of the following four types: 
- type sk (Sacks) for $\xi \in \Xi_{\mathrm{Sk}}$,

- type cn (cofinality null) for $\xi \in \Xi_{\mathrm{cn}}$,

- type nn (non null) for $\xi \in \Xi_{\mathrm{nn}}$, and

- type $\mathrm{nm}$ (non meager) for $\xi \in \Xi_{1 i}$. So $\mathrm{nm}$ is the only lim-inf type.

Let $\kappa_{t}$ be the size of $\Xi_{t}$.

In the inductive construction of $\mathbb{Q}$ in Sect. 4 , several assumptions are made in the subatom stages $\mathbf{u}$. We will satisfy those assumptions in the following way:

For each type $t \in\{\mathrm{cn}, \mathrm{nn}, \mathrm{nm}\}$ we assume that we have a family of subatomic families $\mathrm{K}_{t, b}^{\prime}$ indexed by a parameter $b$, such that for each $b \in \omega, \mathrm{K}_{t, b}^{\prime}$ is a subatomic family living on some $\operatorname{POSS}_{t, b}^{\prime}$ satisfying $b$-bigness. Actually, we will require a stronger variant of $b$-bigness such that we can find an homogeneous successor subatom while decreasing the norm not by 1 but by at most $1 / b$. I.e., we require:

For $x \in \mathrm{K}_{t, b}^{\prime}$ and $F: \operatorname{poss}(x) \rightarrow b$ there is a $y \leq x$ such that $\operatorname{nor}(y) \geq \operatorname{nor}(x)-1 / b$ and $F\lceil\operatorname{poss}(y)$ is constant.

Additionally we require that

there is at least one subatom in $\mathrm{K}_{t, b}^{\prime}$ with norm $\geq b$.

Then we set for each subatomic sublevel $\mathbf{u}=(\ell, j)$

$$
b(\mathbf{u}):=B(\mathbf{u}) \cdot(b(\mathbf{v})+1)+1,
$$

where $\mathbf{v}$ is the largest ${ }^{31}$ subatomic sublevel smaller than $\mathbf{u}$. So the sequence $b(\mathbf{u})$ is strictly (actually: very quickly) increasing. According to the definition 4.0.2 of $B(\mathbf{u})$, we also get:

Lemma 6.1.4 $b(\mathbf{u}) \geq 2 \cdot \operatorname{maxposs}(<\mathbf{u})$, and even $b(\mathbf{u}) \geq 2^{\text {(number of sublevels below } \mathbf{u}) \cdot \operatorname{maxposs}(<\mathbf{u})}$.

Then we set (for all $\xi \in \Xi_{t}$ )

$$
\mathrm{K}_{\xi, \mathbf{u}}:=\mathrm{K}_{t, b(\mathbf{u})}^{\prime}
$$

This way we automatically satisfy requirements (b) and (c) of item $(* 5)$ on page 1070 . And since there are only four, i.e., finitely many, types, there is automatically a bound $M$ on $\left|\operatorname{POSS}_{\xi, \mathbf{u}}\right|$ as required in (d).

Strong bigness gives us the following property:

Lemma 6.1.5 Let I be a finite set of subatomic sublevels (and thus I is naturally ordered). Let $\mathbf{v}$ be the minimum of $I$. For each $\mathbf{u} \in I$ let $\xi_{\mathbf{u}} \in$ non-sk and $x_{\mathbf{u}} a$ subatom in $K_{\xi_{\mathbf{u}}, \mathbf{u}}$. Let $F: \prod_{\mathbf{u} \in I} \operatorname{poss}\left(x_{\mathbf{u}}\right) \rightarrow b(\mathbf{v})$. Then there are $y_{\mathbf{u}}<x_{\mathbf{u}}$ with $\operatorname{nor}\left(y_{\mathbf{u}}\right) \geq \operatorname{nor}\left(x_{\mathbf{u}}\right)-1 / b(\mathbf{u})$ and such that $F \uparrow \prod_{\mathbf{u} \in I} \operatorname{poss}\left(y_{\mathbf{u}}\right)$ is constant.

31 If $\mathbf{u}$ is $(0,0)$, the smallest of all subatomic sublevels, we just set $b(\mathbf{u}):=B(\mathbf{u})$. By the way, it would be enough to set $b(\mathbf{u}):=B(\mathbf{u})$, as this sequence would be increasing sufficiently fast, but this would require two extra lines of calculations. 
Proof We construct $y_{\mathbf{u}}$ by downwards induction on $\mathbf{u} \in I$ : Let $\mathbf{u}^{\prime}$ be the maximum of $I$, then $F$ can be written as function from $\operatorname{poss}\left(x_{\mathbf{u}^{\prime}}\right)$ to $b(\mathbf{v})^{P}$, where $P=\prod_{\mathbf{u} \in I \backslash\left\{\mathbf{u}^{\prime}\right\}} \operatorname{poss}\left(x_{\mathbf{u}}\right)$. As $|P|$ is less than the number of sublevels below $\mathbf{u}^{\prime}$ times $\operatorname{maxposs}\left(<\mathbf{u}^{\prime}\right)$, we get $|P|<b\left(\mathbf{u}^{\prime}\right)$, and thus can use strong bigness to get $y_{\mathbf{u}^{\prime}}<x_{\mathbf{u}^{\prime}}$.

Now continue by induction.

The families $\mathrm{K}_{t, r}^{\prime}$ that we will actually use are described in Sect. 10 for $t=\mathrm{cn}$, Sect. 8 for $t=\mathrm{nn}$, and Sect. 7 for $t=\mathrm{nm}$.

In addition, we will define there for each $\mathrm{K}_{t, b}^{\prime}$ a number $H^{\prime}(t,=b)$, and in the inductive construction, we define $H$ as follows:

Definition $H(<(0,-1)):=3$. If $\mathbf{u}=(\ell, j)$ is a sublevel with immediate predecessor $\mathbf{u}^{\prime}$, we define $H(<\mathbf{u})=H\left(\leq \mathbf{u}^{\prime}\right)$ in cases by:

- For a Sacks sublevel u (i.e., $j=-1), H(<\ell)=H(<\mathbf{u}):=2+\ell+\operatorname{maxposs}(<\ell)+$ $H\left(<\mathbf{u}^{\prime}\right)+\max \left(\left\{H^{\prime}\left(t,=b\left(\mathbf{u}^{\prime}\right)\right): t \in\{\mathrm{nm}, \mathrm{nn}, \mathrm{cn}\}\right\}\right)$.

- For $j=0: H(<\mathbf{u}):=1+H\left(<\mathbf{u}^{\prime}\right)+\max \left(I_{\mathrm{sk}, \ell}\right)$.

- For $\left.j>0, H(<\mathbf{u}):=1+H\left(<\mathbf{u}^{\prime}\right)+\max \left\{H^{\prime}\left(t,=b\left(\mathbf{u}^{\prime}\right)\right): t \in\{\mathrm{nm}, \mathrm{nn}, \mathrm{cn}\}\right\}\right)$.

So in particular, if $p$ rapidly reads $\underset{\sim}{r}$, then for all $t \in\{\mathrm{nm}, \mathrm{nn}, \mathrm{cn}\}$ and all subatomic sublevels $\mathbf{u}$

$$
\underset{\sim}{r} H^{\prime}(t,=\mathbf{u}) \text { is decided } \leq b(\mathbf{u}) .
$$

Note that once we fix the parametrized subatomic families $\mathrm{K}_{t, b}^{\prime}$ and $H^{\prime}(t,=b$ ) (and the cardinalities $\kappa_{t}$ ), we have specified everything required to construct $\mathbb{Q}$, and $\mathbb{Q}$ will satisfy Baumgartner's Axiom A, will be $\omega^{\omega}$-bounding, and, assuming $\mathrm{CH}$, will have the $\aleph_{2}$-cc. We also get rapid reading.

\subsection{The main theorem}

We will show:

Theorem 6.2.1 Assume (in V) CH, $\kappa_{\mathrm{nm}} \leq \kappa_{\mathrm{nn}} \leq \kappa_{\mathrm{Cn}} \leq \kappa_{\mathrm{sk}}$ and $\kappa_{t}^{\aleph_{0}}=\kappa_{t}$ for $t \in\{\mathrm{nm}, \mathrm{nn}, \mathrm{cn}, \mathrm{sk}\}$. Then there is a forcing $\mathbb{Q}$ which forces

(1) $\operatorname{cov}(\mathcal{N})=\mathfrak{d}=\aleph_{1}$,

(2) $\operatorname{non}(\mathcal{M})=\operatorname{cof}(\mathcal{M})=\kappa_{\mathrm{nm}}$,

(3) $\operatorname{non}(\mathcal{N})=\kappa_{\mathrm{nn}}$,

(4) $\operatorname{cof}(\mathcal{N})=\kappa_{\mathrm{Cn}}$,

(5) $2^{\aleph_{0}}=\kappa_{\mathrm{sk}}$.

Moreover, $\mathbb{Q}$ preserves all cardinals and all cofinalities.

As mentioned above, we fix disjoint index sets $\Xi_{t}(t \in\{\mathrm{sk}, \mathrm{cn}, \mathrm{nn}, \mathrm{nm}\})$ of respective sizes $\kappa_{t}$, and we construct $\mathbb{Q}$ as described above. Then the following points are obvious or have already been shown:

(1) $\mathfrak{d}=\aleph_{1}$, since $\mathbb{Q}$ is $\omega^{\omega}$-bounding. And it was already shown in Lemma 5.4.2 that no random reals are added, $\operatorname{so} \operatorname{cov}(\mathcal{N})=\aleph_{1}$. 
(5) If $\alpha \neq \beta \in \Xi_{\mathrm{Sk}}$, then the generic reals at $\alpha$ and $\beta$ are forced to be different, so we have at least $\kappa_{\mathrm{sk}}$ many reals. Every real in the extension is read continuously, so by Lemma 5.1.4 there are at most $\kappa_{\mathrm{sk}}^{\aleph_{0}}=\kappa_{\mathrm{sk}}$ many reals.

(•) The "moreover" part is clear because $\mathbb{Q}$ satisfies Baumgartner's Axiom A and has the $\aleph_{2}$-cc.

In the rest of the paper, we will describe the families $K_{t, b}^{\prime}$ and $H^{\prime}(t,=b)$ and prove the remaining parts of the main theorem:

(2) $\operatorname{In} \mathrm{ZFC}, \max (\mathfrak{d}, \operatorname{non}(\mathcal{M}))=\operatorname{cof}(\mathcal{M})$. And $\operatorname{non}(\mathcal{M}) \leq \kappa_{\mathrm{nm}}$ is shown in 6.4.1, and $\geq$ in 7.3.2.

(3) $\operatorname{non}(\mathcal{N}) \leq \kappa_{\mathrm{nn}}$ is shown in 10.5.2; and $\geq$ in 8.3.2.

(4) $\operatorname{cof}(\mathcal{N}) \leq \kappa_{\mathrm{Cn}}$ is shown in 6.3.4; and $\geq$ in 10.4.2.

\subsection{The Sacks part: $\operatorname{cof}(\mathcal{N}) \leq \kappa_{\mathrm{cn}}$}

We will show that every null set added by $\mathbb{Q}$ is contained in a null set which is already added by the non-Sacks part.

We will first show that the quotient $\mathbb{Q} / \mathbb{Q} \Xi_{\text {non-sk }}$ (in other words: the extension from the universe obtained not using the Sacks coordinates to the full generic extension) has the Sacks property.

Recall that the Sacks property states (or, depending on the definition, is equivalent to): Every function in $\omega^{\omega}$ in the extension is caught by an $(n+2)$-slalom from the ground model. (I.e., there is a function $S: \omega \rightarrow[\omega]^{<\omega}$ in the ground model with $|S(n)| \leq n+2$, and $f(n) \in S(n)$ for all $n$.)

The Laver property is similar, but only applies to functions $f$ in the extension which are bounded by a ground model function.

We get

\section{Lemma 6.3.1 (1) Laver property is equivalent to:}

Whenever $\underset{\sim}{\underset{\sim}{r}} \in 2^{\omega}$ is in the extension and $G: \omega \rightarrow \omega$ in the ground model, then there is in the ground model a tree $T$ (without terminal nodes) such that $\underset{\sim}{r} \in[T]$ and $\left|T \uparrow 2^{G(n)}\right|<n+2$ for all $n$.

(2) The Sacks property is equivalent to the conjunction of Laver property and $\omega^{\omega}$ bounding.

(3) If an extension has the Sacks property, then any new null set is contained in an old null set.

Proof For the well known (2) and (3) see, e.g., [3, Theorem 2.3.12]. For (1), we only show how to get the Laver property (which is enough for this paper, and the other direction is similarly easy).

Suppose that $g: \omega \rightarrow \omega$ is given. Enumerate $\{(n, m): m \leq g(n)\}$ in lexicographic order as $\left(n_{i}, m_{i}\right)$. Define a function $G: \omega \rightarrow \omega$ by

$$
G(n)=\min \left\{i: n_{i}>n\right\}=n+1+\sum_{k \leq n} g(k) .
$$


(For convenience we will think of $G(-1)=0$.) Note that according to the enumeration given above, every function $r: \omega \rightarrow 2$ determines a subset of $\prod_{n<\omega}(g(n)+1)$ by $\left\{\left(n_{i}, m_{i}\right): r(i)=1\right\}$. Accordingly, certain functions $r$ induce a function bounded by $g$ : those functions $r$ such that given any $n$ there is a unique $m \leq g(n)$ such that $(n, m)$ is in the subset determined by $r$ as described above. (Equivalently, for each $n$ there is a unique $G(n-1) \leq i<G(n)$ such that $r(i)=1$.) Given such an $r$, by $\operatorname{val}(r, n)$ we denote $m_{i}$ where $G(n-1) \leq i<G(n)$ is such that $r(i)=1$.

Note that given any function $f$ bounded by $g$ there is a unique function $r_{f}: \omega \rightarrow 2$ (which determines a function bounded by $g$ as described above) such that val $\left(r_{f}, n\right)=$ $f(n)$ for all $n$.

Suppose that $f$ is a name for a function bounded by the ground model function $g$. Let $\underset{\sim}{r}$ be a name for the function $\omega \rightarrow 2$ as described above, and let $T$ be the tree guaranteed to exist by the assumption (using the function $G$ defined from $g$ above). We may assume that all branches $x$ of $T$ determine a function bounded by $g$ as described above. Now define a slalom $S$ by $S(n)=\{\operatorname{val}(x, n): x \in[T]\}$. It is clear that $S$ catches $\underset{\sim}{f}$.

We now prove our version of the Laver property for the quotient. As the whole forcing is $\omega^{\omega}$-bounding, this implies the Sacks property.

Lemma 6.3.2 (1) Assume that $p$ is a condition, $\underset{\sim}{r} \in 2^{\omega}$ a name and $G: \omega \rightarrow \omega$ is in $V$. Then there is a $q \leq p$ and a name $\underset{\sim}{T} \subseteq 2^{<\omega}$ (of a tree without terminal nodes) such that: $q$ continuously reads $\underset{\sim}{T}$ not using any Sacks indices; $q$ forces $r \in[\underset{\sim}{T}] ;$ and $|\underset{\sim}{T}| 2^{G(n)} \mid<n+2$ for all $n$.

(2) Therefore the quotient $\mathbb{Q} / \mathbb{Q} \Xi_{\text {non-sk }}$ has the Laver property (and thus the Sacks property).

Proof If $G_{1}(n) \leq G_{2}(n)$ for all $n$, and $\underset{\sim}{T}$ witnesses the conclusion of the lemma for $G_{2}$, then $\underset{\sim}{T}$ also witnesses the lemma for $G_{1}$. So we may without loss of generality increase the function $G$ whenever this is convenient.

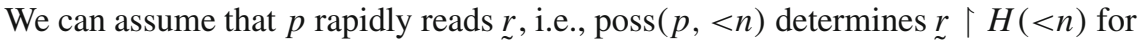
all $n \in \mathrm{w}^{p}$.

We can then assume that there is a strictly increasing function $G^{\prime}$ such that $G^{\prime}(n) \in$ ${ }_{\mathrm{w}}{ }^{p}$ and $G(n)=H\left(<G^{\prime}(n)\right)$ for all $n$ (as we can increase $G$ ).

Also, to simplify notation, we can assume that $\mathrm{w}^{p}=\left\{G^{\prime}(0), G^{\prime}(1), \ldots\right\}$. (Otherwise, just glue.)

So each $\eta \in \operatorname{poss}\left(p,<G^{\prime}(n)\right)$ determines a value for $\underset{\sim}{r} \uparrow G(n)$, which we call $R^{n}(\eta)$. We view $\eta$ as a pair $\left(\eta_{\mathrm{sk}}, \eta_{\text {non-sk }}\right)$ for $\eta_{t}:=\eta \uparrow \Xi_{t}$ for $t \in$ \{non-sk, sk\}. Accordingly we write $R^{n}\left(\eta_{\mathrm{sk}}, \eta_{\text {non-sk }}\right)$. If we fix $\eta_{\mathrm{sk}}$, then $R^{n}\left(\eta_{\mathrm{sk}},-\right)$ can be viewed as a name (for an element of $2^{G(n)}$ ) which does not depend on the Sacks part, in the following way: If there is a $\eta_{\text {non-sk }}$ compatible with the generic filter such that $\left(\eta_{\mathrm{sk}}, \eta_{\text {non-sk }}\right)=\eta \in \operatorname{poss}\left(p,<G^{\prime}(n)\right)$, then the value is $R^{n}(\eta)$ (and otherwise $\emptyset$, say).

Below we will construct $q \leq p$ by gluing and by strengthening Sacks columns (and we will leave the support, the subatoms and the halving parameters unchanged).

Assume we have such a $q$, and assume that $G^{\prime}\left(m_{0}\right)<G^{\prime}\left(m_{1}\right)$ are consecutive elements of $\mathrm{w}^{q}$. Note that $G^{\prime}\left(m_{0}\right)<G^{\prime}\left(m_{0}+1\right)<\cdots<G^{\prime}\left(m_{1}-1\right)<G^{\prime}\left(m_{1}\right)$ are 
consecutive elements of $\mathrm{w}^{p}$. Fix $\eta \in \operatorname{poss}\left(q,<G^{\prime}\left(m_{1}\right)\right)$ and $m_{0} \leq \ell \leq m_{1}$. Then $\eta$ extends a unique element of $\operatorname{poss}\left(q,<G^{\prime}(\ell)\right)$, which we call $\eta^{\ell}$. We can then restrict $\eta^{\ell}$ to the Sacks part: $\eta_{\mathrm{sk}}^{\ell}:=\eta^{\ell} \uparrow \Xi_{\mathrm{sk}}$.

Note:

- $\eta_{\mathrm{sk}}^{\ell}$ is $\eta$ restricted to the Sacks part and to "height $G^{\prime}(\ell)$ ", i.e.,

$$
\eta_{\mathrm{sk}}^{\ell}:=\eta \uparrow \Xi_{\mathrm{sk}} \times\left(1+\max \left(I_{\mathrm{sk}, G^{\prime}(\ell)}\right)\right)
$$

- $q \wedge \eta$ forces that the name $R^{\ell}\left(\eta_{\mathrm{sk}}^{\ell},-\right)$ (which does not depend on the Sacks part) is evaluated to $\underset{\sim}{r}\lceil G(\ell)$.

- So $q$ forces that $\underset{\sim}{r} \uparrow G(\ell)$ is an element of

$$
{\underset{\sim}{T}}^{\ell}:=\left\{R^{\ell}\left(\eta_{\mathrm{sk}}^{\ell},-\right): \eta \in \operatorname{poss}\left(q,<G^{\prime}\left(m_{1}\right)\right)\right\},
$$

a name not depending on the Sacks part.

So it is enough to show that there are few $\eta_{\mathrm{sk}}^{\ell}$, i.e.,

$$
\left|S_{\ell}\right|<\ell+2 \text { for } S_{\ell}:=\left\{\eta_{\mathrm{sk}}^{\ell}: \eta \in \operatorname{poss}\left(q,<G^{\prime}\left(m_{1}\right)\right)\right\} .
$$

We will now by induction on $n$ :

(1) construct $h_{n}$, where $\mathrm{w}^{q}$ will be the set $\left\{G^{\prime}\left(h_{0}\right), G^{\prime}\left(h_{1}\right), \ldots\right\}$;

(2) construct $q$ below $G^{\prime}\left(h_{n}\right)$,

(3) and show that $\left(\star_{\ell}\right)$ holds for all $\ell \leq h_{n}$.

We set $h_{0}=0$; so $G^{\prime}\left(h_{0}\right)=\min \left(\mathrm{w}^{p}\right)$ and $q$ below $G^{\prime}\left(h_{0}\right)$ has to be identical to $p$. And $\left(\star_{0}\right)$ holds, as $S_{0}$ is a singleton.

Assume we have already constructed $h_{n}$ and $q$ below $G^{\prime}\left(h_{n}\right)$, satisfying $\left(\star_{\ell}\right)$ for $\ell \leq h_{n}$.

(1) For any $I$ and $\mathfrak{s} \subseteq 2^{I}$, we write nor Sacks $^{*}(\mathfrak{s})$ for $\operatorname{nor}_{\text {Sacks }}^{B\left(G^{\prime}\left(h_{n}\right)\right), G^{\prime}\left(h_{n}\right)}(\mathfrak{s})$, see 2.3.5. (I.e., the Sacks norm that would be assigned to a Sacks column starting at $G^{\prime}\left(h_{n}\right)$

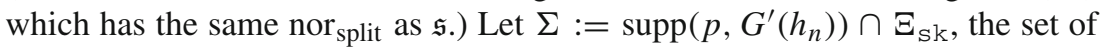
Sacks indices active at the current level. Let $s$ be minimal such that nor ${ }_{\text {Sacks }}^{*}\left(2^{s}\right) \geq$ $n$, and define $h^{\prime}$ by

$$
h^{\prime}:=\left(h_{n}+1\right) \cdot 2^{s \cdot|\Sigma|} \text {. }
$$

Finally, let $h_{n+1}$ be minimal such that for all $\xi \in \Sigma$ there is an $\ell(\xi)$ with $h^{\prime} \leq \ell(\xi)<h_{n+1}$ and $\operatorname{nor}_{\text {Sacks }}^{*}\left(p\left(\xi, G^{\prime}(\ell(\xi))\right)\right) \geq n$. (We can find such $\ell(\xi)$, as even nor Sacks $\left(p\left(\xi, G^{\prime}(\ell)\right)\right)$ diverges to infinity.)

(2) $G^{\prime}\left(h_{n}\right)<G^{\prime}\left(h_{n}+1\right)<\cdots<G^{\prime}\left(h_{n+1}-1\right)<G^{\prime}\left(h_{n+1}\right)$ are consecutive elements of ${ }^{p}$. We glue $p$ between $G^{\prime}\left(h_{n}\right)$ and $G^{\prime}\left(h_{n+1}-1\right)$, so $G^{\prime}\left(h_{n}\right)$ and $G^{\prime}\left(h_{n+1}\right)$ will be consecutive elements of $\mathrm{w}^{q}$.

We now define the compound creature $q\left(G^{\prime}\left(h_{n}\right)\right)$, a pure strengthening of the compound creature glue $\left(p\left(G^{\prime}\left(h_{n}\right)\right), \ldots, p\left(G^{\prime}\left(h_{n+1}-1\right)\right)\right)$ : The subatoms are unchanged. So we just have to specify for each $\xi \in \operatorname{supp}\left(p, h_{n}\right) \cap \Xi_{\mathrm{sk}}$ the new Sacks column $q\left(\xi, h_{n}\right) \leq p\left(\xi, G^{\prime}\left(h_{n}\right)\right) \otimes \cdots \otimes p\left(\xi, G^{\prime}\left(h_{n+1}-1\right)\right)$ 
as follows: Recall that there is one $\ell(\xi)$ such that $h^{\prime} \leq \ell(\xi)<h_{n+1}$ and $\operatorname{nor}_{\text {Sacks }}^{*}\left(p\left(\xi, G^{\prime}(\ell(\xi))\right)\right) \geq n$. Choose a singleton subset of $p\left(\xi, G^{\prime}(m)\right)$ for all $m \neq \ell(\xi)$, and at $m=\ell(\xi)$ pick a subtree of $p\left(\xi, G^{\prime}(m)\right)$ which is isomorphic to $2^{s}$ (in the sense that each branch has $s$ splitting points).

By the definition of $s$, we have $\operatorname{nor}_{\text {Sacks }}\left(q\left(\xi, h_{n}\right)\right) \geq n$, and therefore $\operatorname{nor}\left(q\left(h_{n}\right)\right) \geq \min \left(n, \operatorname{nor}\left(p\left(h_{n}\right), \ldots, \operatorname{nor}\left(p\left(h_{n+1}-1\right)\right)\right)\right)$. So in particular the $q$ we get after the induction will be an element of $\mathbb{Q}$.

(3) As we choose singletons below $G^{\prime}\left(h^{\prime}\right),\left|S_{h_{n}}\right|=\left|S_{h_{n}+1}\right|=\cdots=\left|S_{h^{\prime}-1}\right|$. By induction, $\left|S_{h_{n}}\right|<h_{n}+2$; so $\left(*_{\ell}\right)$ holds for $\ell \leq h^{\prime}$. For each $h^{\prime} \leq \ell \leq h_{n+1}$, we added at each $\xi \in \Sigma$ at most once at most $2^{s}$ many possibilities. So $\left|S_{\ell}\right| \leq$ $\left(h_{n}+1\right) \cdot 2^{s \cdot|\Sigma|}<\ell+2$, by $(6.3 .3)$.

By Lemma 6.3.1(3), we conclude:

Corollary 6.3.4 (1) If $\underset{\sim}{N}$ is the name of a null set and $p$ a condition, then there is a $q \leq p$ and some name of a null set $\underset{\sim}{N^{\prime}}$ not depending on any Sacks indices such that $q$ forces $\underset{\sim}{N} \subseteq \underset{\sim}{N}$.

(2) $\mathbb{Q}$ forces $\operatorname{cof}(\tilde{\mathcal{N}}) \leq \kappa_{\mathrm{cn}}$.

\subsection{Lim-inf and lim-sup: $\operatorname{non}(\mathcal{M}) \leq \kappa_{\mathrm{nm}}$}

The following does not require any knowledge about the particular subatoms used in the forcing construction, the only relevant fact is that the nm indices are the only ones that use a lim-inf construction.

Lemma 6.4.1 $\mathbb{Q}$ forces $\operatorname{non}(\mathcal{M}) \leq \kappa_{\mathrm{nm}}$.

Proof We claim that the set of all reals that can be read continuously from nm-indices is not meager. This set has size $\leq \kappa_{\mathrm{nm}}$ by Lemma 5.1.4.

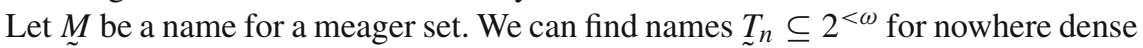
trees such that $\underset{\sim}{M}=\bigcup_{n \in \omega}[\underset{\sim}{T}]$ is forced. We want to show that we can continuously read a real $\underset{\sim}{r} \notin M$ using only the nm-indices.

As $\mathbb{Q}$ is $\omega^{\omega}$-bounding and $T_{n}$ is nowhere dense, there is in $V$ a function $f_{n}: \omega \rightarrow \omega$ such that for each $v \in 2^{k}$ there is a $v^{\prime} \in 2^{f_{n}(k)}$ extending $v$ and not in $\underset{\sim}{T_{n}}$.

We fix some $p \in \mathbb{Q}$ forcing the above, and assume that $p$ is pruned and continuously reads $\underset{\sim}{T_{n}}$ for each $n$. We will construct (in $V$ ) a $q \leq p$ and an $\underset{\sim}{r}$ continuously read by $q$ only using nm indices, such that $q$ forces $\underset{\sim}{r} \notin \underset{\sim}{M}$.

Assume we have already constructed $q$ below some $k_{n} \in \mathrm{w}^{q}$, and that we already have some $h_{n} \in \omega$ and a name $\ell_{n}$ for an element of $2^{h_{n}}$ that is decided by $\operatorname{poss}\left(q,<k_{n}\right) \uparrow$ $\Xi_{\mathrm{nm}}$. (The real $\underset{\sim}{r}$ will be the union of the $\underset{\sim}{\ell_{n}}$.) We also assume that is already guaranteed that ${\underset{\sim}{n}}_{n}$ is not in $\underset{\sim}{T_{0}} \cup \cdots \cup \underset{\sim}{T_{n-1}}$ ).

Enumerate $\operatorname{poss}\left(q,<k_{n}\right)$ as $\eta_{0}, \ldots, \eta_{K-1}$.

Set $k^{0}:=k_{n}, h^{0}:=h_{n},{\underset{\sim}{\ell}}^{0}:={\underset{\sim}{\ell}}_{n}$, and we define $q^{\prime}$ below $k^{0}$ to be $q$. By induction on $r \in K$ we now deal with $\eta_{r}$ : Assume we are given a name $\underset{\sim}{\ell}{ }^{r}$ for an element of $2^{h^{r}}$ that is decided by $\operatorname{poss}\left(q^{\prime},<k^{r}\right) \uparrow \Xi_{\mathrm{nm}}$, and that we have constructed $q^{\prime}$ below $k^{r} \in \mathrm{w}^{p}$, in a way that between $k^{0}$ and $k^{r}$ on the non-nm indices, all subatoms and Sacks columns in $q^{\prime}$ are singletons. 
Set $h^{r+1}:=f_{n}\left(h^{r}\right)$. Choose $k^{r+1} \in \mathrm{w}^{p}$ bigger than $k^{r}$ and large enough to determine $\underset{\sim}{X}:={\underset{\sim}{T}}_{n} \uparrow h^{r+1}$. I.e., there is a function $F$ from $\operatorname{poss}\left(p,<k^{r+1}\right)$ to potential values of $\underset{\sim}{X}$. We now define $q^{\prime}$ between $k^{r}$ and $k^{r+1}$ : The nm-subatoms are unchanged (i.e., the ones of $p$ ), for the other subatoms and Sacks columns, we choose arbitrary singletons. A $v \in \operatorname{poss}\left(p,<k^{r+1}\right)$ consists of: the part below $k^{r}$ called $A$, then non-nm-part above $k^{r}$ called $B$, and the nm-part above $k^{r}$ called $C$. So we can write $\underset{\sim}{X}=F(A, B, C)$. If we assume that the generic chooses $\eta_{r}$ (i.e., $A=\eta_{r}$ ) and then follows the singleton values of $q$ on the non-nm-part (which determines $B$ to be some $B_{q}$ ), then $\underset{\sim}{X}$ can be written as nm-name. More formally: We can define $\underset{\sim}{X}{ }^{\prime}$ as $F\left(\eta_{r}, B_{q},-\right)$, which is a nm-name and forced by $q$ to be $\underset{\sim}{X}$.

Also, we know that $p$ forces that there is an element $\ell^{\prime} \in 2^{h^{r+1}}$ which extends $\underset{\sim}{\ell^{r}}$ (which by induction is already determined by the nm-part of $\eta_{r}$ ) and which is not in $\underset{\sim}{\tilde{X}}$. So (in $V$ ) we can pick for all choices of $C$ an $\ell^{\prime}(C) \in 2^{h^{r+1}} \backslash F\left(\eta_{r}, B_{q}, C\right)$ extending ${\underset{\sim}{\ell}}^{r}$. Then $\underset{\sim}{\ell^{r+1}}=\ell(-)$ is a nm-name determined below $k^{r+1}$, and $q$ forces that $\underset{\sim}{\ell^{n+1}}$

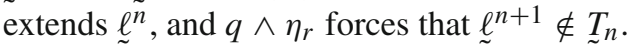

We repeat the construction for all $r \in \tilde{K}$, and set $\ell_{n+1}:=\ell^{K}, h_{n+1}:=h^{K}$ and set $k_{n+1}$ to be the $\mathrm{w}^{p}$-successor of $k^{K}$, where we use the Sacks columns and subatoms of $p$ between $k^{K}$ and $k_{n+1}$. We now glue the condition between $k_{n}$ and $k_{n+1}$. This results in a condition that still has "large" norm, as described in Lemma 3.5.6.

\section{The nm part}

\subsection{The subatomic creatures for type nm}

We now describe the subatomic family $\mathrm{K}_{\mathrm{nm}, b}^{\prime}$ used at nm-indices (depending on the parameter $b$ ).

Definition 7.1.1 (1) Fix a finite index set $I \subseteq \omega$ which is large enough so that item (4) below is satisfied. For notational simplicity, we assume that $I$ is disjoint to all intervals already used. ${ }^{32}$

(2) $\operatorname{POSS}_{\mathrm{nm}, b}:=2^{I}$.

(3) A subatomic creature $x$ is just a nonempty subset of $2^{I}$, where we $\operatorname{set} \operatorname{poss}(x):=$ $x$ and

$$
\operatorname{nor}(x):=\frac{1}{b} \log _{b}(|\operatorname{poss}(x)|)
$$

(4) We require nor(POSS) $>b$ [thus satisfying (6.1.2)].

(5) We set $H^{\prime}(\mathrm{nm}, b):=2^{\max (I)+1}$.

Clearly, the norm satisfies strong $b$-bigness (i.e., satisfies the requirement (6.1.1)).

\footnotetext{
32 This is a bit fuzzy, but it does not matter how we interpret it. More specifically, we could use any of the following: "disjoint to all $I$ that are associated to smaller parameter values $b^{\prime}<b$ ", or: "disjoint to all $I$ that have actually been used in type $\mathrm{nm}$ for some $\mathrm{K}_{\xi, \mathrm{v}}$ "; and since $H^{\prime}(\mathrm{nm},=b)$ is larger than $\max (I)$, it would also follow from: "the minimum of $I$ is bigger than $H\left(<\mathbf{u}^{\prime}\right)$, where $\mathbf{u}^{\prime}$ is the predecessor of the current sublevel".
} 
Note 7.1.2 We just used the simplest possible norm here. It turns out that the details of the definition of this norm are not relevant, as long as the norm has bigness. Later in Sect. 11 we will use a different norm to get a different constellation of cardinal characteristics.

\subsection{The generic object}

Recall that (according to Sect. 6.1) when constructing the forcing at subatomic sublevels $\mathbf{u}$, we use for all $\xi \in \Xi_{\mathrm{nm}}$ the subatomic family $\mathrm{K}_{\xi, \mathbf{u}}=\mathrm{K}_{\mathrm{nm}, b(\mathbf{u})}^{\prime}$ living on some interval $I$, which we will call $I_{\mathrm{nm}, \mathbf{u}}$.

Fix $\alpha$ of type nm. Recall that the generic object $y_{\alpha}$ assigns to each subatomic sublevel $\mathbf{u}$ the element of $\operatorname{POSS}_{\alpha, \mathbf{u}}$ chosen by the generic filter.

We define the name $\underset{\sim}{M_{\alpha}}$ of a meager set as follows:

A real $r \in 2^{\omega}$ is in $M_{\alpha}$ iff for all but finitely many levels $\ell$ there is a subatomic sublevel $\tilde{\mathbf{u}}=(\ell, j)$ such that $r \nmid I_{\mathrm{nm}, \mathbf{u}} \neq y_{\alpha}(\mathbf{u})$.

If $p$ rapidly reads $\underset{\sim}{r}$, then according to (6.1.6) and 7.1.1(5),

$\underset{\sim}{r}\left\lceil I_{\mathrm{nm}, \mathbf{u}}\right.$ is decided $\leq \mathbf{u}$.

Also, since $b(\mathbf{u})>\operatorname{maxposs}(<\mathbf{u})$, we get:

If the norm of a nm-subatom $x$ at sublevel $\mathbf{u}$ is at least 1, then $|\operatorname{poss}(x)|>\operatorname{maxposs}(<\mathbf{u})$.

(Recall Note 7.1.2: This is true whenever the norm has bigness.)

\section{$7.3 \operatorname{non}(\mathcal{M}) \geq \kappa_{\mathrm{nm}}$}

Lemma 7.3.1 Let $\underset{\sim}{r}$ be a name of a real, p a condition that rapidly reads $\underset{\sim}{r}$ not using ${ }^{33}$ $\alpha \in \Xi_{\mathrm{nm}}$. Then $p$ forces that $\underset{\sim}{r} \in \underset{\sim}{M_{\alpha}}$.

Proof It is enough to prove that some $q \leq p$ forces that $\underset{\sim}{\underset{\sim}{\sim}} \in{\underset{\sim}{\alpha}}_{\alpha}$ : Assume that $p$ does not force $\underset{\sim}{r} \in M_{\alpha}$, then some $p^{\prime} \leq p$ forces the negation; $p^{\prime}$ still rapidly reads $\underset{\sim}{r}$ not using $\alpha$, so if we know that there is a $q \leq p^{\prime}$ as claimed, we get a contradiction.

We can assume that $p$ is pruned and that $\alpha \in \operatorname{supp}(p)$. We will construct a $q$ purely stronger than $p$ (in particular with the same $\mathrm{w}$, halving parameters, and trunk). Actually, we will only strengthen one subatom at index $\alpha$ for each level $h \geq \min \left({ }_{\mathrm{w}}{ }^{p}\right)$.

For all $h \geq \min \left(\mathrm{w}^{p}\right)$ (not necessarily in $\mathrm{w}^{p}$ ), there are several $j \in J_{h}$ such that $\operatorname{nor}(x)>1$ for the subatom $x=p(\alpha,(h, j))$. For each such $h$ we pick exactly one subatomic sublevel $\mathbf{u}(h)=(h, j)$, with $x(h)$ the according subatom.

According to (7.2.2), $\underset{\sim}{r} I_{\mathrm{nm}, \mathbf{u}}$ is decided $\leq \mathbf{u}$ and therefore even below $\mathbf{u}$ (since $\alpha$ is the active index at sublevel $\mathbf{u}$; according to modesty no other index can be active; and

33 cf. 5.1.1. 
$\underset{\sim}{r}$ does not depend on $\alpha)$. Therefore there are at most maxposs $(<\mathbf{u})$ many possibilities for $\underset{\sim}{r} \uparrow I_{\mathrm{nm}, \mathbf{u}}$. According to (7.2.3) there has to be at least one element $s$ of $\operatorname{poss}(x(h))$ which differs from all of these possibilities. So we can in $q$ replace the subatom $x(h)$ with the singleton $\{s\}$. Then the norms in $q$ will still be large. (If $A \subseteq J_{h}$ witnesses the large nor liminf of $p$, then $A \backslash\{j\}$ for $\mathbf{u}(h)=(h, j)$ witnesses that the nor liminf $_{\text {of }} q$ decreases only slightly.)

So $q$ is constructed by strengthening each $x(h)$ in this way. Clearly $q \leq p$ is still a valid condition, and forces $\underset{\sim}{r} \in \underset{\sim}{M}$, as $\underset{\sim}{r} \uparrow I_{\mathrm{nm},(h, \mathbf{u}(h))}$ disagrees with $\underset{\sim}{y_{\alpha}}$ for all $h \geq \min \left(\mathrm{w}^{p}\right)$.

Corollary 7.3.2 $\mathbb{Q}$ forces $\operatorname{non}(\mathcal{M}) \geq \kappa_{\mathrm{nm}}$.

Proof Assume that $\kappa_{\mathrm{nm}}>\aleph_{1}$ (otherwise there is nothing to show). Fix a condition $p$ and $\kappa<\kappa_{\mathrm{nm}}$ and names $\left(r_{i}\right)_{i \in \kappa}$ of reals. It is enough to show that there is an $\alpha \in \mathbf{\Xi}_{\mathrm{nm}}$ such that $p$ forces that $\left\{\underset{\sim}{r_{i}}: i \in \kappa\right\}$ is a subset of the meager set $\underset{\sim}{M_{\alpha}}$.

For each $i$ fix a maximal antichain $A_{i}$ below $p$ such that each $\tilde{a} \in A_{i}$ rapidly reads $\underset{\sim}{r}$. Due to $\aleph_{2}$-cc, and since $\kappa_{\mathrm{nm}}>\aleph_{1}$ and $\kappa_{\mathrm{nm}}>\kappa$, we can find an index $\alpha \in \Xi_{\mathrm{nm}}$ not appearing in the support of any condition in any $A_{i}$. According to the previous lemma, every element $a \in A_{i}$ (and hence also $p$ itself) forces that $\underset{\sim}{r_{i}} \in \underset{\sim}{M_{\alpha}}$.

\section{The nn part}

\subsection{The subatomic creatures for type nn}

We describe the subatomic families $\mathrm{K}_{\mathrm{nn}, b}^{\prime}$, depending on a parameter $b$.

Definition 8.1.1 (1) Fix an interval $I$ large enough such that (4) is satisfied (and in particular $|I|>b$ ). As in the nm subatoms, we assume that this interval $I$ is disjoint to all intervals previously chosen.

(2) The basic set of all possibilities, POSS, consists of all subsets $X$ of $2^{I}$ with relative size $1-1 / 2^{b}$ :

$$
\operatorname{POSS}:=\left\{X \subseteq 2^{I}:|X|=\left(1-1 / 2^{b}\right)\left|2^{I}\right|\right\}
$$

(3) A subatom $C=\operatorname{poss}(C)$ is a subset of POSS, where we set

$$
\begin{gathered}
\operatorname{nor}(C):=\frac{1}{b} \log _{b}\left(\operatorname{nor}_{0}(C)\right), \text { where } \\
\operatorname{nor}_{0}(C):=\min \left\{|Y|: Y \subseteq 2^{I},(\forall X \in \operatorname{poss}(C)) X \cap Y \neq \emptyset\right\} .
\end{gathered}
$$

(4) We require nor(POSS) $>b$ [thus satisfying (6.1.2)].

(5) We set $H^{\prime}(\mathrm{nn},=b):=\max (I)+1$.

Note that nor 0 of the subatom with full possibility set is approximately $2^{|I|} / 2^{b}$. In particular, for large $I$ the norm gets large, i.e., we can satisfy (4).

Lemma 8.1.2 (1) The subatomic family has strong b-bigness (i.e., satisfies the requirement (6.1.1)). 
(2) Given $E \subseteq 2^{I}$ and a subatom $C$, then the subatom $C^{\prime}$ with possibilities $\{H \in$ $\operatorname{poss}(C): H \cap E=\emptyset\}$ satisfies $\operatorname{nor}_{0}\left(C^{\prime}\right) \geq \operatorname{nor}_{0}(C)-|E|$.

(3) From the above it follows that: If $|E| \leq b^{\text {nor }(C)} / 2$, then $\operatorname{nor}\left(C^{\prime}\right) \geq \operatorname{nor}(C)-$ $\log _{b}(2)$.

Proof (1): Fix $F: \operatorname{poss}(C) \rightarrow b$. Let $C_{i}$ be the subatom with $F\left\lceil\operatorname{poss}\left(C_{i}\right)=i\right.$ for all $i \in b$. Assume that all $C_{i}$ have nor 0 at most $r$, witnesses by $X_{i} \subseteq 2^{I}$. Then $\bigcup X_{i}$ witnesses that $\operatorname{nor}_{0}(C) \leq b \cdot r$. So $\operatorname{nor}(C) \leq \log _{b}(b \cdot r) / b \leq 1 / b+\max \left(\operatorname{nor}\left(C_{i}\right)\right)$. So there is at least one $i$ with $\operatorname{nor}\left(C_{i}\right) \geq \operatorname{nor}(C)-1 / b$, as required.

(2): Assume $Y$ witnesses nor $0\left(C^{\prime}\right)$, then $Y \cup E$ witnesses nor ${ }_{0}(C)$.

(3):

$$
\begin{aligned}
\frac{b^{\text {nor }(C)}}{2} & =\frac{\operatorname{nor}_{0}(C)^{1 / b}}{2}=\left(\frac{\operatorname{nor}_{0}(C)}{2^{b}}\right)^{1 / b} \\
& \leq\left[\left(1-\frac{1}{2^{b}}\right)^{1 / b} \cdot \operatorname{nor}_{0}(C)^{b}\right]^{1 / b}=\left(1-\frac{1}{2^{b}}\right) \cdot \operatorname{nor}_{0}(C)
\end{aligned}
$$

\subsection{The generic object}

The following paragraph is just as in the nm case 7.2:

According to Sect. 6.1, when constructing the forcing at subatomic sublevels $\mathbf{u}$, we use for all $\xi \in \Xi_{\mathrm{nn}}$ the subatomic family $\mathrm{K}_{\xi, \mathbf{u}}=\mathrm{K}_{\mathrm{nn}, b(\mathbf{u})}^{\prime}$ living on some interval $I$, which we temporarily call $I_{\mathrm{nn}, \mathbf{u}}$. Also, if $p$ rapidly reads $\underset{\sim}{r}$, then $\underset{\sim}{r} \uparrow I_{\mathrm{nn}, \mathbf{u}}$ is decided below $\leq \mathbf{u}$.

Fix $\alpha$ of type nn. Recall that the generic object $y_{\alpha}$ assigns to each subatomic sublevel $\mathbf{u}$ the element $\underset{\sim}{R} R_{\alpha, \mathbf{u}}$ of $\operatorname{POSS}_{\alpha, \mathbf{u}}$ chosen by the generic filter. So $\underset{\sim}{R}, \mathbf{u}$ is a subset of $2^{I_{\mathrm{nn}, \mathbf{u}}}$ of relative size $\left(1-1 / 2^{b(\mathbf{u})}\right)$.

Note that $b(\mathbf{u})$ is strictly monotone [cf. (6.1.3)], and hence $\prod_{\mathbf{u} \text { subatomic sublevel }}(1-$ $\left.1 / 2^{b(\mathbf{u})}\right)>0$. Therefore

$$
\left\{x \in 2^{\omega}: \forall \mathbf{u}: x \uparrow I_{\mathrm{nn}, \mathbf{u}} \in \underset{\sim}{R} \boldsymbol{\sim}_{\alpha, \mathbf{u}}\right\}
$$

is positive, and

$$
\left\{x \in 2^{\omega}: \forall^{\infty} \mathbf{u}: x \uparrow I_{\mathrm{nn}, \mathbf{u}} \in \underset{\sim}{R} R_{\alpha, \mathbf{u}}\right\}
$$

has measure one. Therefore

$$
\underset{\sim}{N} N_{\alpha}:=\left\{x \in 2^{\omega}: \exists^{\infty} \mathbf{u}: x \uparrow I_{\mathrm{nn}, \mathbf{u}} \notin \underset{\sim}{R} R_{\alpha, \mathbf{u}}\right\}
$$

is a null set. (Here, $\mathbf{u}$ ranges over all subatomic sublevels.) 


\section{$8.3 \operatorname{non}(\mathcal{N}) \geq \kappa_{\mathrm{nn}}$}

Lemma 8.3.1 Let $p \in \mathbb{Q}$ rapidly read $\underset{\sim}{r} \in 2^{\omega}$ not using $\alpha \in \Xi_{\mathrm{nn}}$. Then $p$ forces $r \in N_{\alpha}$.

Proof As in 7.3.1, it is enough to find a $q \leq p$ forcing $r \in N_{\alpha}$; and we assume that $p$ is pruned and that $\alpha \in \operatorname{supp}(p)$.

We construct $q$ purely stronger than $p$ by induction, only modifying subatoms at index $\alpha$ (and decreasing their subatom norms by at most 1 ):

Pick a subatomic sublevel $\mathbf{u}$ (higher than any sublevel previously considered) where $\alpha$ is active with the subatom $C$ "living" on $I:=I_{\mathrm{nn}, \mathbf{u}}$.

$\underset{r}{\uparrow} I$ is decided $\leq \mathbf{u}$ and therefore even below $\mathbf{u}$ (as $\underset{\sim}{r}$ is read from $p$ not using $\alpha$; and due to modesty $\alpha$ is the only index active at sublevel $\mathbf{u})$. So the set $E$ of possibilities for $\underset{\sim}{r} I$ has size at most maxposs $(<\mathbf{u})$, and we can remove them all from the subatom at $C$ while decreasing the norm by at most 1, according to Lemma 8.1.2(2) and (6.1.4).

Repeat this for infinitely many sublevels $\mathbf{u}$.

Just as in 7.3.2, this implies:

Corollary 8.3.2 $\mathbb{Q}$ forces $\operatorname{non}(\mathcal{N}) \geq \kappa_{\mathrm{nn}}$.

\section{Some simple facts about counting}

We now list some simple combinatorial properties that will be used for the definitions and proofs in the cn-part.

\subsection{Large families of positive sets have positive intersection, nor ${ }^{\text {ก }}$}

Lemma 9.1.1 For $\delta \in(0,1)$ and $\ell \in \omega$ there are $M(\delta, \ell) \in \omega$ and $\varepsilon^{\cap}(\delta, \ell)>0$ such that: Whenever we have a probability space $\Omega$ and a family $\left(A_{i}: i<M\right)$ of sets of measure $\geq \delta$, we can find a subfamily of $\ell$ many sets whose intersection has measure at least $\varepsilon^{\cap}(\delta, \ell)$.

Proof By straightforward counting. ${ }^{34}$

We write $\chi_{B}$ for the characteristic function of $B$. Assume we have $M$ many sets $A_{i}$, and set $X \subset \Omega$ to contain all points that lie in at least $\ell$ many of the $A_{i}$. Then

$$
\delta \cdot M \leq \int \sum_{i \in M} \chi_{A_{i}} \leq \mu(X) \cdot M+\mu(\Omega \backslash X) \cdot(\ell-1) \leq \mu(X) \cdot M+\ell,
$$

and $\mu(X) \geq \delta-\ell / M$. So if we set

$$
M>2 \frac{\ell}{\delta},
$$

\footnotetext{
34 Originally we used a stronger statement for which we only had a more complicated proof. We are grateful to William B. Johnson for pointing out in http://mathoverflow.net/q/108380 that the statement in the current form has the obvious straightforward proof.
} 
then there are at least $\delta / 2$ many points in $X$. We can assign to each point $x \in X$ a subset $M_{x}$ of $M$ (of size at least $\ell$ ) by

$$
i \in M_{x} \text { iff } x \in A_{i} .
$$

This partitions $X$ into at most $2^{M}$ many sets; and at least one of the pieces has to have size at least

$$
\varepsilon^{\cap}(\delta, \ell):=\frac{\delta}{2 \cdot 2^{M}}
$$

Let us set $F_{b}^{0}:=1$ and $F_{b}^{n+1}=M\left(1 / b, F_{b}^{n}\right)$. We can use this notion to define a norm on natural numbers:

Definition 9.1.2 For $m>0$ : $\operatorname{nor}_{b}^{\cap}(m) \geq n$ iff $m \geq F_{b}^{n}$.

So we get the following:

Fix a measure space $\Omega$ and a sequence $\left(T_{i}\right)_{i \in A}$ of sets of measure $\geq$ $1 / b$. Then there is a subset $B \subseteq A$ such that $\operatorname{nor}_{b}^{\cap}(|B|) \geq \operatorname{nor}_{b}^{\cap}(|A|)-1$ and $\bigcap_{i \in B} T_{i}$ has measure $\geq \varepsilon^{\cap}(1 / b,|A|)$.

Note that without loss of generality the function $\varepsilon^{\cap}$ satisfies: $\varepsilon^{\cap}\left(\delta, \ell_{1}\right) \geq \varepsilon^{\cap}\left(\delta, \ell_{2}\right)$ whenever $\ell_{2}>\ell_{1}>0$. We write down the following trivial consequence of (9.1.3) for later reference:

Assume that $A$ is a subset of some finite set POSS. Fix a measure space $\Omega$ and a sequence $\left(T_{i}\right)_{i \in A}$ of sets of measure $\geq 1 / b$. Then there is a subset $B \subseteq A$ such that $\operatorname{nor}_{b}^{\cap}(|B|) \geq \operatorname{nor}_{b}^{\cap}(|A|)-1$ and $\bigcap_{i \in B} T_{i}$ has measure $\geq \varepsilon^{\cap}(1 / b,|\operatorname{POSS}|)$.

\subsection{Most large subsets do not cover a half-sized set}

Let $\Omega$ be the set of subsets of some finite set $A \in \omega$ of relative size $1-\epsilon$ (for $0<\epsilon<1 / 4$ ). (Since $A \in \omega$, we can write $A$ for the cardinality $|A|$.) I.e.: $x \in \Omega$ implies $x \subseteq A$ and $|x|=A \cdot(1-\epsilon)$. We can assume $A \gg 1 / \epsilon$ and that $A \cdot \epsilon$ is an integer.

Let $T \subseteq A$ be of relative size $\geq 1 / 2$, i.e., $|T| \geq A / 2$. Let $\Omega_{T}$ be the elements of $\Omega$ that cover $T$, i.e., $x \in \Omega^{T}$ iff $x \in \Omega$ and $T \subseteq x$.

We will use the following easy fact from combinatorics:

Fact 9.2.1 For any natural number $k \geq 2$, the quotient

$$
\frac{\left(\begin{array}{c}
2 N k \\
N
\end{array}\right)}{\left(\begin{array}{c}
N k \\
N
\end{array}\right)}
$$

tends to infinity with $N \rightarrow \infty$. 
Proof This can be checked with Stirling's approximation formula, or with the following elementary estimate: From

$$
\forall a, b: \frac{(a-b)^{b}}{b !} \leq\left(\begin{array}{l}
a \\
b
\end{array}\right) \leq \frac{a^{b}}{b !}
$$

we get

$$
N ! \cdot\left(\begin{array}{c}
2 N k \\
N
\end{array}\right) \geq(2 N k-N)^{N} \text { and } N ! \cdot\left(\begin{array}{c}
N k \\
N
\end{array}\right) \leq(N k)^{N}
$$

and hence

$$
\frac{\left(\begin{array}{c}
2 N k \\
N
\end{array}\right)}{\left(\begin{array}{c}
N k \\
N
\end{array}\right)} \geq \frac{(2 N k-N)^{N}}{(N k)^{N}} \geq\left(2-\frac{1}{k}\right)^{N} \rightarrow \infty .
$$

Lemma 9.2.2 Fix $b>2$ and a finite set $I$ with $|I|>b$. Let POSS be the family of subsets of $2^{I}$ of relative size $1-1 / 2^{b}$. For $m \in \omega$ we define nor $\dot{\bar{I}, b}(m):=\left\lfloor m /\left(\begin{array}{c}|I| I \mid-1 \\ 2^{|I|-b}\end{array}\right)\right\rfloor$.

Then:

(1) For any $T \subseteq 2^{I}$ of at least relative size $1 / 2$ and for any $C \subseteq$ POSS there is a subset $D \subseteq C$ with nor $\dot{\bar{I}, b}(|D|) \geq$ nor $\dot{\bar{I}, b}(|C|)-1$ and $T \nsubseteq x$ for all $x \in D$.

(2) If I is chosen sufficiently large (with respect to $b$ ), then nor $\dot{\overline{\dot{I}}, b}$ (POSS) is large.

Proof (1) It is enough to show this in case $T$ has exactly size $2^{|I|-1}$. If $x \in C \backslash D$, then the set $2^{I} \backslash x$ has size $2^{|I|-b}$ and is a subset of $2^{I} \backslash T$. So there are at most $\left(\begin{array}{c}2^{|I|-1} \\ 2^{|I|-b}\end{array}\right)$ possibilities for $2^{I} \backslash x$, hence (by definition of nor $\dot{\bar{I}, b}$ ) we get nor $\dot{\div}(C \backslash D) \leq 1$. From the implication

$$
x \leq y \text { and }\lfloor x-y\rfloor \leq 1 \Rightarrow\lfloor x\rfloor-\lfloor y\rfloor \leq 1
$$

we get nor $\dot{\bar{I}, b}(C)-$ nor $\dot{\bar{I}, b}(D) \leq 1$.

(2) Note that the cardinality of POSS is equal to $\left(2_{2^{|I|-b}}^{2^{|I|}}\right.$. Using Fact 9.2.1 with $N:=2^{|I|-b}$ and $k:=2^{b-1}$ we get that $\left(2_{2|I|-b}^{2|I|}\right) /\left(2_{2}^{|I|-1 \mid-b}\right)$ is large for large $I$.

\subsection{Providing bigness}

In this section, we write $\log$ to denote $\log _{2}$.

Apart from unimportant rounding effects, $\log$ of nor $\div$ satisfies 2-bigness (and the same for nor ${ }^{\cap}$ ). Instead of thinking about such effects, we just define for any norm a 2-big version. Actually, we define a 2-big version of the combinations of two norms (of course, any finite number of norms can be combined in this way): 
Definition 9.3.1 Assume that nor ${ }_{1}$, nor $2: \omega \rightarrow \omega$ are weakly increasing and converge to infinity.

Then we define lognor $=\operatorname{lognor}\left(\right.$ nor $_{1}$, nor 2$): \omega \rightarrow \omega$ as follows: By induction on $m$, we define $\operatorname{lognor}(x) \geq m$ by the conjunction of the following clauses:

- $\operatorname{nor}_{1}(x) \geq m$ and $\operatorname{nor}_{2}(x) \geq m$.

- $\operatorname{lognor}\left(\left\lfloor\frac{x}{2}\right\rfloor\right) \geq m-1$.

- If $y \in \omega$ and $i \in\{1,2\}$ satisfies $\operatorname{nor}_{i}(y) \geq \operatorname{nor}_{i}(x)-1$, then $\operatorname{lognor}(y) \geq m-1$.

We set lognor $(x):=\operatorname{lognor}\left(\right.$ nor $^{\bigcap}$, nor $\div$ ).

Lemma 9.3.2 Let lognor $=\operatorname{lognor}\left(\right.$ nor $_{1}$, nor $\left._{2}\right)$.

- $\operatorname{lognor}(x)$ is a well-defined natural number for all $x$, i.e., there is a maximal $m$ such that $\operatorname{lognor}(x) \geq m$ holds.

- lognor is weakly increasing and diverges to infinity.

- lognor has 2-bigness: If $F: m \rightarrow 2$ is a coloring function and $\operatorname{lognor}(m)=n$, then there is some $c \in 2$ such that $\operatorname{lognor}\left(F^{-1}(c)\right) \geq n-1$.

- So if we define $\operatorname{nor}_{b}(x)$ as $\frac{\operatorname{lognor}(x)}{[\log (b)\rceil}$, then $\operatorname{nor}_{b}$ will be b-big.

- If $\operatorname{nor}_{i}(y) \geq \operatorname{nor}_{i}(x)-1$ for some $i \in\{1,2\}$, then $\operatorname{lognor}(y) \geq \operatorname{lognor}(x)-1$.

Proof "Well-defined" follows from $\operatorname{lognor}(x) \leq \operatorname{nor}_{i}(x)$.

Monotonicity follows from the monotonicity of nor 1 and nor 2 .

We now prove that by induction on $m$ that there are only finitely many $x$ with $\operatorname{lognor}(x)<m$. For $m=0$ this is obvious, as all $x$ satisfy $\operatorname{lognor}(x) \geq 0$. For $m>0$ : $\operatorname{lognor}(x)<m$ iff either $\operatorname{nor}_{1}(x)<m$ or $\operatorname{nor}_{2}(x)<m$ or lognor $\left(\left\lfloor\frac{x}{2}\right\rfloor\right)<m-1$ or there is some $y$ and some $i \in\{1,2\}$ with $^{n o r}(y) \geq \operatorname{nor}_{i}(x)-1$ and $\operatorname{lognor}(y)<m-1$; for each case there are only finitely many possibilities.

2-bigness and the last item follow directly from the definition. $b$-bigness is Lemma 2.1.7.

\section{The en part}

\subsection{The subatomic creatures for type cn}

We now describe the subatomic families $\mathrm{K}_{\mathrm{cn}, b}^{\prime}$ used for the cn-indices.

Definition 10.1.1 (1) Fix an interval $I$ which is large enough to satisfy (4). In particular, $|I|>b$. Again, we assume that this interval is disjoint to all intervals previously chosen.

(2) The basic set of all possibilities and the set of subatoms is the same as in the nn-case 8.1.1 (but the norm will be different). So POSS consists of all subsets $X$ of $2^{I}$ with relative size $1-1 / 2^{b}$ :

$$
\operatorname{POSS}=\left\{X \subseteq 2^{I}:|X|=\left(1-1 / 2^{b}\right)\left|2^{I}\right|\right\}
$$

(3) A subatom $C$ is a subset of POSS, with $\operatorname{poss}(C):=C$, and

$$
\operatorname{nor}(C):=\frac{\operatorname{lognor}\left(\operatorname{nor}_{b}^{\bigcap}, \text { nor }_{i, b}^{\dot{\bar{i}}}\right)(|C|)}{2^{\min (I)} \cdot b^{2}}
$$


(4) We require nor(POSS) $>b$ [thus satisfying (6.1.2)].

(5) We set $H^{\prime}(\mathrm{cn},=b):=\max \left(H_{0}^{\prime}, H_{1}^{\prime}\right)$ for $H_{0}^{\prime}:=2^{\left(2^{|| \mid-b}\right)}$ and $H_{1}^{\prime||}:=$ $1 / \varepsilon^{\cap}(1 / b,|\operatorname{POSS}|)$, where $\varepsilon^{\cap}$ is defined in 9.1.1.

Note that $H^{\prime}(\mathrm{cn},=b)>\left|\mathrm{K}_{\mathrm{cn}, b}^{\prime}\right|$ (this is what we need $H_{0}^{\prime}$ for).

Recall that lognor satisfies 2-bigness, so after dividing by $b$ (actually, $\left\lceil\log _{2}(b)\right\rceil \cdot b$ would be sufficient) we get strong $b$-bigness [i.e., the norm satisfies the requirement (6.1.1)].

Note that (in contrast to the $\mathrm{nn}$ case) this norm is a counting norm, i.e., nor $(C)$ only depends on $|C|$, not on the "structure" of $C$.

\subsection{The generic object}

Just as in the nn-case, we set $I_{\mathrm{nn}, \mathbf{u}}$ to be the $I$ used for $\mathrm{K}_{\mathrm{nn}, b(\mathbf{u})}^{\prime}$; and we define $\underset{\sim}{N_{\alpha}}$ analogously to the nn-case. ${ }^{35}$

As before, $\underset{\sim}{N}$ is a name for a null set, and a real $r$ is in ${\underset{\sim}{N}}_{\alpha}$ iff there are infinitely many sublevels $\mathbf{u}$ such that $r \uparrow I_{\mathrm{Cn}, \mathbf{u}}$ is not in the possibility $X$ of $\mathrm{K}_{\mathrm{Cn}, \mathbf{u}}^{\prime}=\mathrm{K}_{\alpha, \mathbf{u}}$ that is chosen by the generic filter.

This time, the purpose of $N_{\alpha}$ is not to cover all reals not depending on $\alpha$, but rather to avoid being covered by any null set not depending on $\alpha$.

Lemma 10.2.1 Fix a subatomic sublevel $\mathbf{u}$, an index $\alpha \in \Xi_{\mathrm{Cn}}$ and a subatom $C \in$ $K_{\mathrm{cn}, \mathbf{u}}=K_{\alpha, \mathbf{u}}$.

(1) Given $T \subseteq 2^{I_{\mathrm{cn}, \mathrm{u}}}$ of relative size $\geq 1 / 2$ we can strengthen $C$ to $D$, decreasing the norm by at most $1 / 2^{\min (I)} \cdot b(\mathbf{u})$ such that $T \nsubseteq X$ for all $X \in \operatorname{POSS}(D)$.

(2) Fix a probability space $\Omega$ and a function $F$ that maps every $X \in \operatorname{poss}(C)$ to $F(X) \subseteq \Omega$ of measure $\geq 1 / b(\mathbf{u})$. Then we can strengthen $C$ to $D$, decreasing the norm by at most $1 / 2^{\min I} \cdot b(\mathbf{u})$ such that $\bigcap_{X \in \operatorname{poss}(D)} F(X)$ has measure at least $1 / b(\mathbf{u}+1)$. Here, $\mathbf{u}+1$ denotes the smallest subatomic sublevel above $\mathbf{u}$.

Proof This is an immediate consequences of (9.1.4), 9.2.2 and 9.3.2, just note that

$$
b(\mathbf{u}+1)>H^{\prime}(\mathrm{cn},=b(\mathbf{u})) \geq 1 / \varepsilon^{\cap}(1 / b(\mathbf{u}),|\operatorname{POSS}|) .
$$

Again, let $\mathbf{u}+1$ denote the smallest subatomic sublevel above $\mathbf{u}$. Then

$$
b(\mathbf{u}+1)>H^{\prime}(\mathrm{cn},=b(\mathbf{u}))>\left|\mathrm{K}_{\mathrm{cn}, b(\mathbf{u})}\right| .
$$

In other words,

The cardinality of $\mathrm{K}_{\mathrm{cn}, b(\mathbf{u})}$ is less than $b(\mathbf{u}+1)$.

\footnotetext{
$\overline{35}$ Of course, generally $I_{\mathrm{Cn}, \mathbf{u}} \neq I_{\mathrm{nn}, \mathbf{u}}$, so $\underset{\sim}{N}$ for $\alpha \in \Xi_{\mathrm{nn}}$ lives on a different domain than ${\underset{\sim}{\beta}}_{\beta}$ for $\beta \in \Xi_{\mathrm{Cn}}$.
} 


\subsection{Names for null sets}

Let $T \subseteq 2^{<\omega}$ be a tree (without terminal nodes) of measure 1/2. (Such trees correspond bijectively to closed sets of measure $1 / 2$.) Then the set

$$
N_{T}:=2^{\omega} \backslash \bigcup\{r+[T]: r \in \mathbb{Q}\} .
$$

is a null set (closed under rational translations). Conversely, for every null set $N$ there is such a $T$ with $N \subseteq N_{T}$.

The relative measure of $s$ in $T$ (for $s \in 2^{n}, n \in \omega$ ) is defined as $\mu([T] \cap[s]) \cdot 2^{n}$. For completeness, we say that the relative measure of $s$ is 0 if $s \notin T$. (Analogously, we can define the relative measure of a node $s$ in a finite tree $T \subseteq 2^{\leq m}$ with no terminal nodes of height $<m$.) Note the following easy consequence of the Lebesgue density theorem:

Fact 10.3.2 If $T$ is a tree without terminal nodes, $s \in T$ has positive relative measure, and $\delta<1$, then there is a $t>s$ with relative measure $>\delta$. (And for all levels above the level of $t$, there is an extension $t^{\prime}>t$ which also has relative measure $>\delta$.)

By removing nodes with relative measure 0 , the measure of $T$ does not change. We give such trees a name:

Definition 10.3.3 $T$ is a pruned- $1 / 2$ tree, if $T \subseteq 2^{<\omega}$ has measure $1 / 2$ and has no nodes of relative measure zero (and in particular no terminal nodes).

Note that each null set is contained in $N_{T}$ for some pruned-1/2 $T$. So instead of investigating arbitrary names for null sets, we will consider names $\underset{\sim}{T}$ for pruned-1/2 trees.

Note that there are fewer than $2^{2^{h}}$ many possibilities for the level $h$ of $\underset{\sim}{T}$. So we can "code" $\underset{\sim}{T}$ by a real $\underset{\sim}{\underset{\sim}{r}} \in 2^{\omega}$ such that $\underset{\sim}{T} \uparrow h$ is determined by $\underset{\sim}{r}\left\lceil 2^{2^{(h+1)}}\right.$.

Assume that $p$ rapidly reads this $\underset{\sim}{r}$. Then $\underset{\sim}{T} \uparrow\left(\max \left(I_{\mathrm{Cn}, \mathbf{u}}\right)+\tilde{1}\right)$ is determined $\leq \mathbf{u}$ [according to (6.1.6) and 10.1.1(5)].

We will describe this situation by " $p$ rapidly reads $T$ ".

\section{$10.4 \operatorname{cof}(\mathcal{N}) \geq \kappa_{\mathrm{cn}}$}

Lemma 10.4.1 Let $p \in Q$ rapidly read the pruned-1/2 tree $\underset{\sim}{T}$ not using the index

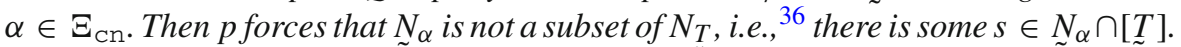

Proof We can assume that $p$ is pruned and that $\alpha \in \operatorname{supp}(p)$. It is enough to find a name $\underset{\sim}{r} \in 2^{\omega}$ and a $q \leq p$ forcing $\underset{\sim}{r} \in \underset{\sim}{N} N_{\alpha} \cap[T]$. For this, we will inductively modify $p$ at infinitely many sublevels $\mathbf{u}$ (resulting in the 1-purely stronger $q$ ):

Let $\mathbf{u}$ be a subatomic sublevel (above all the sublevels that we have already modified), where $\alpha$ is the active index with subatom $C$ of norm at least 10, living on the interval $I:=I_{\mathrm{Cn}, \mathbf{u}}$.

\footnotetext{
36 as $N_{\alpha}$ is closed under rational translates.
} 
The finite tree $\underset{\sim}{T^{\prime}}:=\underset{\sim}{T} \uparrow \max (I)+1$ is determined $\leq \mathbf{u}$, and even $<\mathbf{u}$, as $\underset{\sim}{T}$ does not depend on $\alpha$ (as usual, note that due to modesty $\alpha$ is the only active index at sublevel $\mathbf{u})$. In particular the set $Y$ of potential values of $\underset{T^{\prime}}{\prime}$ has size $\leq \operatorname{maxposs}(<\mathbf{u})$.

We now enumerate all $T^{*} \in Y$ and $t \in T^{*} \cap 2^{\min (I)}$ with relative measure (in $T^{*}$ ) at least $1 / 2$. There are at most maxposs $(<\mathbf{u}) \times 2^{\min (I)}$ many such pairs $\left(T^{*}, t\right)$.

Starting with $C^{0}:=C$, we iteratively use Lemma 10.2.1(1) to strengthen the subatom $C^{n}$ to some $C^{n+1}$ such that for the current $\left(T^{*}, t\right)$ and all $X \in \operatorname{poss}\left(C^{n+1}\right)$ there is some $t^{\prime} \in 2^{I} \backslash X$ such that $t \frown t^{\prime} \in T^{*}$.

So in the end we get a subatom $D \leq C$ of norm $\geq \operatorname{nor}(C)-1$ such that for all $\left(T^{*}, t\right)$ and $X \in \operatorname{poss}(D)$ there is some $t^{\prime} \in 2^{I} \backslash X$ with $t \frown t^{\prime} \in T^{*}$.

In this way, we modify infinitely many sublevels $\mathbf{u}$, resulting in a condition $q \leq p$.

Now work in the forcing extension, where $q$ is in the generic filter. We can now construct by induction an element $r$ of $\underset{\sim}{N} \cap \cap[\underset{\sim}{T}]$ (i.e., $r\left\lceil I_{\mathrm{Cn}, \mathbf{u}}\right.$ is not in the generically chosen $X$ at index $\alpha$ and sublevel $\mathbf{u}$, for infinitely many sublevels $\mathbf{u}$.)

Assume we already have $r\lceil n \in T$ for some $n$. Since $T$ has no nodes of relative norm 0 , there is a $h^{\prime}>n$ and an $t^{\prime} \in \tilde{T} \cap 2^{h^{\prime}}$ extending $\tilde{r} \uparrow n$ with relative measure $\geq 1 / 2$ (see 10.3.2). Pick a sublevel u such that: $\min (I)=: h>h^{\prime}$ for $I:=I_{\mathrm{Cn}, \mathbf{u}}$, and $\mathbf{u}$ was considered in our construction of $q$. There is still some $t \in 2^{h^{\prime}}$ extending $\underset{\sim}{r}\left\lceil n\right.$ of relative measure $1 / 2$. Set $T^{*}:=\underset{\sim}{T} \uparrow \max (I)+1$. Note that in our construction of $q$, when considering $\mathbf{u}$, we dealt with the pair $\left(T^{*}, t\right)$, and thus made sure for all $X \in \operatorname{poss}(q(\alpha, \mathbf{u}))$ (so in particular for the one actually chosen by the generic filter) there is some $t^{\prime} \in 2^{I}$ such that $t \frown t^{\prime} \in T^{*}$ and $t^{\prime} \notin X$. So we can just set $r\left\lceil\max I:=t^{\frown} t^{\prime}\right.$.

Corollary 10.4.2 $\mathbb{Q}$ forces that $\operatorname{cof}(\mathcal{N}) \geq \kappa_{\mathrm{cn}}$.

Proof This is very similar to the proof of 7.3.2: Assume that there is a $\aleph_{1} \leq \kappa<\kappa_{\mathrm{cn}}$ and a $p$ forcing that $\left.\underset{\sim}{\left(N_{i}^{*}\right.}\right)_{i \in \kappa}$ is a basis of null sets. As described above, we can assume that each $\underset{\sim}{N} N_{i}^{*}=N_{T_{i}}$ for some pruned-1/2 tree $\underset{\sim}{T}$ of measure $1 / 2$. For each $i$, fix a maximal antichain $A_{i}$ below $p$ of conditions rapidly reading $T_{\sim}$. The set $X:=\bigcup_{i \in \kappa, q \in A_{i}} \operatorname{supp}(q)$ has size $\kappa$, so there is an $\alpha \in \Xi_{\mathrm{Cn}} \backslash X$. Each $a \in A_{i}$ rapidly reads $T_{i}$ not using $\alpha$. So by the preceding lemma, $\underset{\sim}{N_{\alpha}} \nsubseteq N_{T_{i}}$ is forced by $a$ (and therefore by $p$, as $A_{i}$ is predense below $p$ ).

\section{$10.5 \operatorname{non}(\mathcal{N}) \leq \kappa_{\mathrm{nn}}$}

We want to show that the set $X$ of reals that are added by (or more precisely: rapidly read from) the $\mathrm{nm}$ and $\mathrm{nn}$ parts (i.e., not depending on the $\mathrm{cn}$ and Sacks parts) is not null.

Let $\mathbb{Q} \Xi_{\text {non-sk }}$ be the set of conditions $p$ with $\operatorname{supp}(p) \cap \Xi_{\mathrm{sk}}=\varnothing$. Recall that according to Lemma 3.6.1, $\mathbb{Q} \Xi_{\text {non-sk }}$ is a complete subforcing of $\mathbb{Q}$ (and satisfies $\omega^{\omega_{-}}$ bounding, rapid reading, etc). We have seen in 6.3 that the quotient of $\mathbb{Q}$ and $\mathbb{Q} \Xi_{\text {non-sk }}$ satisfies the Sacks property, and in particular that every null set $N$ in the $Q$-extension is contained in a null-set $N^{\prime} \supseteq N$ in the intermediate $\mathbb{Q}_{\Xi_{\text {non-sk }}}$-extension. 
So it is enough to show that $X$ is still non-null in the $\mathbb{Q} \Xi_{\text {non-sk }}$-extension; in other words, we can in the rest of the paper ignore the Sacks indices altogether (i.e., work in $\mathbb{Q} \Xi_{\text {non-sk }}$, or in other words assume that $\Xi_{\mathrm{sk}}=\emptyset$ ).

We have seen that the sets of the form $N_{T}$ for pruned-1/2 trees $T$ form a basis of null sets; so we just have to show the following:

Lemma 10.5.1 Let ${\underset{\sim}{*}}^{*}$ be a pruned-1/2 tree rapidly read by $p$. Then there is a $q \leq p$

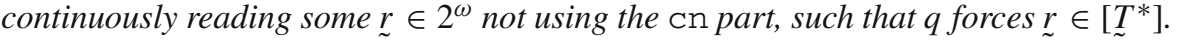
(As described above, the Sacks part is not used at all.)

As $\underset{\sim}{r} \in\left[{\underset{\sim}{T}}^{*}\right]$ implies $\underset{\sim}{r} \notin N_{T^{*}}$, and $\underset{\sim}{r}$ only depends on the nm and nn parts, we get:

Corollary 10.5.2 $\mathbb{Q}$ forces $\operatorname{non}(\mathcal{N}) \leq \kappa_{\mathrm{nn}}$.

To prove Lemma 10.5.1 we will use:

Lemma 10.5.3 Let $T$ be a tree of positive measure and fix $\epsilon>0$. Then for all sufficiently large $m \in \omega$ there are many fat nodes in $T \cap 2^{m}$, by which we mean:

$$
\mu\left(\left[T^{[s]}\right]\right) \geq 2^{-m}(1-\epsilon) \text { for at least }\left|[T] \cap 2^{m}\right| \cdot(1-\epsilon) \text { many } s \in T \cap 2^{m} .
$$

Proof Write $\mu$ for the measure of $[T]$. Note that $\left|T \cap 2^{m}\right| \cdot 2^{-m}$ decreases and converges to $\mu$. Hence from some $m$ on, we have

$$
\left|T \cap 2^{m}\right| \cdot 2^{-m}-\mu \epsilon^{2} \leq \mu .
$$

Let $l$ be the number of fat nodes at level $m$, and $s=\left|T \cap 2^{m}\right|-l$ the number of non-fat nodes. We want to show $l \geq 2^{m} \mu \cdot(1-\epsilon)$.

Clearly,

$$
\mu<l \cdot 2^{-m}+s \cdot 2^{-m}(1-\epsilon)=\left|T \cap 2^{m}\right| \cdot 2^{-m}-2^{-m} s \epsilon
$$

Combining (10.5.4) and (10.5.5), we get $\left|T \cap 2^{m}\right| \cdot 2^{-m}-\mu \epsilon^{2} \leq\left|T \cap 2^{m}\right| \cdot 2^{-m}-$ $2^{-m} s \epsilon$, and hence $s \leq 2^{m} \mu \cdot \epsilon$. As $l+s=\left|T \cap 2^{m}\right| \geq 2^{m} \mu$, we get $l \geq 2^{m} \mu \cdot(1-\epsilon)$, as required.

Proof of Lemma 10.5.1 We can assume that $p$ is pruned. By induction on $n \in \omega$, we construct:

(a) $k_{n} \in \omega$.

(b) A condition $q_{n} \leq p$ with $k_{n} \in \mathrm{w}^{q_{n}}$ such that $\operatorname{nor}\left(q_{n}, k^{\prime}\right) \geq n+6$ for all $k^{\prime} \geq k_{n}$ in $\mathrm{w}^{q_{n}}$.

(c) We will additionally require: $q_{n+1} \leq q_{n} ; q_{n+1}$ is identical to $q_{n}$ below $k_{n}$, and has norms $\geq n$ between $k_{n}$ and $k_{n+1}$.

(Therefore there is a limit condition $q_{\omega}$ stronger than each $q_{n}$.)

(d) $i_{n} \in \omega$ and a name $\underset{\sim}{s_{n}}$ for an element of $\underset{\sim}{T}{ }^{*} \cap 2^{i_{n}}$ such that $q_{n}$ decides $\underset{\sim}{s_{n}}$ below $k_{n}$ not using any cn-indices. 
(e) We additionally require that $i_{n}$ is "not too large" with respect to $k_{n}$, more particularly:

$$
2^{i_{n}+2}<b\left(\left(k_{n}, 0\right)\right)
$$

$\left(\left(k_{n}, 0\right)\right.$ is the the smallest subatomic sublevel above $k_{n}$.) (As $b$ is strictly monotone, it suffices to have $k_{n}>2^{i_{n}+2}$.)

(f) We additionally require: $i_{n+1}>i_{n}$, and $s_{n+1}$ is forced (by $q_{n+1}$ ) to extend $s_{n}$.

So $q_{\infty}$ will force that the union of the $\underset{\sim}{S_{n}}$ will be the required branch through $\underset{\sim}{T^{*}}$, proving the Lemma.

(g) We will also construct a name $T_{n}$, which is (forced by $q_{n}$ to be) a subtree of ${\underset{\sim}{T}}^{*}$ with stem $\underline{\sim}_{n}$ and relative measure $>1 / 2$ (i.e., $\left.\mu\left(\left[T_{n}\right]\right)>1 / 2 \cdot 2^{-i_{n}}\right)$, which is read continuously by $q_{n}$ not using any cn-indices below $k_{n} \cdot{ }^{37}$

We set $i_{0}:=0,{\underset{\sim}{0}}_{0}:=\langle\rangle$ and $\underset{\sim}{T_{0}}={\underset{\sim}{T}}^{*}$. We choose $k_{0}$ such that the norms of the compound creatures in $p$ are $\geq \tilde{6}$ above $k_{0}$ and set $q_{0}$ to be $p$ where we increase the trunk to $k_{0}$. So $\underset{\sim}{T_{0}}$ does not depend on any cn-indices below $k_{0}$ (as below $k_{0}$ there is only trunk and thus a unique possibility).

So assume we already have the objects mentioned above for some $n$ (i.e., $k_{n}, q_{n}$, $i_{n}, S_{n}$ and $\underset{\sim}{T_{n}}$ ). For notational simplicity we refer to them without the subscript $n$, i.e., we set $k:=k_{n}$ etc. We will now construct the objects for $n+1$.

(1) We choose $k^{*}$ so large that for each $\xi \in \operatorname{supp}(q(k)) \cap \Xi_{1 \mathrm{~s}}$ there is an atom $q_{n}(\xi, \ell)$ of norm $>n+2$ for some $\ell$ between $k$ and $k^{*}$.

(2) It is forced that Lemma 10.5.3 holds for $\underset{\sim}{T}$ and for $\epsilon:=1 / \operatorname{maxposs}\left(<k^{*}\right) \cdot \operatorname{maxposs}(<k)$. So we get a name $\underset{\sim}{m}$ for a level where there are many fat nodes. Using Lemma 5.3.1, we strengthen $q$ to $q^{1}$, not changing anything below $k^{*}$ and keeping all norms $\geq n+4$, such that we can find (in $V$ ) some $m>i$ which is forced by $q^{1}$ to be $\geq \underset{\sim}{m}$. Note that Lemma 10.5 .3 is forced to hold for this $m \geq \underset{\sim}{m}$ as well, i.e., there is a name of a "large" set $\underset{\sim}{L} \subseteq 2^{m}$ of "fat" nodes.

This $m$ will be our $i_{n+1}$. So $i_{n+1}>i_{n}$ is satisfied.

(3) So can further strengthen $q^{1}$ to $q^{2}$ not changing anything below $k^{*}$ and keeping all norms $\geq n+2$ such that $\underset{\sim}{L} \subseteq 2^{m}$ is essentially decided, i.e., decided below some level $k^{* *}>k^{*}$. Since we already assumed that $\underset{\sim}{T}$ is read continuously, we can assume that $q^{2}$ also decides $\underset{\sim}{T} \cap 2^{m}$ below $k^{* *}$. Also, we can assume that all norms of compound creatures in $q^{2}$ above (including) $k^{* *}$ are $>n+7$, and that $k^{* *}>2^{m+2}$.

This $k^{* *}$ will be $k_{n+1}$. Note that this ensures item (e) for $n+1$.

(4) $\underset{\sim}{L}$ is forced to be a subset of $\underset{\sim}{T} \cap 2^{m}$ of relative size $\geq(1-\epsilon)$, and both $\underset{\sim}{L}$ and $\underset{\sim}{T} \cap 2^{m}$ are decided below $k^{* *}$. Also, $\underset{\sim}{T} \cap 2^{m}$ does not depend on the cn-part below $k$. Therefore, we can construct a name $\underset{\sim}{L^{\prime}} \subseteq \underset{\sim}{L}$ that also does not depend on such coordinates, and such that $\underset{\sim}{L^{\prime}} \subseteq \underset{\sim}{T} \cap 2^{m}$ has relative size $\geq(1-\epsilon \cdot \operatorname{maxposs}(<k)) \geq 1 / 2$.

\footnotetext{
37 I.e.: For all $\ell$ there is a $k$ and a function defined on $\operatorname{poss}\left(q_{n},<k\right)$ giving the value of $T_{n} \cap 2^{\ell}$ such that the value is the same for $\eta, \eta^{\prime} \in \operatorname{poss}\left(q_{n},<k\right)$ that differ only on the cn-part below $k_{n}$.
} 
Proof: Each $\eta \in \operatorname{poss}\left(q_{2}, k^{* *}\right)$ determines objects $L_{\eta} \subseteq S_{\eta}$ (where $q^{2} \wedge \eta$ forces " $L_{\eta}=\underset{\sim}{L}$ and $S_{\eta}=\underset{\sim}{T} \cap 2^{m}$ "). We call $\eta_{1}, \eta_{2}$ equivalent if they differ only on the cn-part below $k$ (which implies $S_{\eta_{1}}=S_{\eta_{2}}$ ). Clearly, each equivalence class has size at most maxposs $(<k)$. For an equivalence class $[\eta]$, we set $L_{[\eta]}^{\prime}:=$ $\bigcap_{\eta^{\prime} \in[\eta]} L_{\eta^{\prime}}$. So the map assigning $\eta$ to $L_{[\eta]}^{\prime}$ defines a name (not depending on the cn-part below $k$ ) of a subset of $S_{\eta}$ of relative size $\geq 1 / 2$.

Recall that $\underset{\sim}{T}$ is forced to have stem $s \in 2^{i}$ and measure $>1 / 2 \cdot 2^{-i}$, so the cardinality of $\underset{\sim}{T} \cap 2^{m}$ is forced to be $>2^{m-i-1}$, and thus the cardinality of ${\underset{\sim}{\prime}}^{\prime}$ is forced to be $>2^{m-i-1}(1 / 2)=2^{m-i-2}>2^{m} / b((k, 0))$, according to item (e).

To summarize:

- $\underset{\sim}{T} \cap 2^{m}$ and its subset $\underset{\sim}{L^{\prime}}$ are decided by $q^{2}$ below $k^{* *}$, not using the cn-part below $k$.

- We set $\Omega=2^{m}$. (As a finite set, it carries the uniform probability measure.) $L^{\prime}$ as subset of $\Omega$ is forced to have measure $>1 / b((k, 0))$.

- $\tilde{q}^{2}$ forces that each $s \in \underset{\sim}{L^{\prime}}$ satisfies $\mu\left(\left[T_{\sim}^{[s]}\right]\right) \geq 2^{-m}(1-\epsilon)$.

(5) Now we glue $q^{2}$ between $k$ and $k^{* *}$, and replace all lim-sup subatoms between $k^{*}$ and $k^{* *}$ with singletons (not changing the lim-inf subatoms, nor anything between $k$ and $k^{*}$ ), resulting in $q^{*}$ and the compound creature $\mathfrak{d}^{*}=q^{*}(k)$ (with $m^{\mathrm{dn}}\left(\mathfrak{d}^{*}\right)=k, m^{\text {up }}\left(\mathfrak{d}^{*}\right)=k^{* *}$ and $\left.\operatorname{supp}\left(\mathfrak{d}^{*}\right)=\operatorname{supp}(q, k)\right)$. So above $k^{* *}, q^{*}$ is identical to $q^{2}$, and below $k^{*}$ it is identical to $q$.

Note that nor $\left(\mathfrak{d}^{*}\right) \geq n+2$ : Gluing results in a norm at least the minimum of the norms of the glued creatures; and replacing lim-sup subatoms above $k^{*}$ with singletons does not drop the norm below $n+2$ as we made sure that there are large subatoms between $k$ and $k^{*}$.

We will in the following find a strengthening $\mathfrak{d}^{* *}$ of $\mathfrak{d}^{*}$ with $\operatorname{nor}\left(\mathfrak{d}^{* *}\right) \geq \operatorname{nor}\left(\mathfrak{d}^{*}\right)-$ $2 \geq n$ and we will set $q_{n+1}$ to be $q^{*}$ where we replace $\mathfrak{d}^{*}$ with $\mathfrak{d}^{* *}$. Then items (b) and (c) will be satisfied for $n+1$.

(6) Recall that $q^{*}$ decides both $\underset{\sim}{L^{\prime}}$ and $\underset{\sim}{T} \cap 2^{m}$ below $k^{* *}$, not using the cn-part below $k$. Note that $\operatorname{poss}\left(q^{*},<k^{* *}\right)$ is isomorphic to $X \times Y \times Z$, for

- $X:=\operatorname{poss}\left(q^{*},<k\right)=\operatorname{poss}(q,<k)$,

- $Y$ are the possibilities of $\mathfrak{d}^{*}$ between $k$ and $k^{*}$, and

- $Z$ are the possibilities of $\mathfrak{d}^{*}$ between $k^{*}$ and $k^{* *}$ (which we can restrict to the lim-inf part, as there are only singletons in the lim-sup-part).

(7) Fix a $v \in Z$. We will now perform an induction on the (subatomic) sublevels $\mathbf{u}$ between $k$ and $k^{*}$, starting with the lowest one, $(k, 0)$. We assume that we have arrived in this construction at sublevel $\mathbf{u}$ with the active subatom $C$, and that we already have constructed the following:

- The (final) subatoms for all sublevels $\mathbf{v}$ below $\mathbf{u}$ (and above $k$ ), with subatomnorm at most 2 smaller than the norms of the original subatoms (i.e., those in $\left.\mathfrak{d}^{*}\right)$.

- (Preliminary) subatoms for all sublevels $\mathbf{u}^{\prime}$ above (including) $\mathbf{u}$ (and below $k^{*}$ ), where the norm of the subatom at $\mathbf{u}^{\prime}$ has been reduced from the original one by at most $K / b\left(\mathbf{u}^{\prime}\right)$, where $K$ is the number of steps already performed in the current induction (i.e., $K$ is the number of subatomic sublevels between $k$ and $\mathbf{u}$ ). So our current $C$ is one of these "preliminary subatoms". 
- A function $F^{\mathbf{u}}$ that maps each possibility $\eta \in X \times Y$ to a subset $F^{\mathbf{u}}(\eta)$ of $2^{m}$; such that for all $\eta$

- $F^{\mathbf{u}}(\eta)$ is forced to be a subset of $L_{\sim}^{\prime}$ by the condition $q^{*}$ modulo the fixed $v \in Z$, modulo $\eta$ and modulo the already constructed subatoms (the final ones as well as the preliminary ones). ${ }^{38}$

- $F^{\mathbf{u}}(\eta) \subseteq 2^{m}$ is of relative size $\geq 1 / b(\mathbf{u})$.

- $F^{\mathbf{u}}(\eta)$ does not depend on any cn-indices below $\mathbf{u}$.

The first sublevel, $(k, 0)$, is clear: there are no sublevels below where we have to define final subatoms, the preliminary subatoms above are just the original ones, and $F^{(k, 0)}$ is just given by the name $L^{\prime}$.

Now we perform the inductive step. If our subatom $C$ is not of cn-type, we do nothing ${ }^{39}$ and go to the next step. So let us assume that the current (preliminary) $C$ is of cn-type.

Let $Y^{-}$be $Y$ restricted to the sublevels below $\mathbf{u}$, and $Y^{+}$to the ones above. Every $^{40} \eta \in X \times Y$ can be written as $\left(\eta^{-}, \eta^{\mathbf{u}}, \eta^{+}\right)$for $\eta^{-} \in X \times Y^{-}, \eta^{\mathbf{u}} \in$ $\operatorname{poss}(C)$ and $\eta^{+} \in Y^{+}$.

When we fix some $\eta^{-} \in X \times Y^{-}$and $\eta^{+} \in Y^{+}$, the function $F^{\mathbf{u}}$ reduces to a function $F^{\eta^{-}, \eta^{+}}$that maps poss $(C)$ to subsets of $2^{m}$ of relative size $\geq 1 / b(\mathbf{u})$. So we can use Lemma 10.2.1(2) and strengthen $C$ to $D\left(\eta^{-}, \eta^{+}\right)$decreasing the norm by at most $1 / b(\mathbf{u})$ such that

$$
F^{\prime}\left(\eta^{-}, \eta^{+}\right):=\bigcap_{\mu \in \operatorname{poss}\left(D\left(\eta^{-}, \eta^{+}\right)\right)} F^{\eta^{-}, \eta^{+}}(\mu)
$$

is a set of measure $\geq 1 / b(\mathbf{u}+1)$.

For fixed $\eta^{+} \in Y^{+}$, we can iterate this strengthening for all $\eta^{-} \in X \times Y^{-}$: From $D$ to some $\tilde{D}:=D\left(\eta^{-}, \eta^{+}\right)$, then from $\tilde{D}$ to $D\left(\eta^{\prime-}, \eta^{+}\right)$for the next $\eta^{\prime-}$, etc., resulting in a $D\left(\eta^{+}\right)$with norm reduced by at most $\operatorname{maxposs}(<\mathbf{u}) / b(\mathbf{u})<1$.

Note that there are less than $b(\mathbf{u}+1)$ many possibilities for $D\left(\eta^{+}\right)$, cf (10.2.2). Finally we can use bigness of the $Y^{+}$-part, as stated in Lemma 6.1.5, to find successor subatoms at all sublevels above $\mathbf{u}$, resulting in a new set of possibilities $\tilde{Y}^{+} \subseteq Y^{+}$such that for each $\eta^{+} \in \tilde{Y}^{+}$we get the same $D:=D\left(\eta^{+}\right)$. This $D$ will be the (final) subatom at our current level $\mathbf{u}$.

We can now define

$$
F^{\mathbf{u}+1}(\eta):=\bigcap_{\mu \in \operatorname{poss}(D)} F^{\mathbf{u}}\left(\eta^{-}, \mu, \eta^{+}\right) .
$$

As above, this is a set of measure $\geq 1 / b(\mathbf{u}+1)$, does not depend on the cn-part $\leq \mathbf{u}$, and it is forced (modulo $D$ ) to be a subset of $\underset{\sim}{L^{\prime}}$.

\footnotetext{
38 See (5.1.9) for a definition of "modulo". If $\eta$ is not a compatible with the currently constructed (final and preliminary) subatoms, then $F^{\mathbf{u}}(\eta)$ is irrelevant.

39 Slightly more formally: we make the current preliminary subatom final, and set $F^{\mathbf{u}+1}:=F^{\mathbf{u}}$.

40 We are concerned only about the $\eta$ still are compatible with the currently constructed preliminary/final subatoms.
} 
We have now chosen the new final subatom $D$, the new preliminary subatoms and $F^{\mathbf{u}+1}$ in a way that we can perform the next step of the iteration.

(8) We perform the whole inductive construction of (7) for every $v \in Z$ independently (i.e., we start at the original $\mathfrak{d}^{*}$ for each $v \in Z$ ).

So for every $v$ we get a different sequence $\bar{D}(v)$ of subatoms between $k$ and $k^{*}$. Using bigness (again as in Lemma 6.1.5), we can thin out the subatoms between $k^{*}$ and $k^{* *}$, resulting in $Z^{\prime} \subseteq Z$, such that for each $v \in Z^{\prime}$ we get the same sequence $\bar{D}(v)=: \bar{D}$ which finally defines the compound creature $\mathfrak{d}^{* *}$ stronger than $\mathfrak{d}^{*}$.

We set $q_{n+1}$ to be $q^{*}$ with $\mathfrak{d}^{*}$ strengthened to $\mathfrak{d}^{* *}$, and we set $i_{n+1}:=m$ and $k_{n+1}:=k^{* *}$.

(9) Now work modulo $q_{n+1}$. So the final function $F$ of the induction in (7) gives us a name for a subset $\underset{\sim}{L^{\prime \prime}} \subseteq \underset{\sim}{L} \subseteq 2^{m}$ of positive relative size (in $2^{m}$ ), and the name $L_{\sim}^{\prime \prime}$ does not depend on any $\mathrm{cn}$ indices: Not on any below $k$, since we started with the name $\underset{\sim}{L}$ ' which did not depend on such subatoms; not on any between $k$ and $k^{*}$, as we removed this dependence sublevel by sublevel during the induction; and not on any $\mathrm{cn}$ subatoms between $k^{*}$ and $k^{* *}$, as $\mathrm{cn}$ indices are of lim-sup type, and we have only singleton subatoms for the lim-sup part between $k^{*}$ and $k^{* *}$.

So we can pick a non-cn-name $s_{n+1}$ for an arbitrary (the leftmost, say) element of $\underset{\sim}{L}$.

(10) $\quad q_{n+1}$ forces that $s_{n+1}$ is in $\underset{\sim}{L}$, i.e., a "fat" node, more specifically: $\underset{\sim}{\prime}:={\underset{\sim}{n}}_{n}^{\left[s_{n+1}\right]}$ has a measure greater than $\frac{1-\epsilon}{2^{m}}$.

The tree $\underset{\sim}{T}$ is read continuously by $q_{n}$ and therefore also by $q_{n+1}$. In particular, for each $\ell>m$ the finite tree $\underset{\sim}{T^{\prime}} \cap 2^{\ell}$ is decided below some $\ell^{\prime}$. For $\eta \in$ $\operatorname{poss}\left(q_{n+1},<\ell^{\prime}\right)$ let $T^{\ell, \eta}$ be the according value of $T_{\sim}^{\prime} \cap 2^{\ell}$ (a subset of $2^{\ell}$ with at least $2^{\ell} \cdot \frac{1-\epsilon}{2^{m}}$ elements). We call $\eta$ and $\eta^{\prime}$ equivalent if they differ only on the cn part below $k^{* *}$. Each equivalence class has size $\leq \operatorname{maxposs}\left(<k^{*}\right)$, as there are only singleton values in the lim-sup part between $k^{*}$ and $k^{* *}$. We assign to each equivalence class $[\eta]$ the tree $T^{\ell,[\eta]}:=\bigcap_{\eta^{\prime} \in[\eta]} T^{\ell, \eta^{\prime}}$. Then $T^{\ell,[\eta]}$ has size at least $2^{\ell} \cdot \frac{1-\operatorname{maxposs}\left(<k^{*}\right) \cdot \epsilon}{2^{m}}$ (and of course does not depend on the cn-part below $\left.k^{* *}\right)$. So the family $T^{\ell,[\eta]}$ defines a continuous name for a tree ${\underset{\sim}{n+1}}_{n+1}$ not depending on the cn-part below $k^{* *}$ with root $s_{n+1}$ and measure $>1 / 2^{m+1}$, as required.

\section{Switching $\mathrm{nm}$ and $\mathrm{nn}$}

It turns out that the same proof can be used for the following variant of Theorem 6.2.1, where the order of $\kappa_{\mathrm{nm}}$ and $\kappa_{\mathrm{nn}}$ is reversed:

Theorem 11.0.6 Assume (in V) CH, $\kappa_{\mathrm{nn}} \leq \kappa_{\mathrm{nm}} \leq \kappa_{\mathrm{cn}} \leq \kappa_{\mathrm{sk}}$ and $\kappa_{t}^{\aleph_{0}}=\kappa_{t}$ for $t \in\{\mathrm{nm}, \mathrm{nn}, \mathrm{cn}, \mathrm{sk}\}$. Then there is a forcing $\mathbb{Q}$ which forces

(1) $\operatorname{cov}(\mathcal{N})=\mathfrak{d}=\aleph_{1}$,

(2) $\operatorname{non}(\mathcal{N})=\kappa_{\mathrm{nn}}$,

(3) $\operatorname{non}(\mathcal{M})=\operatorname{cof}(\mathcal{M})=\kappa_{\mathrm{nm}}$, 
(4) $\operatorname{cof}(\mathcal{N})=\kappa_{\mathrm{cn}}$,

(5) $2^{\aleph_{0}}=\kappa_{\mathrm{sk}}$.

\section{Moreover, $\mathbb{Q}$ preserves all cardinals and all cofinalities.}

Proof We now use the cn-norm for the nm part as well. (Recall 7.1.2: We can use any nm-norm, as long as bigness is satisfied.) The proofs above do not change, apart the one of $\operatorname{non}(\mathcal{N}) \leq \kappa_{\mathrm{nn}}$ : In the inductive construction, we only had to do something at the $\mathrm{cn}$-indices, and we could ignore the nm-indices (as there were only few). In the new version, we have to include the nm-indices as well. But this is no problem: We now do exactly the same at nm-indices as at cn-indices (which we can, as the nm-norm is the same as the cn-norm).

Acknowledgements Open access funding provided by Austrian Science Fund (FWF). We are grateful to Diego Mejía for pointing out several embarrassing oversights. We also thank the anonymous referee for pointing out additional errors, and making numerous helpful suggestions for improving the text.

Open Access This article is distributed under the terms of the Creative Commons Attribution 4.0 International License (http://creativecommons.org/licenses/by/4.0/), which permits unrestricted use, distribution, and reproduction in any medium, provided you give appropriate credit to the original author(s) and the source, provide a link to the Creative Commons license, and indicate if changes were made.

\section{References}

1. Bartoszyński, T.: Additivity of measure implies additivity of category. Trans. Am. Math. Soc. 281(1), 209-213 (1984)

2. Brendle, J., Fischer, V.: Mad families, splitting families and large continuum. J. Symb. Log. 76(1), 198-208 (2011)

3. Bartoszyński, T., Judah, H.: Set Theory. On the Structure of the Real Line. A K Peters Ltd., Wellesley (1995)

4. Bartoszyński, T., Judah, H., Shelah, S.: The Cichoń diagram. J. Symb. Log. 58(2), 401-423 (1993)

5. Brendle, J.: Larger cardinals in Cichoń's diagram. J. Symb. Log. 56(3), 795-810 (1991)

6. Blass, A., Shelah, S.: Ultrafilters with small generating sets. Israel J. Math. 65(3), 259-271 (1989)

7. Cichoń, J., Kamburelis, A., Pawlikowski, J.: On dense subsets of the measure algebra. Proc. Am. Math. Soc. 94(1), 142-146 (1985)

8. Judah, H., Shelah, S.: The Kunen-Miller chart (Lebesgue measure, the Baire property, Laver reals and preservation theorems for forcing). J. Symb. Log. 55(3), 909-927 (1990)

9. Kamburelis, A.: Iterations of Boolean algebras with measure. Arch. Math. Log. 29(1), 21-28 (1989)

10. Krawczyk, A.: Consistency of A(c) \& B(m) \& non-A(m). Unpublished notes (1983)

11. Kellner, J., Shelah, S.: Decisive creatures and large continuum. J. Symb. Log. 74(1), $73-104$ (2009)

12. Kellner, J., Shelah, S.: Creature forcing and large continuum: the joy of halving. Arch. Math. Log. 51(1-2), 49-70 (2012)

13. Mejía, D.A.: Matrix iterations and Cichon's diagram. Arch. Math. Log. 52(3-4), 261-278 (2013)

14. Miller, A.W.: Some properties of measure and category. Trans. Am. Math. Soc. 266(1), 93-114 (1981)

15. Miller, A.W.: Additivity of measure implies dominating reals. Proc. Am. Math. Soc. 91(1), 111-117 (1984)

16. Raisonnier, J., Stern, J.: Mesurabilité et propriété de Baire. C. R. Acad. Sci. Paris Sér. I Math. 296(7), 323-326 (1983)

17. Rosłanowski, A., Shelah, S.: Norms on possibilities. I. Forcing with trees and creatures. Mem. Am. Math. Soc. 141(671), xii+167 (1999)

18. Shelah, S.: Proper and Improper Forcing. Perspectives in Mathematical Logic, 2nd edn. Springer, Berlin (1998) 